\title{
SIMULATION OF LANDSCAPE DISTURBANCES AND THE EFFECT OF CLIMATIC CHANGE
}

\author{
Final Report
}

for Period July 15, 1990 - January 14, 1993

William L. Baker

\section{Department of Geography and Recreation University of Wyoming \\ Laramie, Wyoming 82071}

April 1993

Prepared for

THE U.S. DEPARTMENT OF ENERGY

AGREEMENT NO. DE-FG02-90ER60977 


\section{NOTICE}

This report was prepared as an account of work sponsored by the United States Government. Neither the United States nor the Department of Energy, nor any of their employees, nor any of their contractors, subcontractors, or their employees, makes any warranty, express or implied, or assumes any legal liability or responsibility for the accuracy, completeness, or usefulness of any information, apparatus, product, or process disclosed or represents that its use would not infringe privately-owned rights. 
TABLE OF CONTENTS

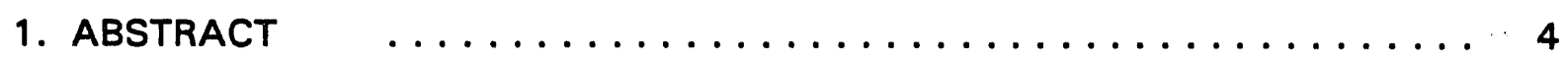

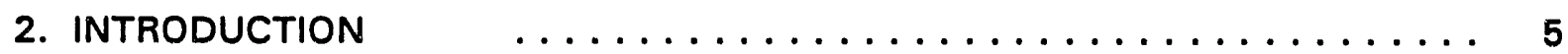

3. OVERVIEW OF RESEARCH COMPLETED $\ldots \ldots \ldots \ldots \ldots \ldots \ldots \ldots$

4. RESEARCH STILL IN PROGRESS $\ldots \ldots \ldots \ldots \ldots \ldots \ldots \ldots \ldots \ldots$

5. BIBLIOGRAPHY $\ldots \ldots \ldots \ldots \ldots \ldots \ldots \ldots \ldots \ldots \ldots \ldots$

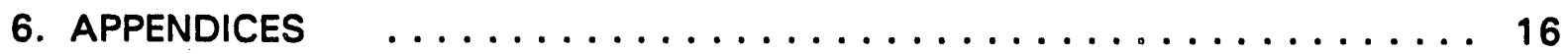

A. Reprints

Baker, W.L. 1992. The landscape ecology of large disturbances in the design and management of nature reserves. Landscape Ecology 7: 181-194.

Baker, W.L. 1993. Spatially heterogeneous multi-scale response of landscapes to fire suppression. Oikos 66: 66-71.

Baker, W.L. and Y. Cai. 1992. The r.le programs for multiscale analysis of landscape structure using the GRASS geographical information system. Landscape Ecology 7: 291-302.

B. Preprints

Baker, W.L. (in review). Spatial modeling of the restoration of landscape structure altered by fire suppression. Environmental Management, in review.

Baker, W.L. (in review). Longterm response of disturbance landscapes to human intervention and global change. Landscape Ecology, in review.

C. SIMSCRIPT code for the DISPATCH model

D. Documentation for the r.le software programs 


\section{ABSTRACT}

Altering the natural disturbance regime of a landscape produces changes in the structure of that landscape as the landscape adjusts to the new disturbance regime. A computer simulation model was designed to enable analyses of the longterm changes to be expected in landscapes as their disturbance regime changes. The model, DISPATCH, is the first dynamic spatial simulation model built around a geographical information system (GIS). The model also includes a new set of programs, the r.le programs, that is the first set of programs designed for calculating landscape structure measures within a GIS.

The DISPATCH model was used, in a series of experiments, to analyze the effects of human alterations of disturbance regimes (e.g., fire suppression, forest fragmentation) and global change (i.e., warming and cooling) on landscape structure. Landscapes do not adjust quickly to these alterations, requiring 1-2 times the rotation time (the amount of time required for the disturbance regime to disturb a land area equal to the area of a particular landscape), which in landscapes of the western United States and Canada can be several decades to several centuries, based on available data. Landscapes subjected to warming or to longterm fire suppression experience a decline in patch richness, Shannon diversity, the amount of edge and contrast, but an increase in distance between patches, angular second moment (texture measure) and patch size. In contrast, landscapes subjected to cooling, the short-term effects of fire suppression, fragmentation, or traditional prescribed burning tend to respond with increasing richness, Shannon diversity, edge, and contrast, but declining distance, angular second moment, and size. The pattern of response is different at different scales, with important implications for species. 


\section{INTRODUCTION}

This is the final report for the last two years of a three-year project begun at the University of Kansas (DE-FG02-89ER60883), but continued efter year 1 as this contract at the University of Wyoming. That earlier contract produced the following publications, which are the background for this final report:

Baker, W.L. , S.L. Egbert, and G.F. Frazier. 1991. A spatial model for studying the effects of climatic change on the structure of landscapes subject to large disturbances. Ecological Modelling 56: 109-125.

Baker, W.L. 1992. Effects of settlement and fire suppression on landscape structure. Ecology 73(5): 1879-1887.

The first of these publications describes the DISPATCH model and illustrates its use, while the second publication is an application of the DISPATCH model to a specific problem.

\section{OVERVIEW OF RESEARCH COMPLETED}

3.1. The DISPATCH model and r.le programs

The DISPATCH model was described in Baker et al. (1991). The model consists of three major components (Fig. 1). First, there is a driver program written in SIMSCRIPT II.5 (CACl 1987) that runs the model. The driver program contains code for reading in the 


\section{SIMSCRIPT II.5}

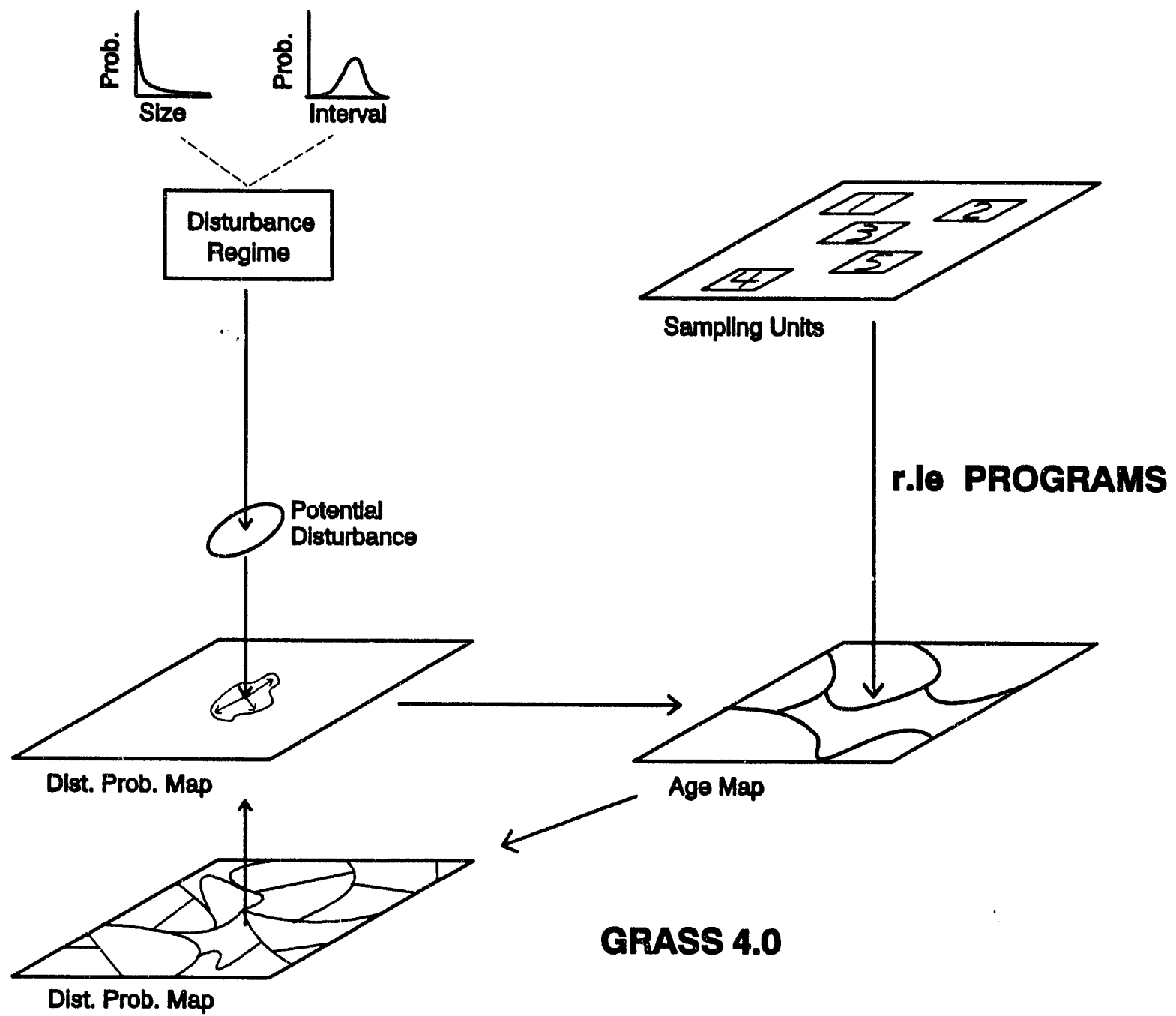


model parameters and keeping track of model processes, including executing the disturbance regime, manipulating the maps of disturbances, and calling the r.le programs. The SIMSCRIPT 11.5 code for the model is in Appendix D. The second component of the model is the GRASS geographical information system (GIS) (USA-CERL 1991), whose GIS function library is extensively used by the driver program, and which is used to store and manipulate the maps of landscape structure.

The third component of the model is the set of r.le programs, which we wrote to enable calculation of landscape structure measures as the model runs, and which requires some explanation. When we began this project there was no existing software for landscape structure analysis which could be called directly from a running simulation model. Indeed, no one had, to our knowledge, ever used a GIS as an integral part of a dynamic simulation model. DISPATCH is the first ecological model to do so, and its development required more effort than we had expected, particularly in creating the r.le programs and linking them with the simulation model.

Creating this set of programs proved difficult, as there were many algorithms that had to be designed and coded that are new GIS algorithms. We had some difficult technical problems to overcome, such as the inability of existing GIS algorithms to quickly and accurately convert a map from raster to vector. This problem alone required us to write difficult new algorithms for raster manipulation of polygon data. For example, we had to develop a new algorithm for determining when a point is within a polygon, a common GIS problem easily implemented in vector format, but one for which there was no algorithm for use with raster data (Laurini and Thompson 1992). Moreover, while we were writing the r.le programs GRASS was released in a major revision that required us to. spend a large part of a year updating the code we had written. 
The result of this substantial effort on the r.le programs is a software package tightly integrated with GRASS that is of general utility for calculating common landscape measures using this GIS either for static analyses or as a component of dynamic simulation models such as DISPATCH. Work on the algorithmic development was a Master's Thesis for Yunming Cai (Cai 1991). The r.le package is described in Baker and Cai (1992) which is reproduced in Appendix A, and the documentation for the package is in Appendix D. The code for the r.le package is available from USA-CERL's ftp server $(129.229 .20 .254)$ in the "incoming" directory (file rle.tar.Z). The programs require GRASS and a Sun Sparcstation with Sun's operating system and Open Windows.

\subsection{The DISPATCH model and simulation of fire suppression}

The DISPATCH model described by Baker et al. (1991) in a conceptual overview was simplified and used to simulate changes in a natural disturbance regime produced by suppression of fires and the restoration of a natural fire regime in an area subjected to fire suppression with support from grant DE-FG02-89ER60883 at the Univ. of Kansas. The initial purpose of these simulations was primarily to use a simple version of the model to see how altering a disturbance regime affected model behavior and model output. The fire record for the Boundary Waters Canoe Area (BWCA) was used as it is one of the finest spatial fire history records available. The BWCA fire record consists of reconstructed maps of fires over three centuries, before and after human alteration of the disturbance regime (Heinselman 1973).

As it turned out, the results from this initial simulation work (Baker 1992) were more interesting than we had expected, and we took the liberty to follow up on these results, when the work was continued at the Univ. of Wyoming, because this modelling 
work was consistent with the general theme of how landscape structure changes as a disturbance regime changes. The result is two additional articles on the fire suppression theme concerning: (1) the effects of fire suppression at multiple scales, and (2) the effects of reinstating a natural fire regime within the BWCA after a period of fire suppression.

The findings from the initial simulation of the effects on the whole BWCA are reported in Baker (1992) and briefly summarized here to provide background for discussing the two articles mentioned above. Fire suppression produced an immediate response in only a few measures of landscape structure (shape, Shannon diversity, and richness), a delay for several decades in the case of two other measures (age, fractal dimension), and a delay for hundreds of years in the case of still other measures (size, angular second moment). In contrast, a brief period of smaller and more frequent burning associated with the settlement period near the BWCA in general produced a stronger and more immediate response.

This led to the idea that the pattern of response of a landscape to alteration of its disturbance regime is governed by the rather odd dynamics of patch populations (Baker 1992). Patch population dynamics is a zero-sum-game in which patch births cause patch deaths, and the birth of larger patches usually means more patch deaths. The result is that both the size distribution of patch births (disturbance size distribution) and the distribution of times between patch births (disturbance interval distribution) control the pattern of response in the landscape.

3.3. The multiscale response to fire suppression

This pattern of response is quite different at different scales, and this has important implications for the species living in landscapes whose disturbance regime is changing. 
The results of simulation experiments to examine the multiscale nature of the response are reported in Baker (1993), which is reproduced in Appendix A. The first significant finding of this research is that as the scale of observation becomes finer, there are more substantial differences (lags) in the timing of a suppression effect when parts of the same landscape are compared. For example, the effect of suppression on mean patch size is lagged $50 \mathrm{yr}$ between the two halves of the BWCA, but as much as $100 \mathrm{yr}$ between the four quarters of the BWCA.

This spatial lag occurs because the location of a suppression effect is really restricted to the region that would have burned had suppression not occurred, and this area radiates out from the location of an ignition. Thus the parts of the iandscape that respond most rapidly to suppression are the places where post-suppression fires initiate. The remainder of the landscape remains unchanged. There is reason to believe that this result is very general; that is, as a disturbance regime shifts, the part of the landscape that responds most rapidly to the shift is the part that receives the new disturbances. The remainder of the landscape may remain adjusted to the old disturbance regime.

An important implication of this finding is that species with different home ranges or ecological neighborhoods will experience different patterns of change in the same landscape. Species that have small neighborhoods may experience rapid changes if their territories are within those parts of the landscape that respond immediately; however, these species should be able to locate habitat refugia in the parts of the landscape that have not changed. In contrast, species that have larger home ranges will tend to average out the spatially heterogeneous consequences of alteration in the disturbance regime, but they will not be able to find refugia as easily. 


\subsection{The restoration experiment}

This purpose of this experiment was to see whether a landscape subjected to fire suppression would return to its pre-suppression landscape structure if the pre-suppression disturbance regime were reinstated. The results of this experiment are reported in Baker (in review), which is reproduced in Appendix B. The results suggest that the altered landscape structure produced by 82 years of fire suppression can be generally restored within 50-75 years after reinstatement of the pre-suppression disturbance regime. However, some measures responded more rapidly than did others.

\subsection{The synthesis paper using the DISPATCH model}

The simulation research with fire suppression suggested that landscapes might respond in an similar manner to similar changes in the disturbance regime, regardless of the source of the change (e.g., global warming, forest fragmentation), and a final set of experiments was undertaken to try to derive a general theory about the response of landscapes to alterations in their disturbance regimes. The results of this set of experiments are summarized in a paper submitted to Landscape Ecology, and reproduced in Appendix B. In this synthesis work we compared the results of simulating climatic changes (global warming and cooling) with the effect of human land uses (fragmentation and restoration) on the structure of a generalized temperate-zone forested disturbance landscape over 400 years.

We found that landscapes require $1-2$ rotations (defined as the time required to disturb an area equal to the area of the particular landscape) to adjust to a new disturbance regime no matter how the disturbance regime is changed. However, the structure of the new landscape is strongly controlled by both the size and interval 
distributions of the new disturbance regime. We found that the density of patches in the initial landscape had relatively little effect on the rate of adjustment. The result is that new disturbance regimes that decrease the rotation time, either because of larger disturbances or more frequent disturbances (or both), will result in more rapid adjustment in landscape structure than will regimes that increase the rotation time. It matters little whether the alteration in the disturbance regime comes from changes in climate or human land uses, so that there is a set of disturbance regime alterations that can be expected to produced similar patterns of change in landscape structure (Fig. 2). The actual amount of change in a particular measure and the comparative order in which different measures respond are determined by the relative amounts of change in the disturbance size and interval distributions. Two landscapes with the same rotation time will adjust in about the same time period, but may end up with quite different structures depending on the characteristics of these two distributions.

\section{RESEARCH STILL IN PROGRESS}

4.1. A physically-based model of flood disturbance on the Animas River We collected extensive field data from the Animas River in southwestern Colorado's San Juan Mountains with the goal of developing a physically-based model of the effects of climatic fluctuations on the landscape structure produced by floods along this river. This model was to have been a refinement of the DISPATCH model for a specific location and disturbance regime. We used tree-ring analysis to reconstruct the year of origin of 86 flood-produced patches found along a $6 \mathrm{~km}$ reach of this river, and we mapped and 
KINDS OF CHANGES:

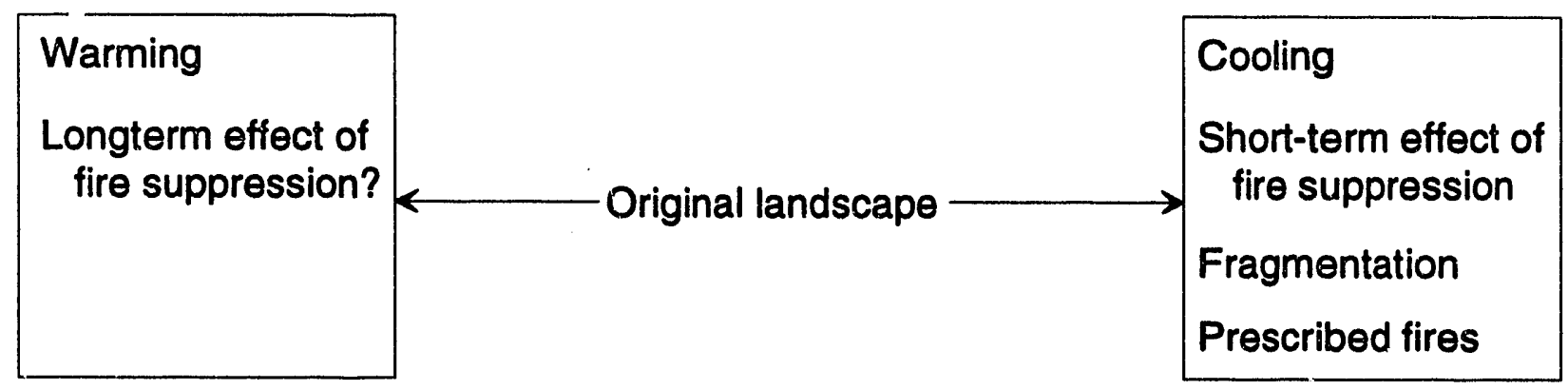

EFFECT ON DISTURBANCE REGIME:
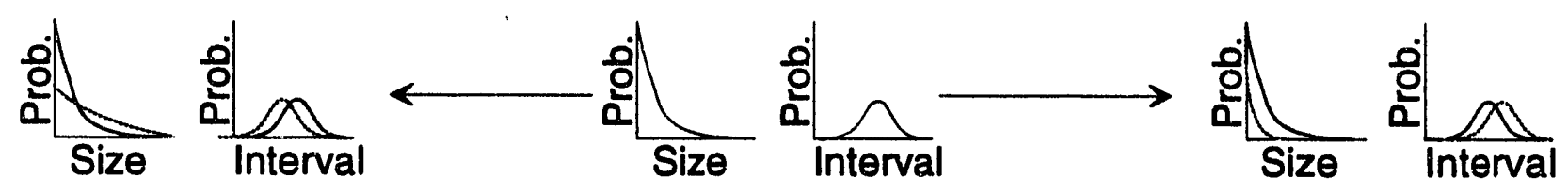

EFFECT ON LANDSCAPE STRUCTURE:

Lower Richness

Lower Shannon Diversity

Less Edge

Less Contrast

Larger Distance

Larger Ang. Sec. Mom.

Larger Size
Higher Richness

Higher Shannon Diversity

More Edge

More Contrast

Smaller Distance

Smaller Ang. Sec. Mom.

Smaller Size 
digitized the boundary of each patch based on scanned aerial photography. We also collected data on the vegetation composition of each patch and its tree population structure. We surveyed 36 valley cross-sections along the reach and then attempted to use the GRASS GIS to construct a digital elevation model (DEM) of the reach. We used the cross-sectional data with the U.S. Army Corps of Engineers' HEC-2 model to reconstruct water surface elevations at each of the 36 valley cross-sections for 20 levels of streamflow spanning the range of historical streamflow, based on U.S.G.S. data from the Durango gauging station downstream of the study reach. We planned to use the GIS to produce interpolated maps of water depth for the reach for each of the 20 levels of discharge, and then to use these maps to determine the stream power that produced each of the $\mathbf{8 6}$ mapped disturbances. The final link was to reconstruct the regional synoptic weather pattern present on each day that produced one of the mapped disturbances.

From these data sets, we planned to calibrate a physical model that could be used to simulate changing synoptic weather patterns, the occurrence of floods, and the physical process by which an existing forest stand along the reach is destroyed as stream power exceeds the resisting ability of the trees. This model would then have been run with different climatic scenarios and the changing pattern of landscape structure would have been monitored with the r.le programs. This would enable an assessment of the possible changes in landscape structure along the river reach that might accompany climatic change.

However, we expended considerable time trying to develop a reasonable DEM for the reach from the 36 surveyed valley cross-sections, and this critical step was unsuccessful. As it turns out there are no existing algorithms that we could find that can adequately create a DEM from the kind of cross-sectional survey data that are traditionally 
used to quantify stream morphology. Our mistake was to follow these traditional techniques. The problem is that cross-sections are traditionally placed in the center of rather uniform subreaches that have a consistent cross-sectional morphology. This does not produce a data density along the longitudinal profile that is comparable to the data density along the cross-section. Common interpolation algorithms, including quadrant specific algorithms, cannöt adequately interpolate the longitudinal profile from these kinds of data. A dense, somewhat evenly spaced grid of points, or mapped contour lines is what is needed. Another problem is that considerable precision is needed in the interpolation as water depths at high discharge along this reach may only be 1-2 $\mathrm{m}$.

We were very frustrated by this problem, but now know that a more extensive survey will be needed using a global positioning system. We are not giving up on completing this modelling effort as we believe it has potential to be very interesting, but funding to complete this research will have to be obtained elsewhere, and unfortunately the physically-based model and simulations cannot be completed until we can construct a precise DEM for the river reach.

\subsection{Vegetation patterns and succession along the Animas River}

As mentioned above, we collected data on the vegetation composition and tree population structure within each patch along the Animas River study reach. A Master's thesis is in progress, by Gillian Walford, to analyze these data. This thesis will consist of two publishable articles. The first is an analysis of vegetation variation along the reach in relation to the geomorphology of the river produced by disturbance. The second is an analysis of successional patterns along the reach following disturbance. A significant finding of this research is that a few of the disturbances have had much more effect than 
the others. One of the disturbances, a major flood in 1927 A.D., may have completely changed the pattern of succession along the river reach. The implication of this is that not all disturbances are of equal importance. Modelling the effect of climatic change on disturbance regimes may be most meaningful from the species standpoint if focused on the kinds of disturbances represented by the 1927 flood, which resulted from a tropical cyclone that crossed Mexico and Arizona and then stalled against the San Juan Mountains.

This thesis research will provide a link between the modelling of landscape structure and the plant species that live in, and presumably are adapted to the changing structure of this disturbance landscape.

\section{BIBLIOGRAPHY}

Baker, W.L. 1992. Effects of settlement and fire suppression on landscape structure. Ecology 73: 1879-1887.

Baker, W.L. 1993. Spatially heterogeneous multi-scale response of landscapes to fire suppression. Oikos 66: 66-71.

Baker, W.L., in review. Spatial modeling of the restoration of landscape structure altered by fire suppression. Environmental Management, in review.

Baker, W.L. and Y. Cai. 1992. The r.le programs for multiscale analysis of landscape structure using the GRASS geographical information system. Landscape Ecology 7: 291-302.

Baker, W.L., S.L. Egbert, and G.F. Frazier. 1991. A spatial model for studying the effects of climatic change on the structure of landscapes subject to large disturbances. 
Ecological Modelling 56: 109-125.

CACl, Inc. 1987. SIMSCRIPT II.5 programming language. CACI Products Co., La Jolla, CA.

Cai, Yunming. 1991. GLE-Landscape ecological analysis software using the GIS GRASS. Master's thesis, University of Wyoming, Laramie, Wyoming. $137 \mathrm{pp}$.

Heinselman, M.L. 1973. Fire in the virgin forests of the Boundary Waters Canoe Area, Minnesota. Quat. Res. 3: 329-382.

Laurini, R. and D. Thompson. 1992. Fundamentals of spatial information systems. Academic Press, London.

USA-CERL. 1991. GRASS 4.0 user's reference manual. United States Army Construction Engineering Research Laboratory, Champaign, Illinois. 
6. APPENDICES 
APPENDIX C. SIMSCRIPT CODE FOR THE DISPATCH MODEL 


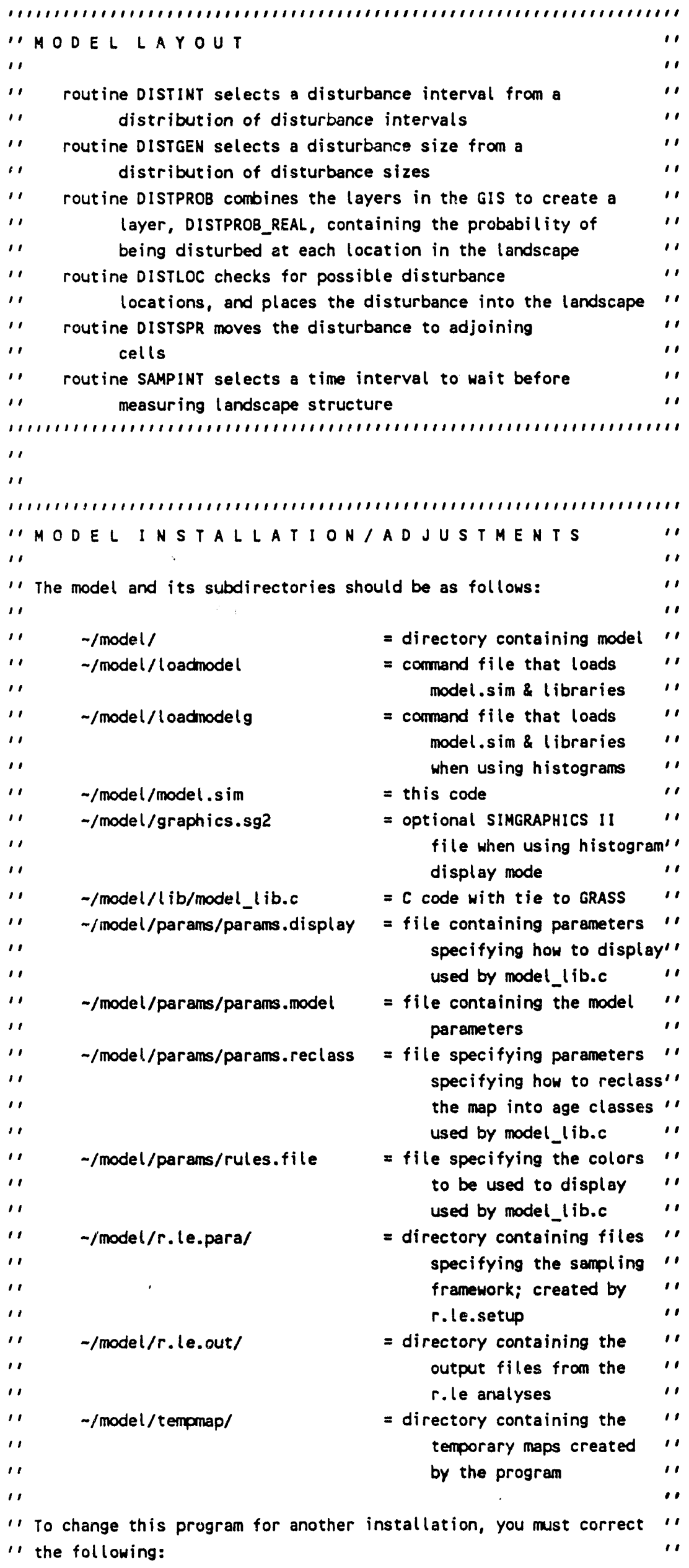




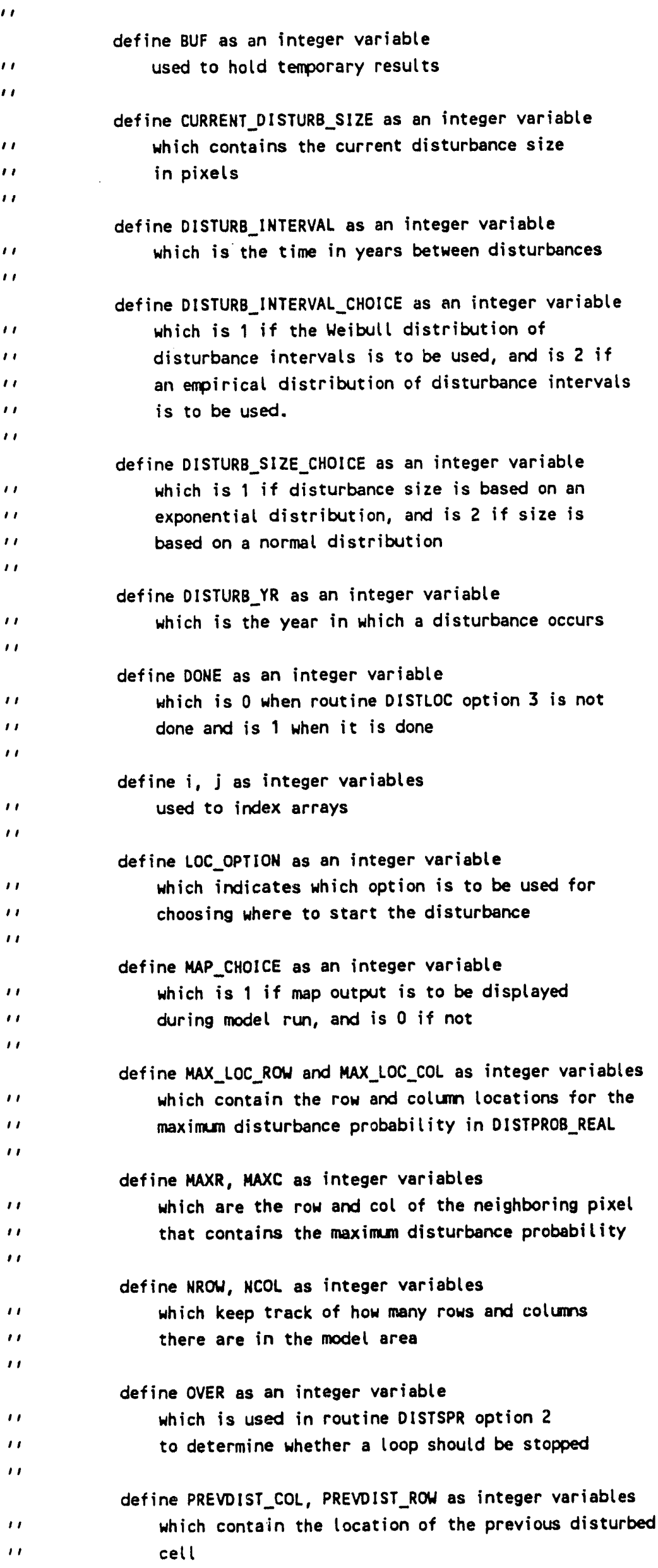


define $R, C$ as integer variables which contain the row and col location as the disturbance spreads from pixel to pixel

define SAMP_INTERVAL as an integer variable which is the current time to wait before measuring landscape structure

define LAST_SAMP_YR as an integer variable which is the year the landscape structure is measured

define LAST_SAVE_YR as an integer variable which is the year the map was last saved

define SAVE_GAP as an integer variable which is the number of years to skip before saving a new copy of the AGE layer in the GRASS GIS

define SPREAD_OPTION as an integer variable which contains 1 for option 1 or 2 for option 2

define START.YR, STOP.YR as integer variables which are the starting and stopping years for the simulation run

define STM_SIZE, STM_DISTURB, STM_LOC, STM_SAMP, STM_SPREAD as integer variables which specify the random number stream to use in picking random numbers for disturbance size, disturbance interval, disturbance location, sampling interval, and disturbance spread

define TEMP_YEAR as an integer variable which holds the YEAR for display

define TIES as an integer variable which is the size of arrays TIESROW and PROBTIESROW

define YEAR as an integer variable which keeps track of the current year in the model run

define ZEROTIES as an integer variable which keeps track of the number of zeros among the 8 neighbors during the spread process

\section{REAL VARIABLES}

define DISTMax as a real variable which indicates the maximum potential value in the GRASS DISTPROB layer

define DISTPROB_SUM as a real variable which contains the cumulative sum of the disturbance probabilities in the DISTPROB_REAL layer 
routine DISTSPR option 2

define DISTURB_SIZE as a real variable which contains the size of the disturbance

define MAX as a real variable which contains the value of the maximum disturbance probability in the 8 neighboring pixels

define MAX OISTPROB as a real variable which contains the maximum disturbance probability in DISTPROB_REAL. Used to find the location for initiating a disturbance.

define MINIMUMDIST as a real variable which is the minimum probability that must occur in order for a disturbance to be initiated. This is used with option 2 in routine DISTLOC

define RAND_LOC_VAL as a real variable which contains the random value selected from the cumulative sum of disturbance probabilities in routine DISTLOC option 3

define RAND_SPR_VAL as a real variable which contains the random value selected from the cumulative sum of disturbance probabilities in the 8 neighbors in routine DISTSPR option 2

define SAMP_MEAN as a real variable which contains the mean interval between measurements of landscape structure

define SAMP_STDEV as a real variable which contains the standard deviation of the mean interval between measurements of landscape structure

define SIZE_EXPMEAN as a real variable which contains the mean of the exponential distribution of potential disturbance sizes

define SICE_NORMEAN as a real variable which contains the mean of the normal distribution of potential disturbance sizes

define SIZE_NORSTDEV as a real variable which contains the standard deviation of the normal distribution of potential disturbance sizes

define TEMP_INTERVAL as a real variable which temporarily holds MEAN.DISTURB_INTERVAL for display

define TEMP_SIZE as a real variable which temporarily holds MEAN.DISTURB_SIZE for display 
which contains the Weibull scale parameter value. This value is the value below which approximately $63 \%$ of the values in the distribution will lie

define HEIBULL_SHAPE as a real variable which contains the Weibull shape parameter value. This value ranges from 1.0 for an approximately negative exponential distribution to 3.6 for an approximately normal distribution to large values (e.g. 20.0) for a highly negatively skewed distribution

\section{TEXT VARIABLES}

define DISTURB_OUT as a text variable which contains the name of the output file for the history of disturbances that occur during the model run

define DISTURB_TEMP as a text variable which contains the word OISTURB, which is the prefix for al! UISTURB_OUT files

define FORMULA as a text variable which contains the formula for calculating disturbance probabilities

define GLEDIST as a text variable which contains the r.le.dist landscape stucture analys is command and its parameters

define GLEPATCH as a text variable which contains the r.le.patch landscape stucture analys is command and its parameters

define GLETEX as a text variable which contains the r.le.dist landscape stucture analys is command and its parameters

define INIT_MAP as a text variable which contains the name of the starting map layer

define PARAMS_FILE_NAME as a text variable which contains the name of the file containing the model input parameters

define RUN as a text variable which contains the 10 for the run

define RUN_YR as a text variable which contains the concatenation of RUN and YEAR

define YR_TXYT as a text variable which is used temporarily during concatenation of RUN and YEAR 
which is a map of the disturbance location

define DISTPROB_CUM as a real, 2-dimensional array which is an array containing the cumulative sums of cisturbance probabilities in array DISTPROB_REAL

define DISTPROB_INT ac an integer, 2-dimensional array which is an array containing disturbance probabilities

define OISTPROB_REAL as a real, 2-dimensional array which is an array containing relativized disturbance probabilities

define PROBTIESCOL as an integer, 1-dimensional array which is the same as TIESCOL but used with option 2 in routine DISTLOC.

define PROBTIESROW as an integer, 1-dimensional array which is the same as TIESROW but used with option 2 in routine $D$ !STLOC.

define TIESCOL as an integer, 1-dimensional array which holds colum indices which are currently tied as having the miximum probability of disturbance. Used with option 1 in routine DISTLOC.

define TIESROW as an intiger, 1-dimensional array

which holds row indices which are currently tied as having the maxinum probalility of disturbance. Used with option 1 in routine DISTLOC.

define ZEROTROW as an integer, 1-dimensional array which holds row indices which are currently tied as having a zero value in the disturbance probability map.

define ZEROTCOL as an integer, 1-dimensional array which holds colum indices which are currently tied as having a zero value in the disturbance probability map.

\section{RANDOM STEP VARIABLES}

the system has an EMPIR_INTERVAL random step variable define EMPIR_INTERVAL as an integer variable which contains the empirical distribution of the number of years between disturbances

FUNCTIONS

define CAN_CHECK as integer function which keeps the disturbance from spreading off the map or onto pixels that have al ready been disturbed 
tally FREQ.DISTURB_INTERVAL ( 0 to 15 by 1 )

as the dynamic histogram of OISTURB_INTERVAL

tally FREQ.DISTURB_SIZE ( 0 to 3000 by 200)

as the dynamic histogram of DISTURB_SIZE

tally MEAN.DISTURB_INTERVAL as the mean of D!STURB_INTERVAL

tallY MEAN.DISTURB_SIZE as the mean of DISTURB_SIZE

1

11

1 DISPLAY VARIABLES

1

display variables include TEMP_YEAR, TEMP_SIZE, TEMP_INTERVAL

11

11

end

1,

main

11

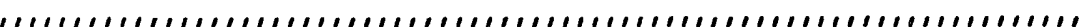

“READIN NAME OF PARAMETERS FILE

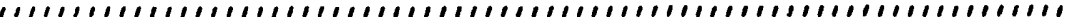

print 1 line thus

TYPE NAME OF PARAMETERS FILE:

read PARAMS_FILE_NAME

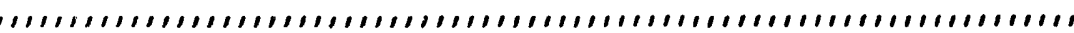

$\because$ READIN PARAMETERSANDRESERVESPACE.

$11,1,1,1,1,1,1,1,1,1,1,1,1,1,1,1,1,1,11,1,1,11,1,1,1,1,1,1$

"This section of the program reads in the model parameters

1' from the parameters file. These parameters are:

Line 1: $\quad x x x x \quad x x x x$

Starting year and stopping year

for model run

Line 2: $\quad x x x x \quad x x x x$

Row and colum dimensions (in

pixels) of the model area

Line 3: $X X X X X X X X$

Random number streams for the:

(1) size distribution

(2) disturbence interval distribution

(3) location algorithm DISTLOC

(4) sampling interval distribution

(5) disturbance spread

Line 4: $X . . \times(20$ chars) Run identification

Line 5: $X . . \times(20$ chars) Name of starting map layer

Line 6: $x$

Display map output during the model run?

1 - do display

2 - don't display

Line 7: $\quad x x x \quad x x x \quad x x x$

(1) Mean for the exponential size 


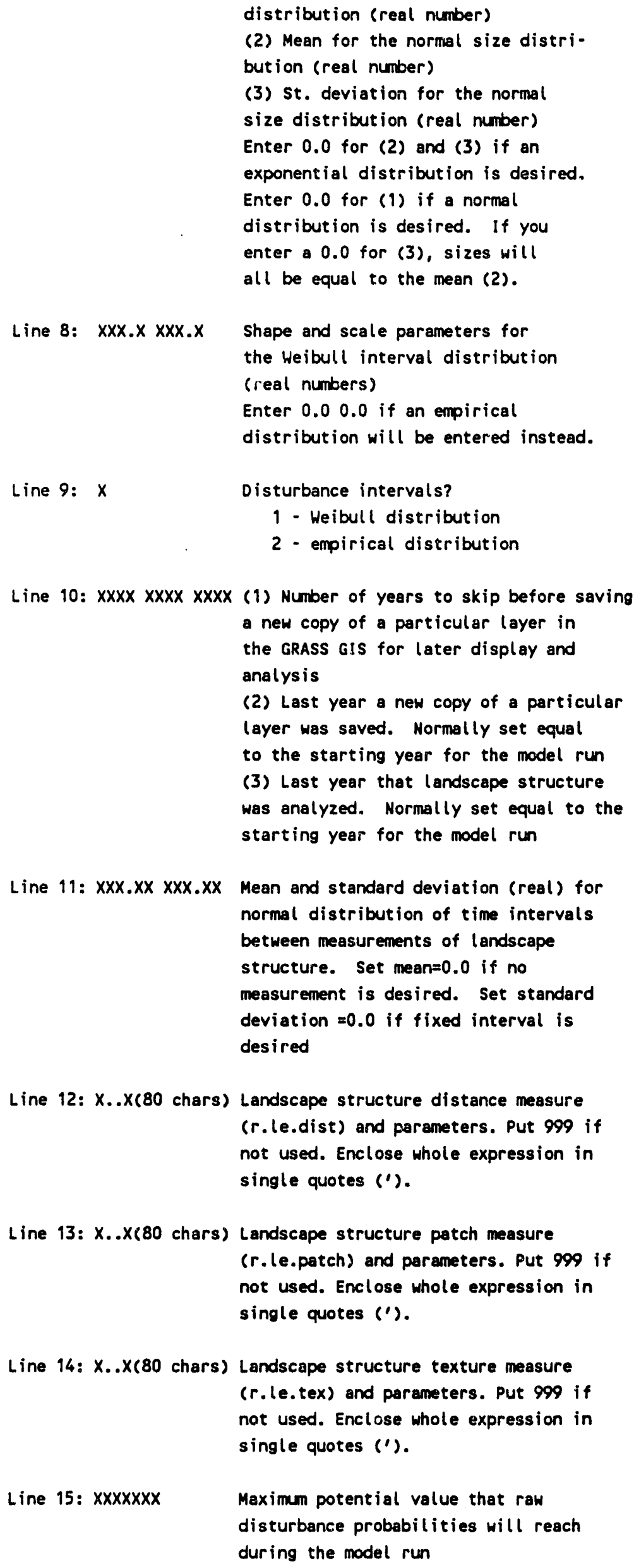

Line 10: $X X X X X X X X X X X X$ (1) Number of years to skip before saving a new copy of a particular layer in the GRASS GIS for later display and analysis

(2) Last year a new copy of a particular layer was saved. Normally set equal to the starting year for the model run (3) Last year that landscape structure was analyzed. Normally set equal to the starting year for the model run

Line 11: $x x x . X X X X X . X X$ Mean and standard deviation (real) for normal distribution of time intervals between measurements of landscape structure. Set mean $=0.0$ if no measurement is desired. Set standard deviation $=0.0$ if fixed interval is desired

Line 12: X..X(80 chars) Landscape structure distance measure (r.le.dist) and parameters. Put 999 if not used. Enclose whole expression in single quotes (').

Line 13: $x . . \times(80$ chars) Landscape structure patch measure (r.le.patch) and parameters. Put 999 if not used. Enclose whole expression in single quotes (').

Line 14: $X . . \times(80$ chars $)$ Landscape structure texture measure (r.le.tex) and parameters. Put 999 if not used. Enclose whole expression in single quotes (').

Line 15: $x x x x x x x \quad$ Maximum potential value that raw disturbance probabilities will reach during the model run 
Line 18: $x$

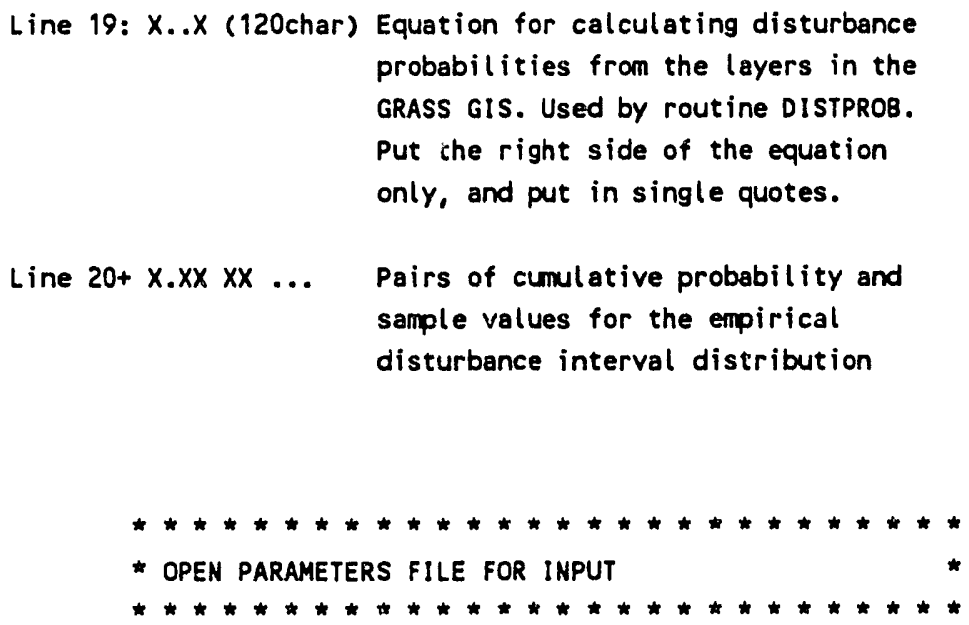

open unit 1 for input, recordsize is 800 ,

file name is PARAMS_FILE_mAME

use unit 1 for input

if ropenerr. $v$ < 0

print 1 line thus

Unable to ope:l parameters file for input close unit 1

always in a probabilistic manner
Minimum disturbance probability to be used with option 2 for locating disturbances

Option for locating disturbances:

1 - the disturbance is located randomly among locations that have tied disturbance probabilities

2 - the disturbance is located randomly among locations that have a minimum probability of being disturbed

3 - the disturbance is located based on disturbance probabilities

Option for spreading disturbances:

1 - the disturbance is spread to the pixel, among the 8 neighbors, that contains the highest disturbance probability.

2 - the disturbance is spread probabilistically, according to the disturbance probabilities of the adjoining 8 neighbors. GRASS GIS. Used by routine DISTPROB. Put the right side of the equation only, and put in single quotes.

Pairs of cumulative probability and sample values for the empirical disturbance interval distribution 
read START.YR, STOP.YR

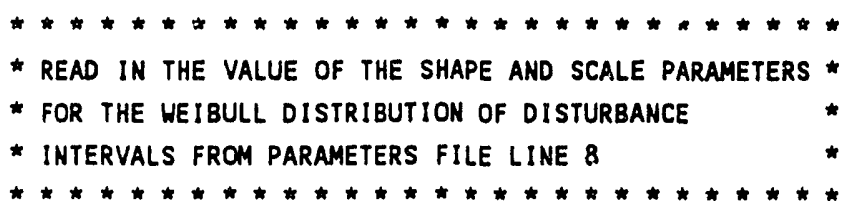




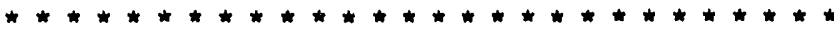

* read in the choice betheen using a heibull distri-

* BUTION (DISTURB_INTERVAL_CHOICE = 1) OR AN

* empirical distribution coisturb_interval_choice = *

* 2) of disturbance intervals from parameters file

* LINE 9

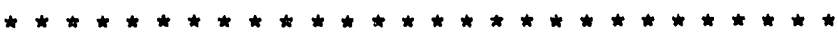

read DISTURB_INTERVAL_CHOICE

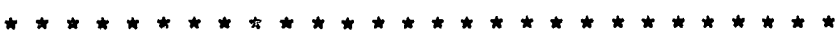

* read In how many years to skip before saving * *

* a layer in grass from parameters file line 10 * *

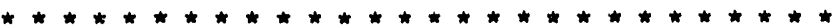

read SAVE_GAP, LAST_SAVE_YR, LAST_SAMP_YR

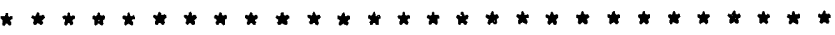

* read in the mean and standard deviation for a

* normal distribution of time intervals between *

* measurements of landscape structure from parameters *

* File line 11

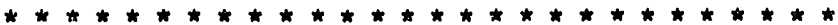

read SAMP_MEAN, SAMP_STDEV

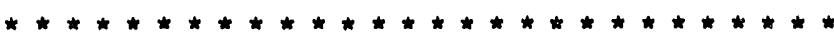

* read IN the landscape structure measure gle.dist *

- and ItS parameters from parameters file line 12

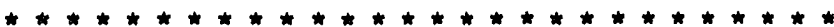

read GLEDIST as T *

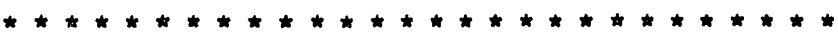

* read in time landscape structure measure gle.patch *

* and ItS parameters from parameters file line 13 *

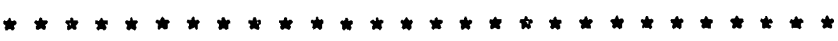

read GLEPATCH as $T$ *

* read in the landscape structure measure gle.tex

* aND ITS PaRAMETERS fROM PARAMETERS fILE LINE 14 *

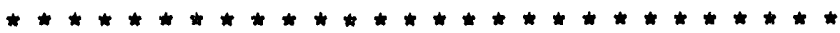

read GLETEX as $T$ *

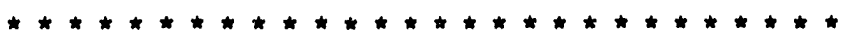

* read in the maximum potential value for distprob

* from parameters file line 15 
reserve DISTPROB_CUM(*,*) as NROW by NCOL reserve DISTPROB_INT(*,*) as NROW by NCOL reserve DISTPROB_REAL(*,*) as NROW by NCOL reserve DISTMAP $\left({ }^{\star},{ }^{*}\right)$ as NROW by NCOL reserve PROBTIESROW(*) as (NROH * NCOL) reserve PROBTIESCOL (*) as (NROW * NCOL) reserve TIESROW(*) as 8 reserve TIESCOL (*) as 8 reserve ZEROTROW(*) as 8 reserve ZEROTCOL(*) as 8 
always

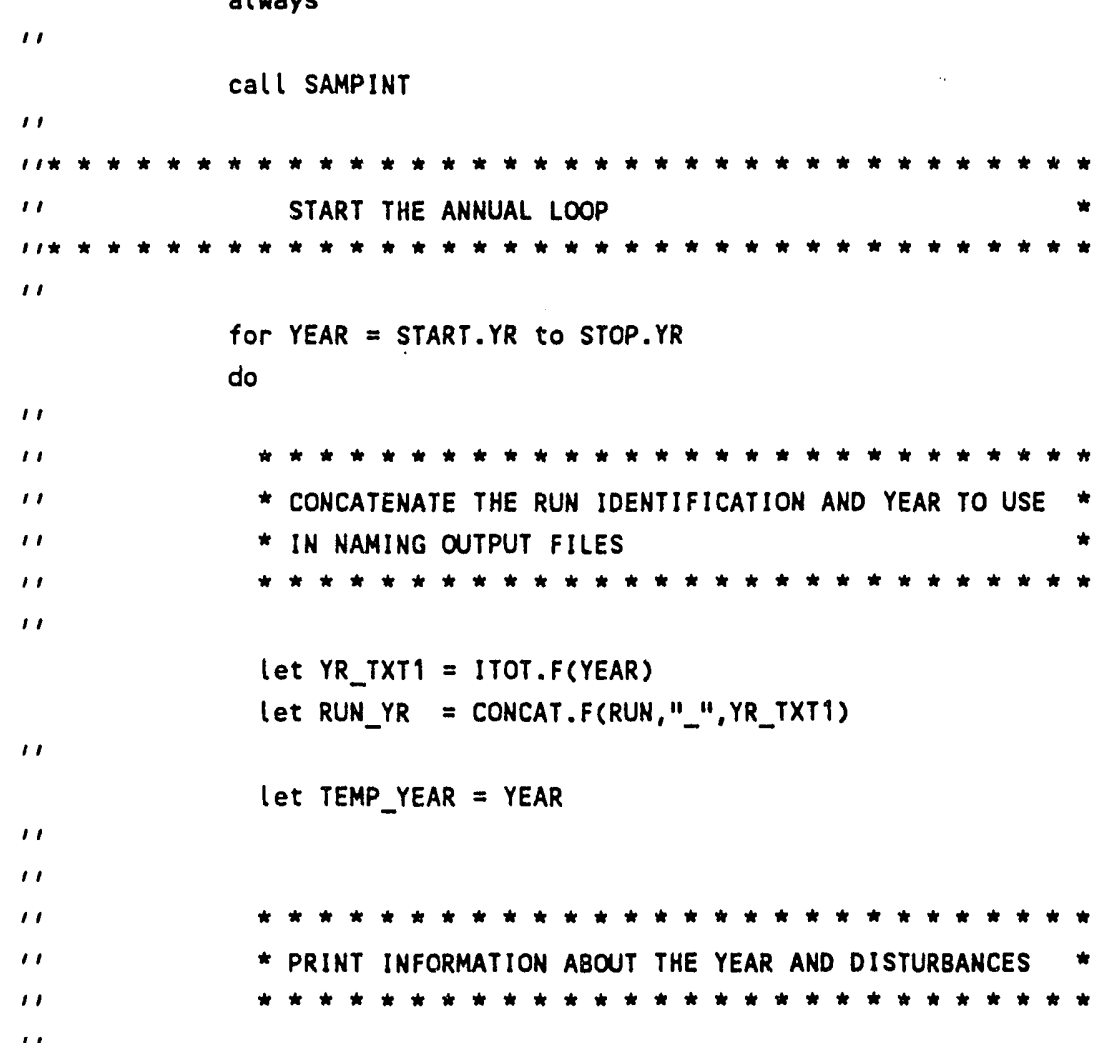

print 2 lines with YEAR, DISTURB_YR, DISTURB_INTERVAL thus

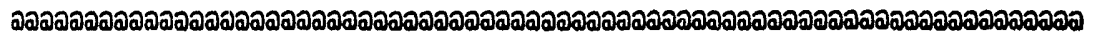

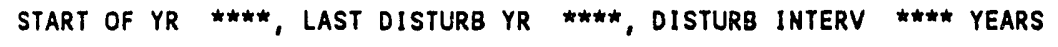
, II 
let TEMP_SIZE = MEAN.DISTURB_SIZE

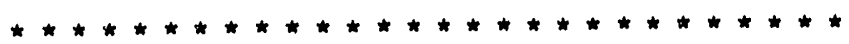

$\star 2$. Print the disturbance size

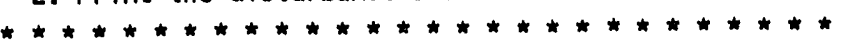

print 1 line with OISTURB_SIZE thus

$$
11
$$

11

11
11
11
11
11

*3. Write the disturbance year, disturbance size, * and disturbance interval to a file

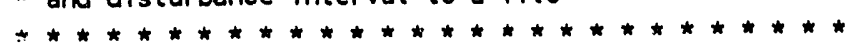

$$
\begin{aligned}
& \text { Write YEAR, DISTURB_SIZE, DISTURB_INTERVAL as } \\
& \text { I } 4, S 2, D(8,1), S 2,14,1 \text { using unit } 2
\end{aligned}
$$
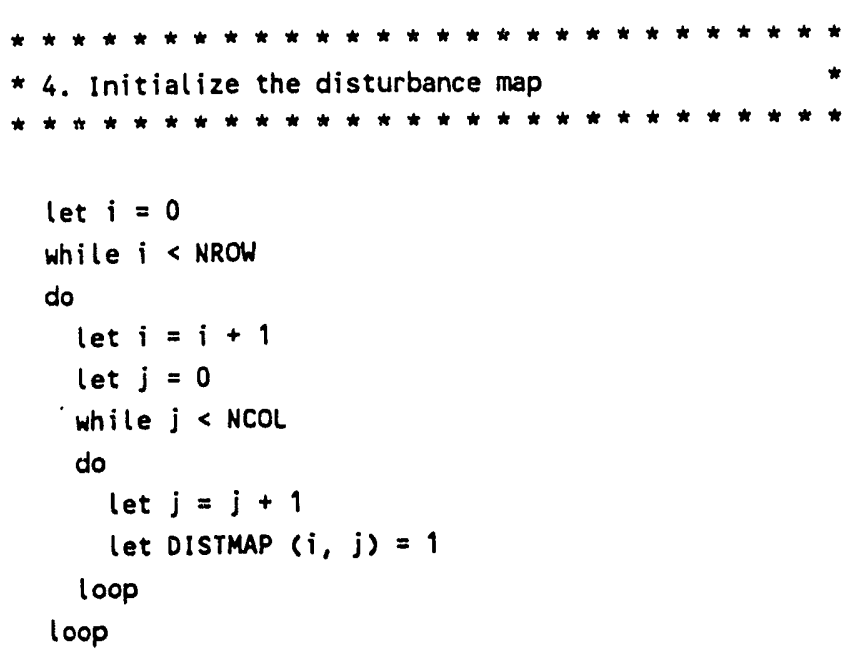

* 5. Calculate the disturbability of the landscape

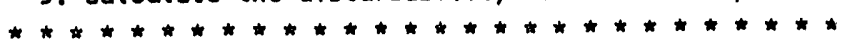

call DISTPROB

* 6. Locate the disturbance in the landscape.

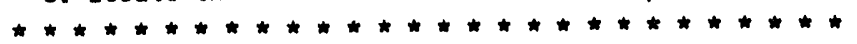

call DISTLOC

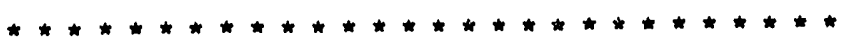

*7. Check to see if DiSTPROB contains only 0's. If *

* so, do nothing. If DISTPROB contains disturbable *

* pixels, then spread the disturbance 
if $R=0$

print 1 line thus

DISTPROB ALL O'S-NO DISTURBANCE

else

call DISTSPR

* 8. Update the age layer in the GRASS GIS to reflect* * the effects of the disturbance. Use the following * * external routines to do this:

* a. call putnum writes distmap.ascii, an ASCII

* version of the Distmap array.

* b. call write_asci $i$ reads in distmap.ascii, and *

* writes out distmap_whdr.ascii, which *

* contains the header information needed

* to read DISTMAP data into GRASS.

* c. call update_map reads distmap_whdr.asci i into* * GRASS using the GRASS Mimportcell * * cormand, and then uses the Gmapcalc * * cormand to change all the disturbed * * locations in the AGE map to AGE=0 *

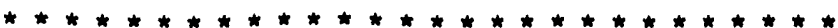

for $i=1$ to NROW

do

for $j=1$ to NCOL

do

call putnum (DISTMAP $(i, j)$, NROW, $j, i)$ loop

loop

call write_ascii

call update_map

always

11

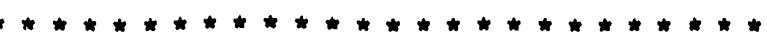

* 9. Determine the next disturbance interval, then *

* use a temporary variable to produce the display of *

* cumulative mean disturbance interval if the choice *

* was made to display graphic output during the model *

* run

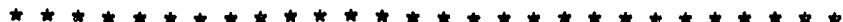

Call DISTINT

let TEMP_INTERVAL = MEAN.DISTURB_INTEPYYAL 


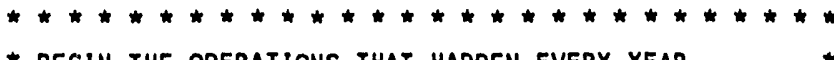

* 8egin the operations that happen every year

* regardless of disturbance. these include:

* 1. display the last disturbance map and the *

* current map if graphic output was *

* requested ouring the mooel run *

* 2. save the map for later analysis, if the *

* interval to skip before saving has passed *

* 3. add a year to the age of each pixel in the *

* age layer of the grass gis *

* 4. print information about sampling *

* 5. analyze landscape structure if it's time *

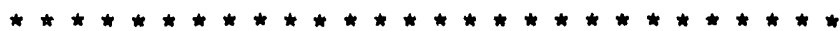

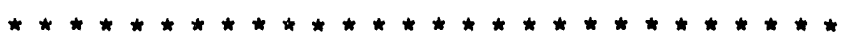

* 1. Display the last disturbance map and the current * * age map if graphic output was requested during the *

* mrdel run. Use external routines to do this

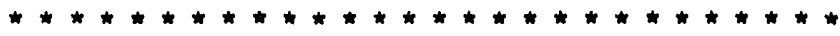

if MAP_CHOICE $=1$

call reclass_map

call show_map given RUN_YR

always

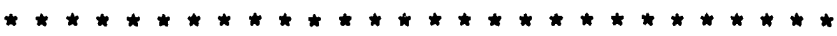

2. Save a map for later analysis, if the time $\star$

* interval to skip before saving has passed. Use an *

* external routine to save the map.

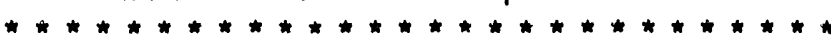

if ((YEAR-LAST_SAVE_YR: = SAVE_GAP)

call save_grass_layer given RUN_YR

let LAST_SAVE_YR = YEAR

al ways

11

11

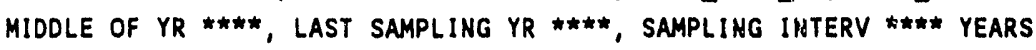

*3. Add a year to the age of each pixel in the age * layer of the GRASS GIS. Use an external routine * * called annual_updt, which uses the GRASS cmapcalc * * command to add 1 to every pixel in the AGE map

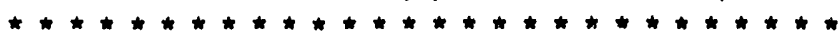
call annual_updt

$*$ 4. Print information about sampling

print 1 line with YEAR, LAST_SAMP_YR, SAMP_INTERVAL thus $\star$ II , 


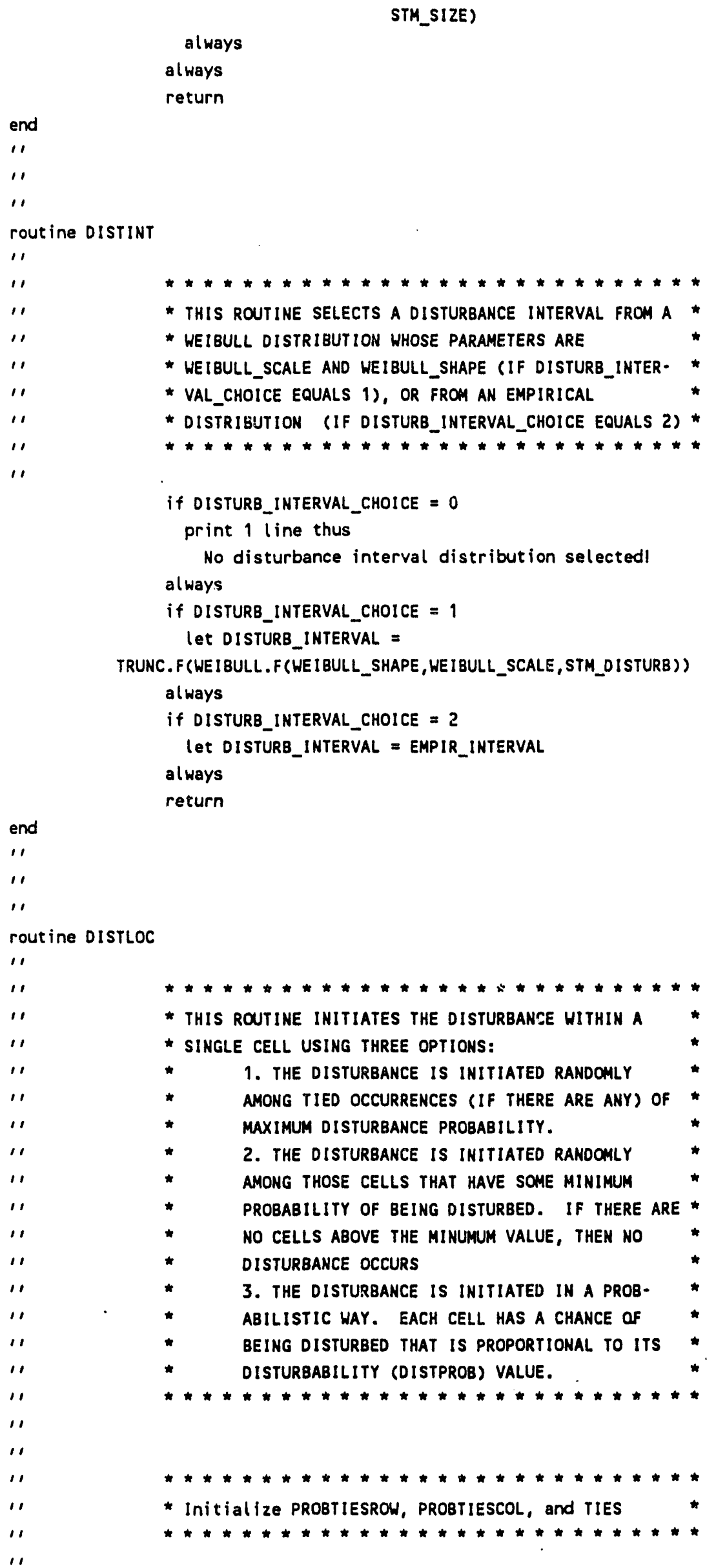

for $i=1$ to (NROW * NCOL)

do 


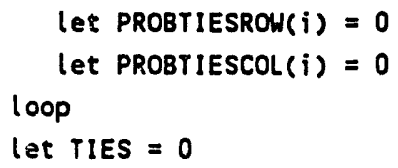

b. Search the disturbance probability array for the maximum value

while $i<$ NROW

do

$i=i+1$

$j=0$

while $j<$ NCOL

do

$$
j=j+1
$$

c. Then set that location in the disturbance map layer, DISTMAP, equal to 0 , unless there are ties. If so, then use the random number generator to choose anong the ties

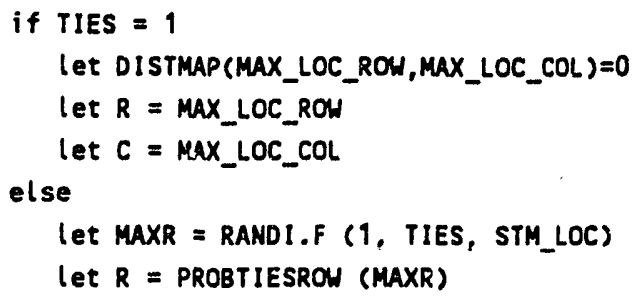


let $C=$ PROBTIESCOL (MAXR)

let $\operatorname{DISTMAP}(R, C)=0$

always

always

* Option number 2

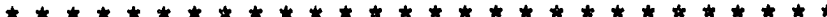

if LOC_OPTION $=2$

a. Initialize the variables

TIES $=0$

$i=0$

b. Search the disturbance probability array for all cells that have a value above the minimum value for disturbance

while $i<$ NROW

do

$\mathbf{i}=\mathbf{i}+\mathbf{1}$

$j=0$

while $j<N C O L$

do

$j=j+1$

if DISTPROB_REAL $(i, j)>$ MINIMUMDIST

let TIES $=$ TIES + 1

let PROBTIESROW(TIES) $=\mathbf{i}$

let PROBTIESCOL(TIES) $=j$

always

loop

loop

c. If there are no ties, then no location is disturbable. Otherwise choose randomly among the tied locations.

if TIES $=0$

let $R=0$

let $C=0$

else

let MAXR = RANDI.F (1, TIES, STM_LOC)

let $R=$ PROBTIESROW (MAXR)

let $C=$ PROBTIESCOL (MAXR)

let $\operatorname{DISTMAP}(R, C)=0$

always

always

- Option number 3

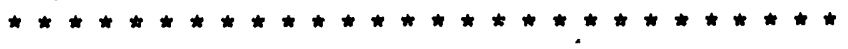

if LOC_OPTIOH = 3 


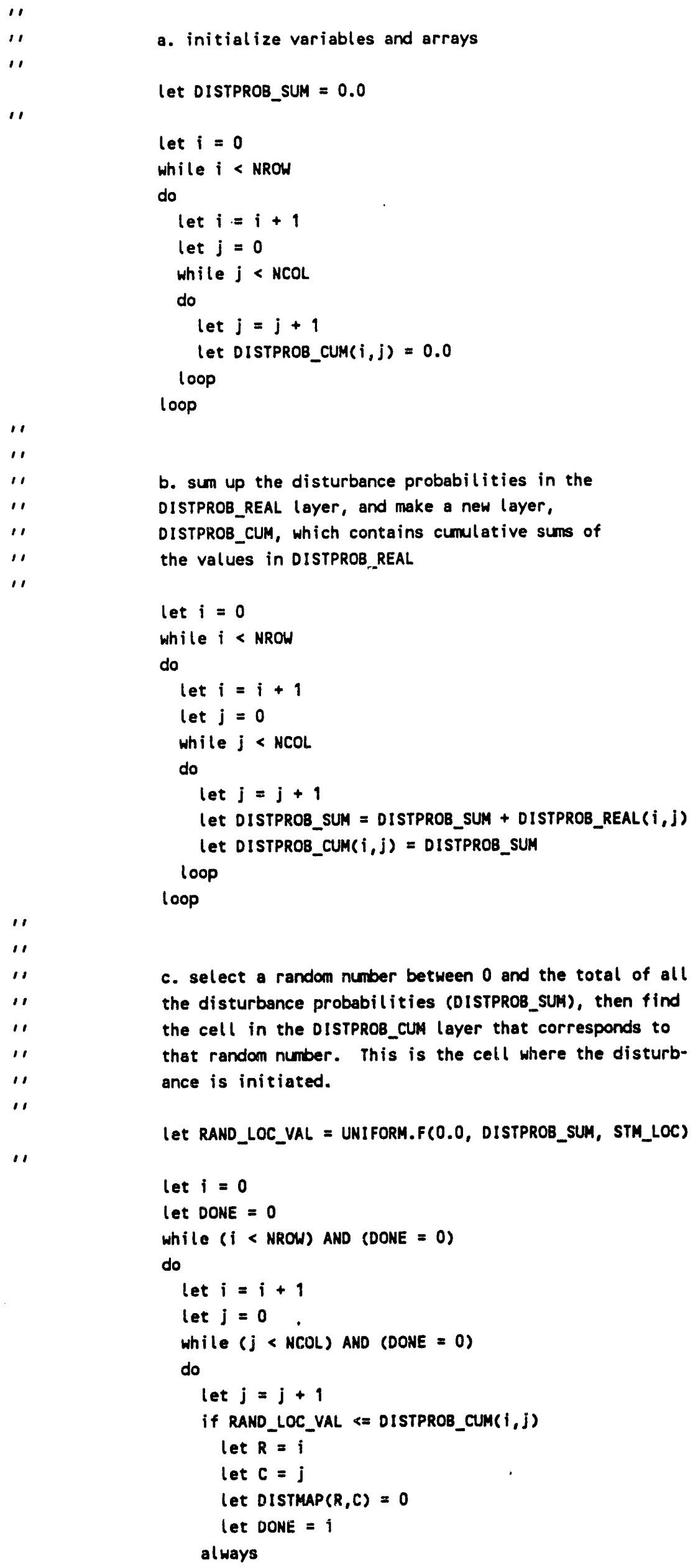

c. select a random number between 0 and the total of all the disturbance probabilities (DISTPROB_SUA), then find the cell in the OISTPROB_CUM layer that corresponds to that random number. This is the cell where the disturbance is initiated.

let RAND_LOC_VAL $=$ UNIFORM.F $(0.0$, DISTPROB_SUM, STM_LOC $)$ 
11

always

return

end

il

11

11

routine DISTPROB

,

11

1. Call the external routine make_distprob to read the equation for calculating disturbance probabilities, to use the GRASS Gmapcalc cormand to calculate disturbance probabilities, to create a new GRASS layer called DISTPROB, and to write it out as an ASCII file called distprob.ascii

call make_distprob given FORMULA

2. Read in DISTPROB_INT from unit 10. Note that the "recordsize" is the maximum length of what SIMSCRIPT assumes are variable-length records. Make this number more than three times the number of colums in the model area

open unit 10 for input, recordsize is 2000 ,

file name is "/home/grass/model/tempnap/distprob.asci i" use unit 10 for input

if ropenerr.v $<0$

print 1 line thus

Unable to open distprob.ascii for input

close unit 10

always

for $i=1$ to NROW

do

for $j=1$ to $\mathrm{NCOL}$

do

read BUF using unit 10

Let DISTPROB_INT $(i, j)=$ BUF

loop

loop

close unit 10

3. Relativize raw DISTPROB_IHT values using DISTMAX.

Put relativized values in DISTPROB_REAL.

for $i=1$ to NROW

do

for $j=1$ to NCOL 


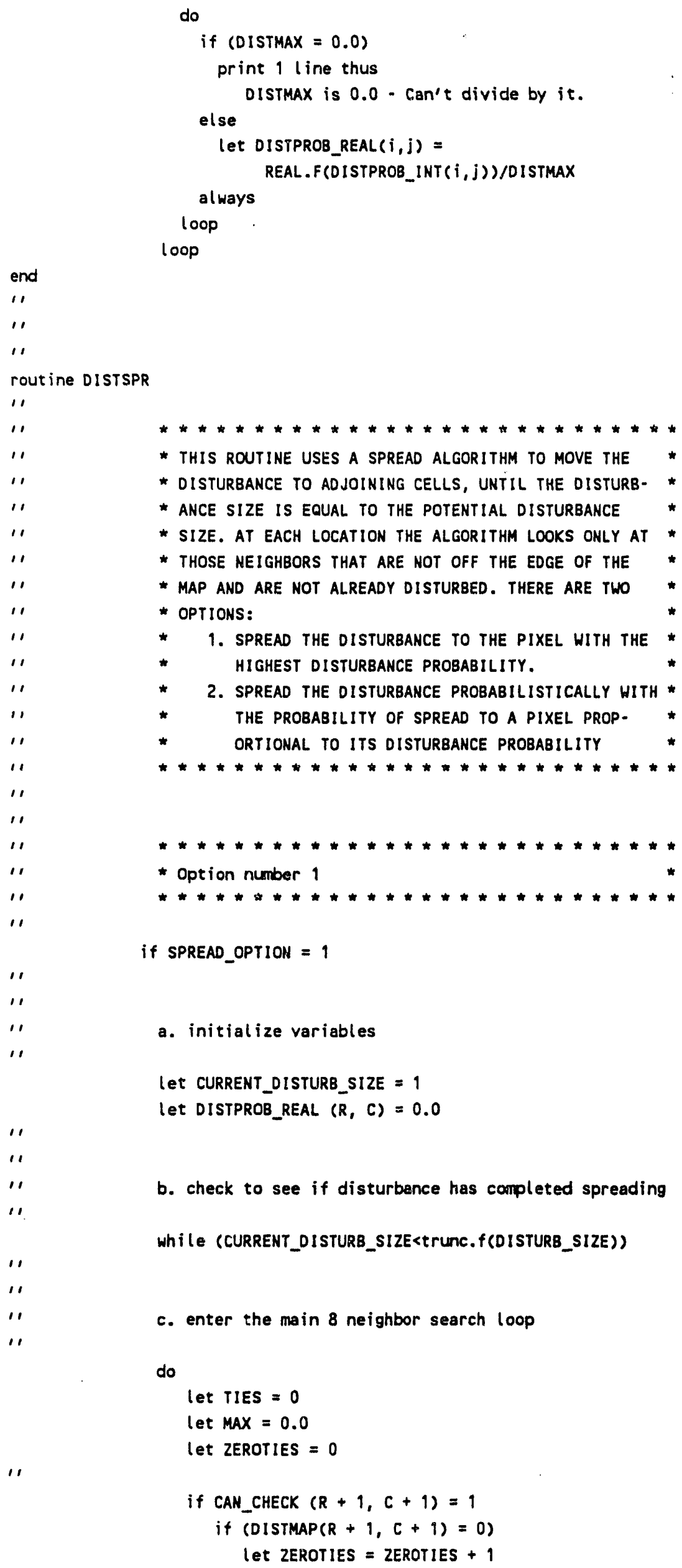

* this routine uses a spread algorithm to move the

* oisturbance to adjoining cells, until the oisturb- *

* ance size is equal to the potential disturbance

* size. at each location the algorithm looks only at *

* those neighbors that are not off the edge of the *

* map and are not already oisturbed. there are two *

- options:

* 1. spread the disturbance to the pixel hith the *

* highest disturbance probability.

* 2. Spread the oisturbance probabilistically with *

* the probabillty of spread to a pixel prop. *

* ortional to its oisturbance probabillity *

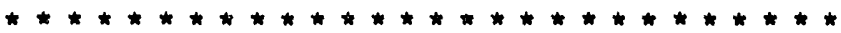

if SPREAD_OPTION $=1$

a. initialize variables

Let CURRENT_DISTURB_SIZE $=1$

let DISTPROB_REAL $(R, C)=0.0$

b. check to see if disturbance has completed spreading

while (CURRENT_DISTURB_SIZE <trunC.f(DISTURB_SIZE))

c. enter the main 8 neighbor search loop

do

Let TIES $=0$

let $\operatorname{MAX}=0.0$

let ZEROTIES $=0$

if CAN_CHECK $(R+1, C+1)=1$

if (DISTMAP(R $+1, C+1)=0)$

let ZEROTIES = ZEROTIES + 1 


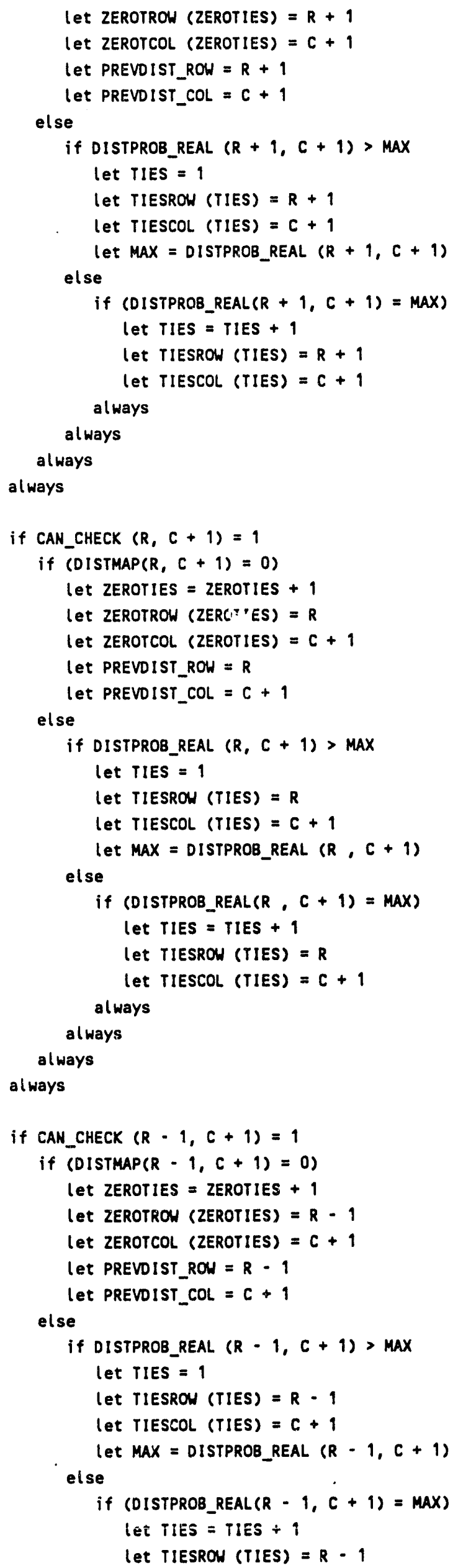



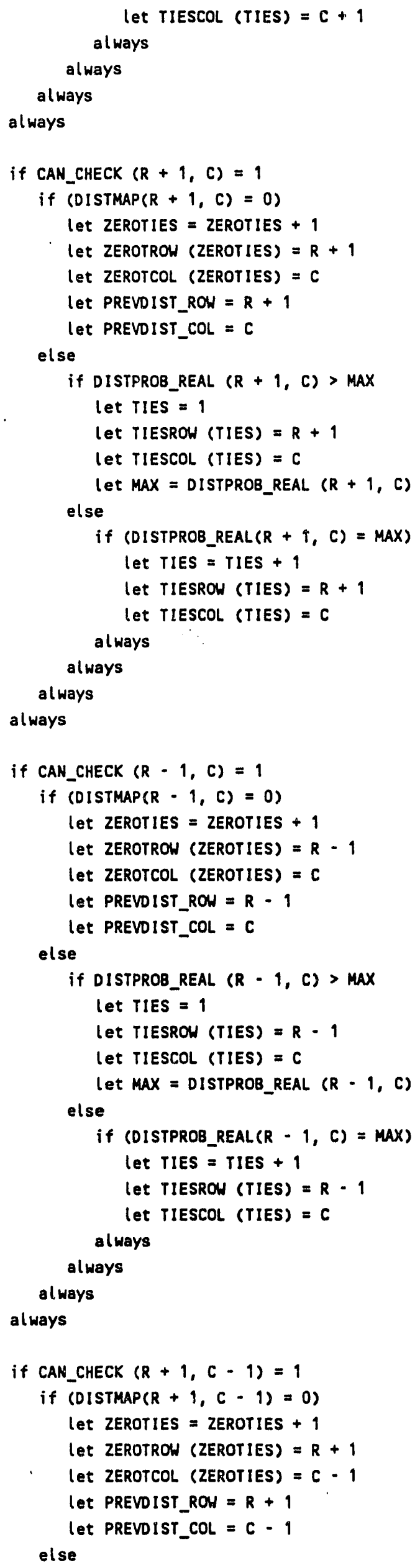


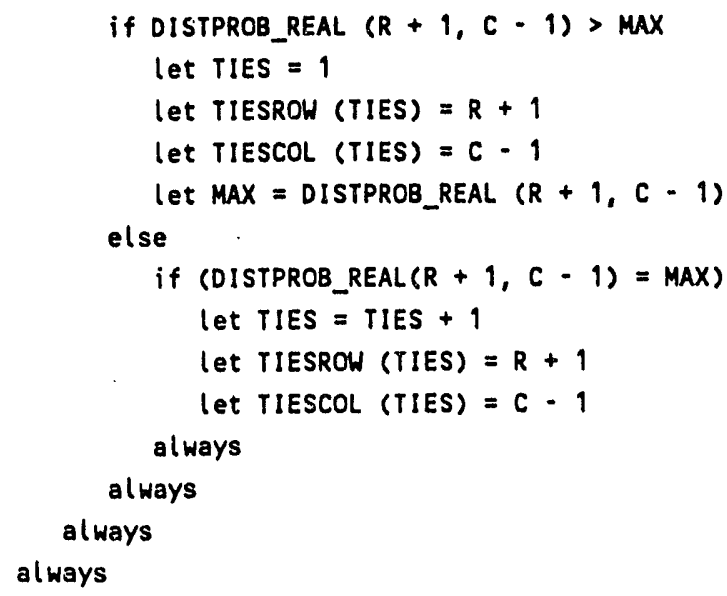


d. If at least one of the 8 neighbors has a disturbance probability $>0.0$

if $\operatorname{MAX}>0.0$

(1) If there is more than one neighboring pixel already disturbed or the disturbance has reached only 1 or 2 pixels, we're not on a "peninsula" or the disturbance has just started. Then find the maximum disturbance probability by randomly choosing among tied values. Disturb that pixel, move to it, and add one to the count of disturbance size.

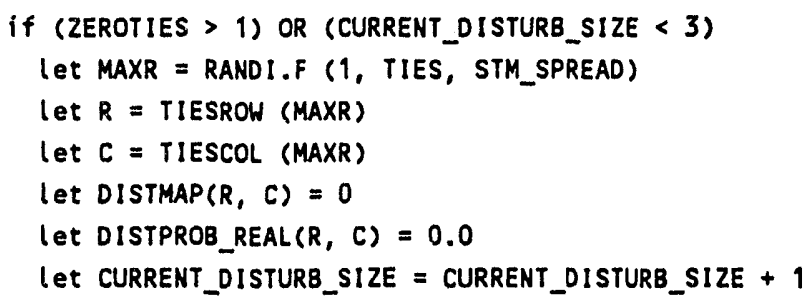

(2) If there is only one neighboring pixel already disturbed and if the disturbance has reached at least 3 pixels, then a "peninsula" could form. To stop this, move back to the already disturbed neighboring pixel and try to spread from there.

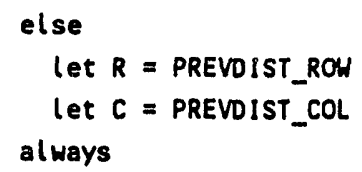

e. If all the neighboring pixels have a disturbance probability of 0.0 , this means that they have all been disturbed al ready or have no probability of being disturbed. Then choose randomly among the 8 neighbors and move to that pixel.

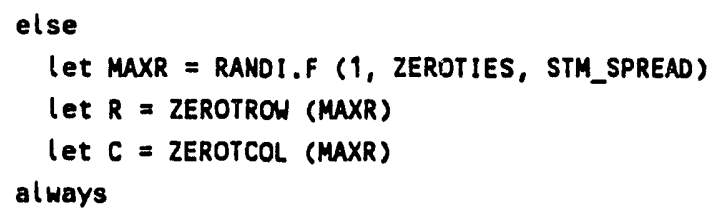


a. initialize variables

let CURRENT_DISTURB_SIZE $=1$

let DISTPROB_REAL $(R, C)=0.0$

b. check to see if disturbance has completed spreading

While (CURRENT_DISTURB_SIZE<trunC.f(DISTURB_SIZE))

c. total up the disturbance probabilities in the eight neighbor cells. first checking to be sure the cell is not off the map and has not already been disturbed. Count up the number of 0.0 probabilities and keep track of where previously disturbed cells are.

do

let ZEROTIES $=0$

let PREVDIST_ROW $=0$

let PREVDIST_COL $=0$

let DISTPROB_tOT $=0.0$

if CAN_CHECK $(R-1, C-1)=1$

if (DISTMAP $(R-1, C-1)=1)$

let DISTPROB_TOT $=$ DISTPROB_REAL $(R-1, C-1)+$ DISTPROB_TOT

let DISTPROB_CUM $(R-1, C-1)=0.0$

let OISTPROB_CUM $(R-1, C-1)=$ DISTPROB_TOT

else

let DISTPROB_CUM $(R-1, C-1)=0.0$

let ZEROTIES = ZEROTIES + 1

let ZEROTROW (ZEROTIES) $=\mathrm{R}-1$

let ZEROTCOL (ZEROTIES) $=\mathrm{C}-1$

let PREVDIST_ROW $=\mathrm{R}-1$

let PREVDIST_COL $=\mathrm{C}-1$

always

al ways

if CAN_CHECK $(R-1, C)=1$

if $(D$ ISTMAP $(R-1, C)=1)$

let DISTPROB_TOT $=$ DISTPROB_REAL $(R-1, C)+$ DISTPROB_TOT

let DISTPROB_CUM $(R-1, C)=0.0$

let DISTPROB_CUM $(R-1, C)=$ DISTPROB_TOT

else

Let DISTPROB_CUM $(R-1, C)=0.0$

let ZEROTIES = ZEROTIES + 1

let ZEROTROW (ZEROTIES) $=\mathrm{R}-1$

let ZEROTCOL (ZEROTIES) $=\mathrm{C}$

let PREVDIST_RON $=R-1$

let PREVOIST_COL $=\mathrm{C}$

always

always

if CAN_CHECK $(R-1, C+1)=1$

if $(D I S T M A P(R-1, C+1)=1)$

let DISTPROB_TOT $=$ DISTPROB_REAL $(R-1, C+1)+$ DISTPROB_TOT

let DISTPROB_CUM $(R-1, C+1)=0.0$ 


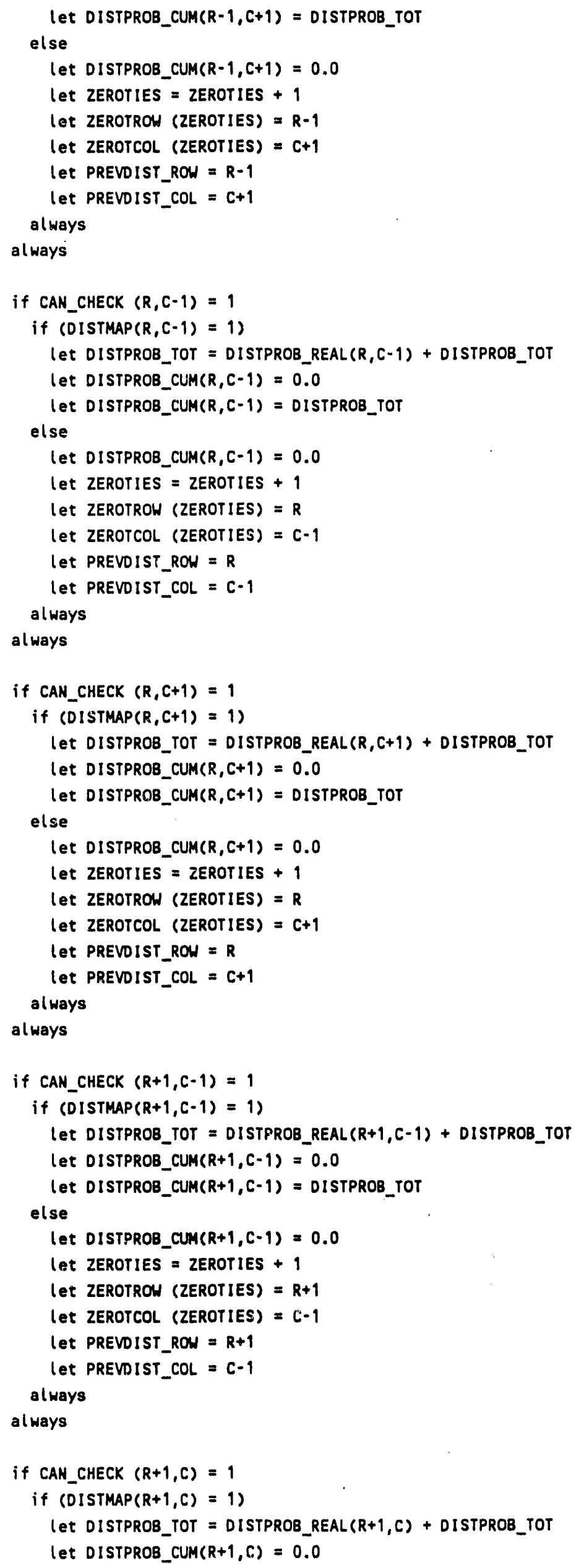




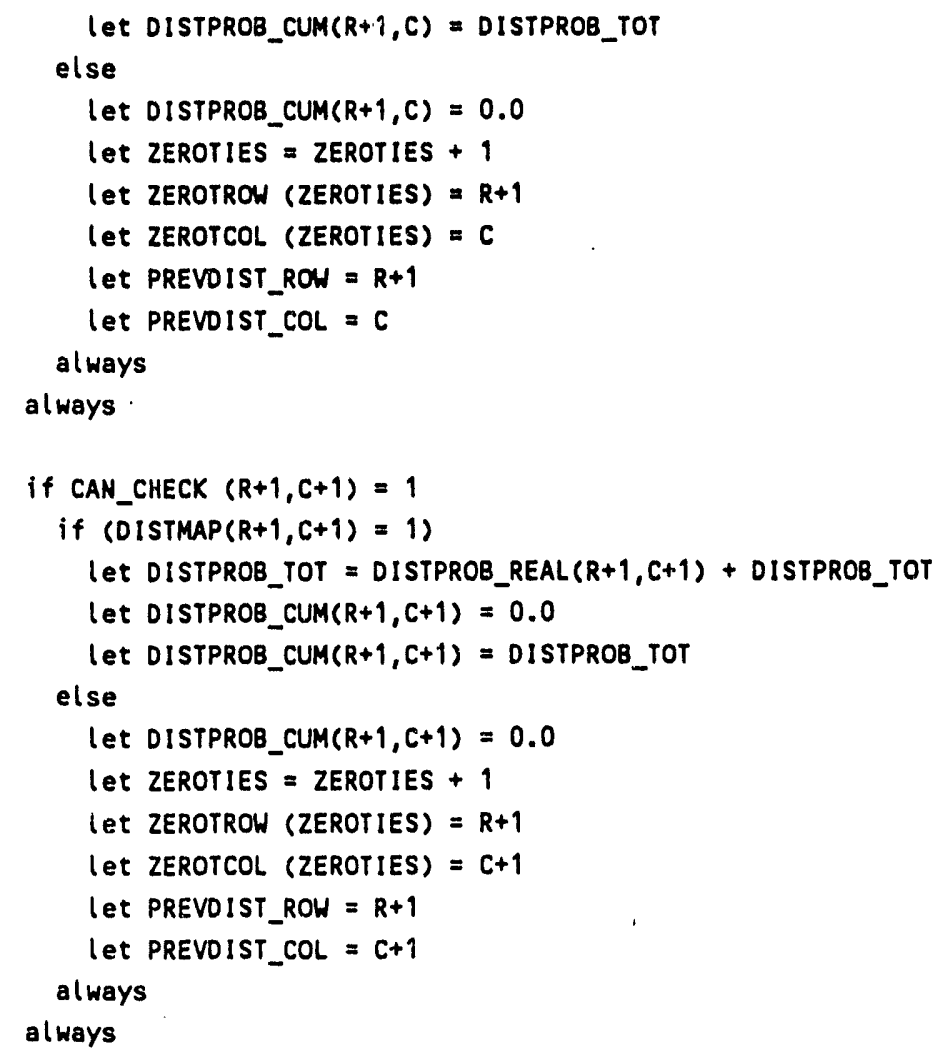

d. If at least one of the 8 neighbors has a disturbance probability $>0.0$

if DISTPROB_TOT > 0.0

(1) If there is more than one neighboring pixel al ready disturted or the disturbance has reached only 1 or 2 pixels, we're not on a "peninsula" or the disturbance has just started. Then select a random number between 0.0 and the total of the disturbance probabilities in the eight neighbor cells. Then find the cell among the eight neighbors whose cumulative probability corresponds to the value of the selected random number. Disturb that pixel, move to it, and add one to the count of disturbance size.

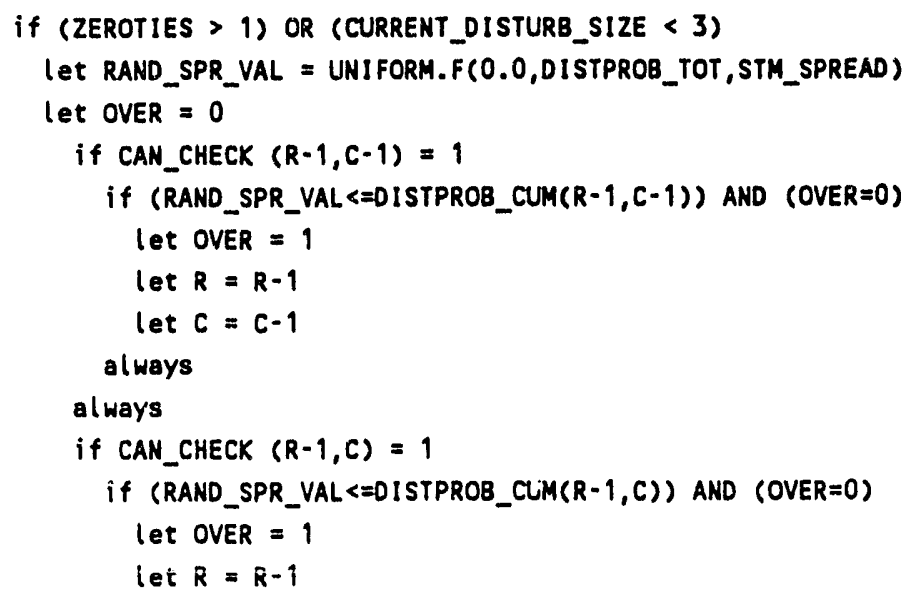




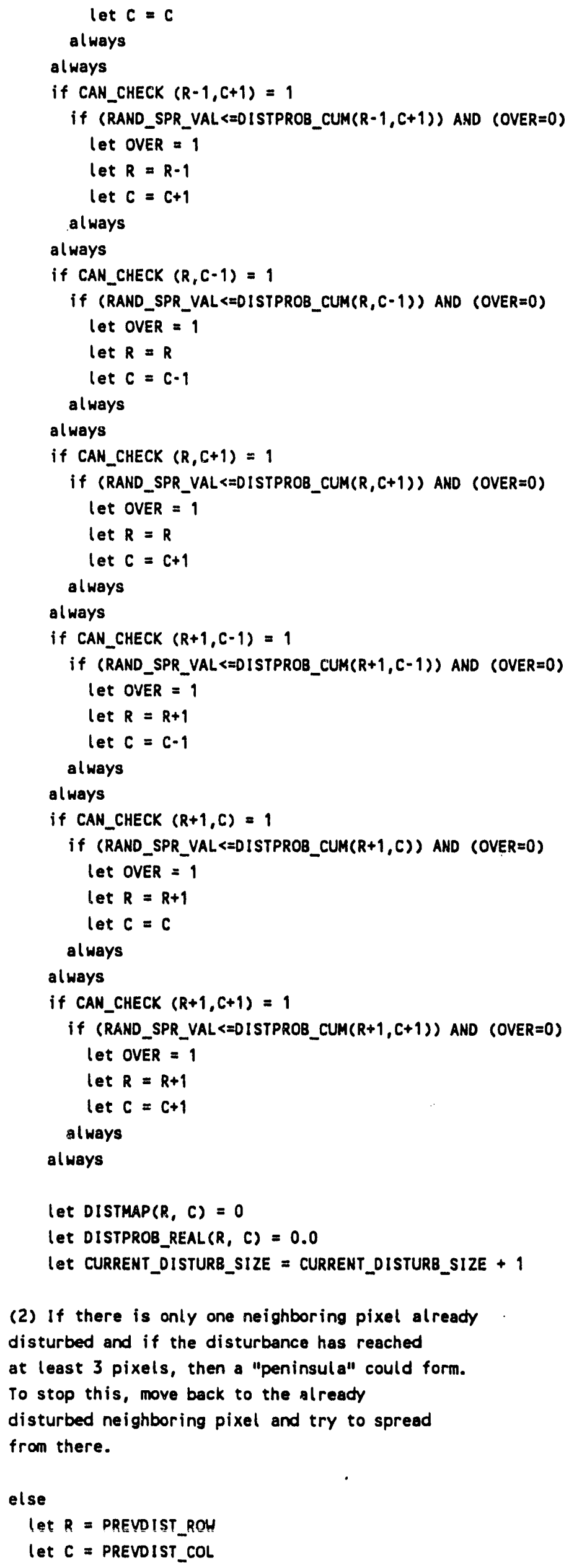

(2) If there is only one neighboring pixel already disturbed and if the disturbance has reached at least 3 pixels, then a "peninsula" could form.

To stop this, move back to the already disturbed neighboring pixel and try to spread from there.

let R $=$ PREVDIST_ROH

let $C=$ PREVDIST_COL

else 


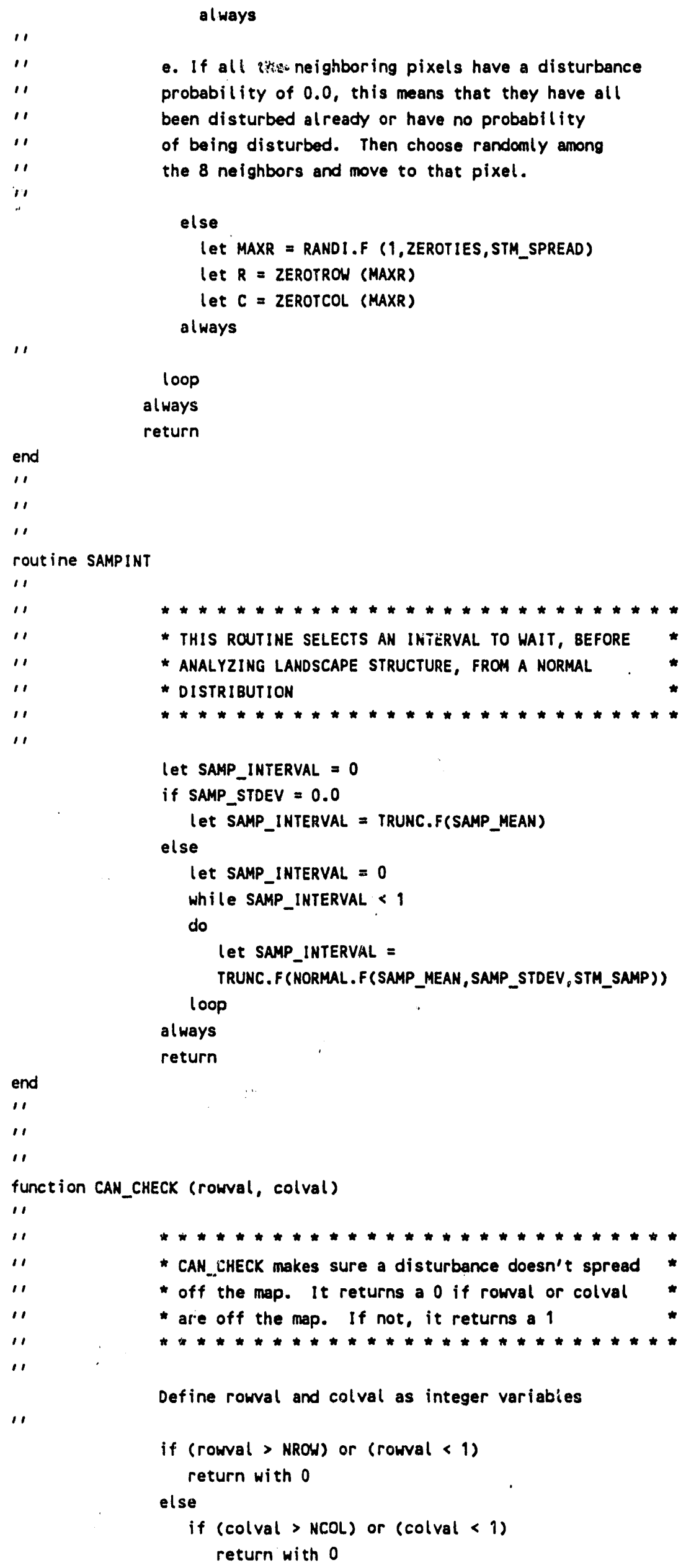




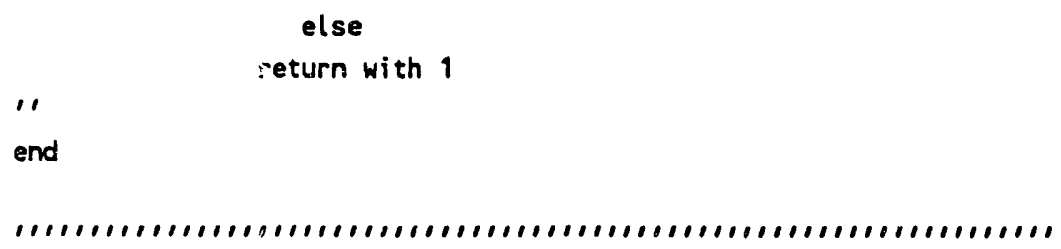


$1 *$ model_lib.c contains a set of C functions that act as independent */

/* library modules to be called by model.sim. model_lib.c has no \#/

$I^{*}$ "main" function, therefore, and will only produce an object module, */

$1^{*}$ not an executable program. Note that all of the functions that $* /$

$7^{*}$ contain GRASS library calls begin with a call to G_gisinit - this is */

$1^{*}$ essential in all independent functions that access GRASS library */

$I^{*}$ functions (the program crashes without $i t$ ).

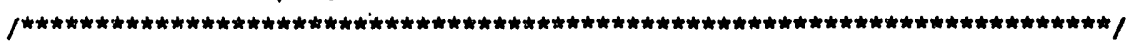

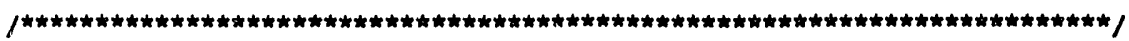

/* C include files */

\#include "stdio.h"

\#include "stdl ib.h"

\#include "math.h"

/* GRASS include files */

\#include "gis.h"

\#include "dig_defines.h"

\#include "dig_structs.h"

$/$ function declarations */

void annual_updt();

void initlayer();

void initdistmap();

void make_distprob();

void putnum();

void reclass_map();

void save_grass_layer( );

void screenputnum();

void show_map();

void sstruct();

void update_map();

void Hrite_ascii();

void write_distprob_ascii(s);

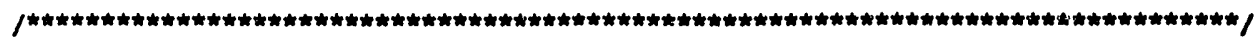

$f^{*}$ annual_updt uses the GRASS r.mapcalc program to update the AGE layer at the $\star /$

$f^{*}$ end of a year. All cells in the age layer are increased by 1.

/* Variables: none \#/

/ Called by: model.sim (simscript) */

/* Calls: G_gisinit, system. \#/

/* Note: The age layer name is assumed to be a constant ("AGE_SCA"). \#/

/ /

void annual_updt()

c

char cond[120];

static char prog_name[] = "annual_updt";

G_gisinit(prog_name);

system("r.mapcalc tmp = 'tmp + 1 'u);

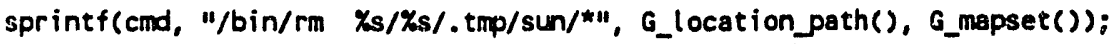

system(cmd); 


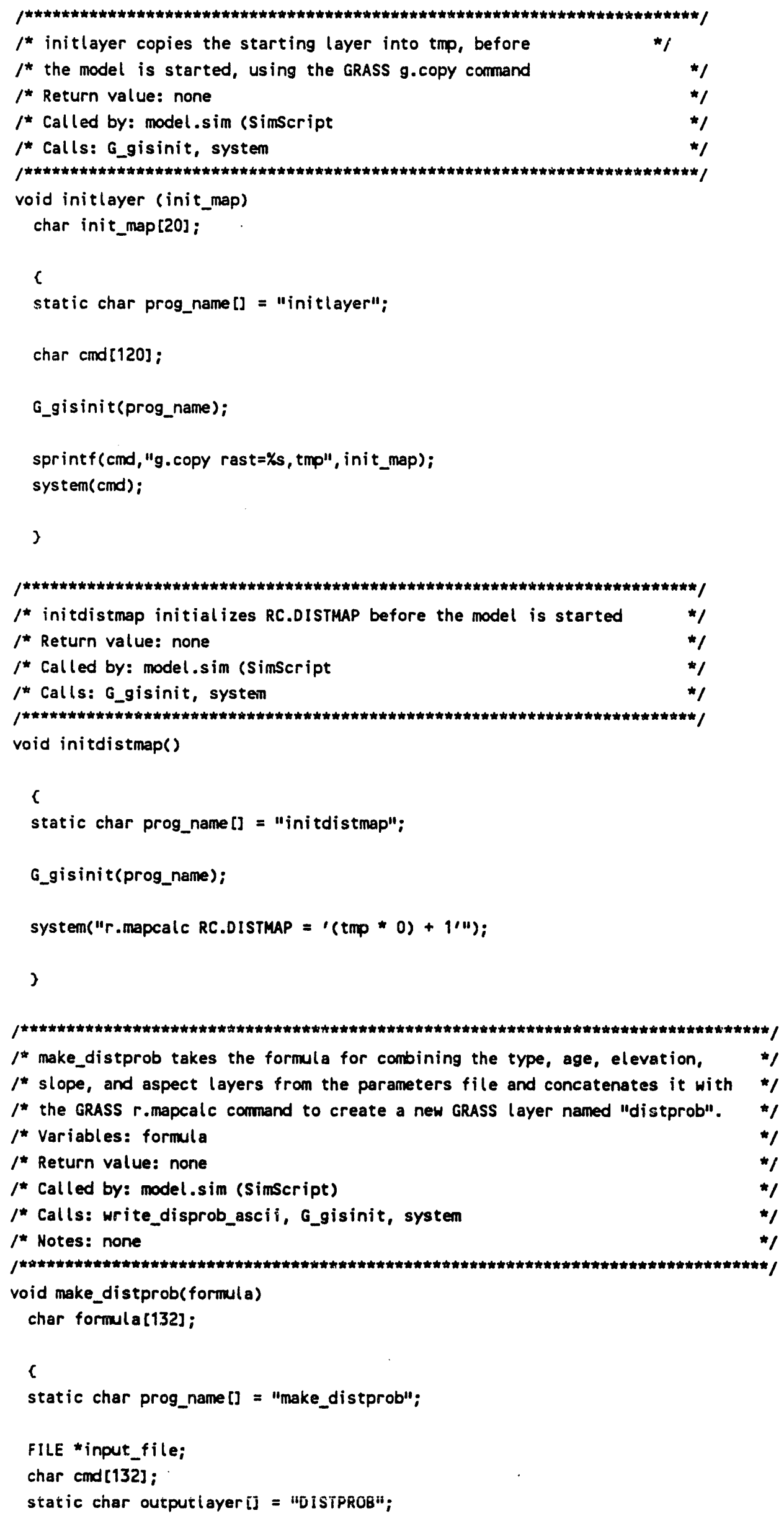




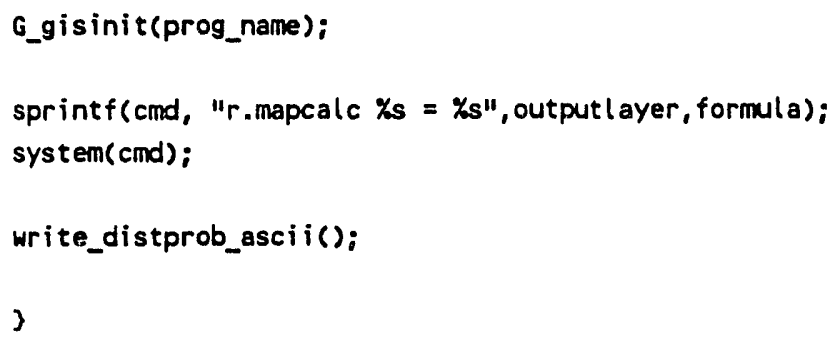




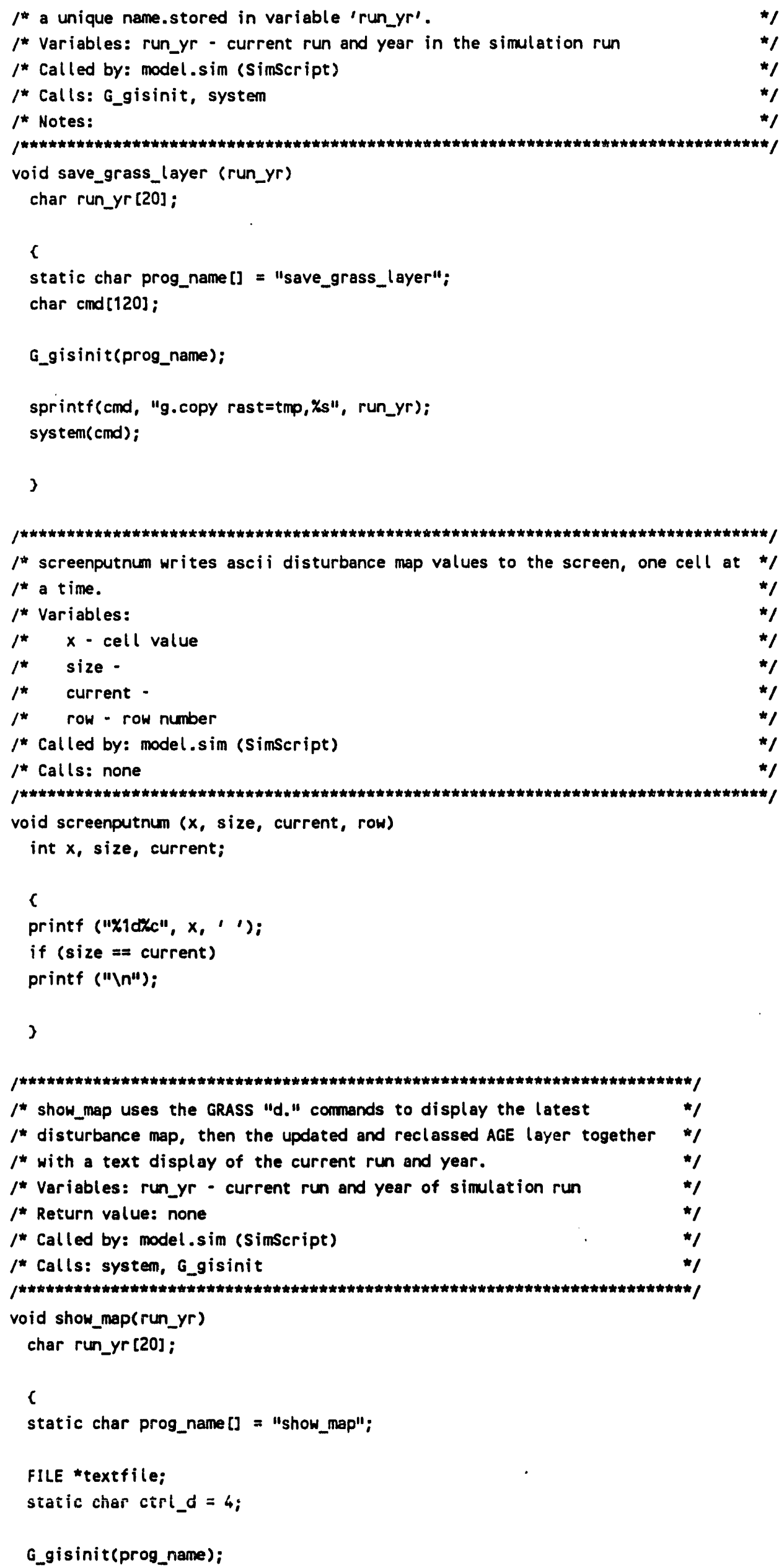




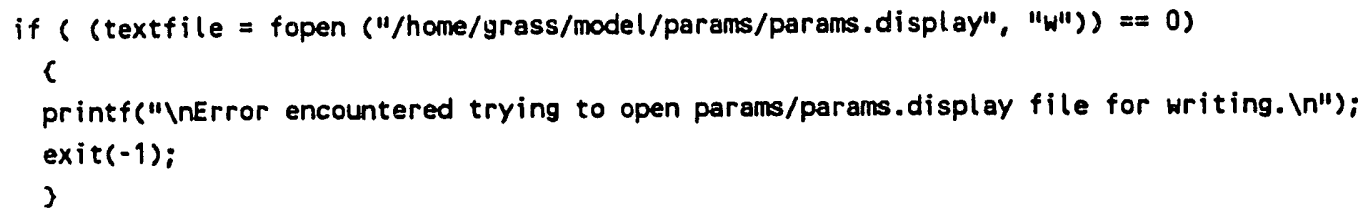
$I^{*}$ see GRASS d.text and d.title documentation for notes on text file format */

fprintf(textfile, ".c yellowin");

fprintf(textfile, ".s 40 (n");

fprintf(textfile, ".8 $\left.1 \backslash n^{\prime \prime}\right)$;

fprintf(textfile," simulation year: Ks $\backslash n^{\prime \prime}$, run_yr);

fprintf(textfile, "\%c", ctrl_d); $/{ }^{\star}$ signals end of text file for d.text */

fclose (textfile);
$I^{*}$ display the disturbance map, then the updated age map and text */

system("d. colormode mode=fixed");

system("d.frame - e");

system("d.frame -c frame=map1 at $=15,100,15,100$ ");

system("d.frame -c frame=msg at $=0,10,0,100 "$ );

system("d. frame - $c$ frame=map2 at $=85,100,85,100$ ");

system("d. frame -s frame=map1");

system("cat /home/grass/model/params/rules.file | r.colors map=map.recl color=rules");

system("d. rast map=map. recl");

system("d.frame -s frame=map2");

system("cat /home/grass/model/params/rules.file | r.colors map=RC.DISTMAP color=rules");

system("d. rast map=RC.DISTMAP");

system("d.frame -s frame=msg");

system("d.text < /home/grass/model/params/parans.display");

system("d.frame -c frame=leg at=15, 100,0,13");

system("d. frame -s frame=leg");

system("d. legend map=map. $\operatorname{rec}(")$;

3

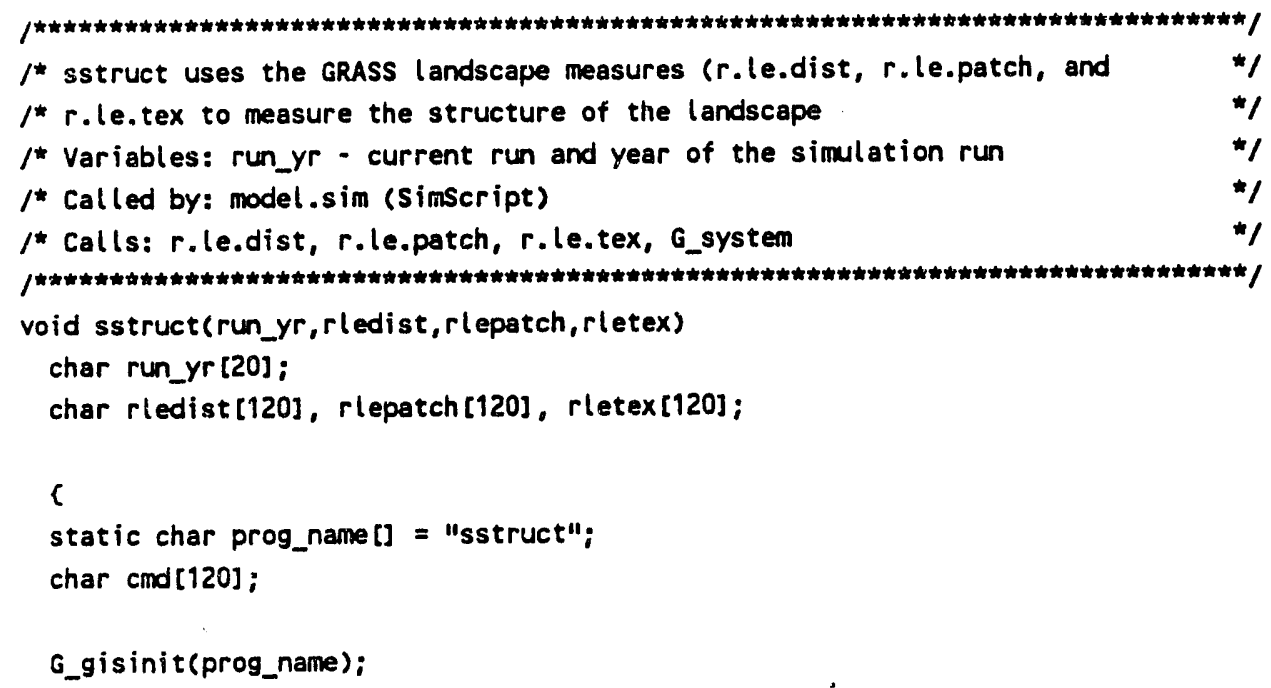




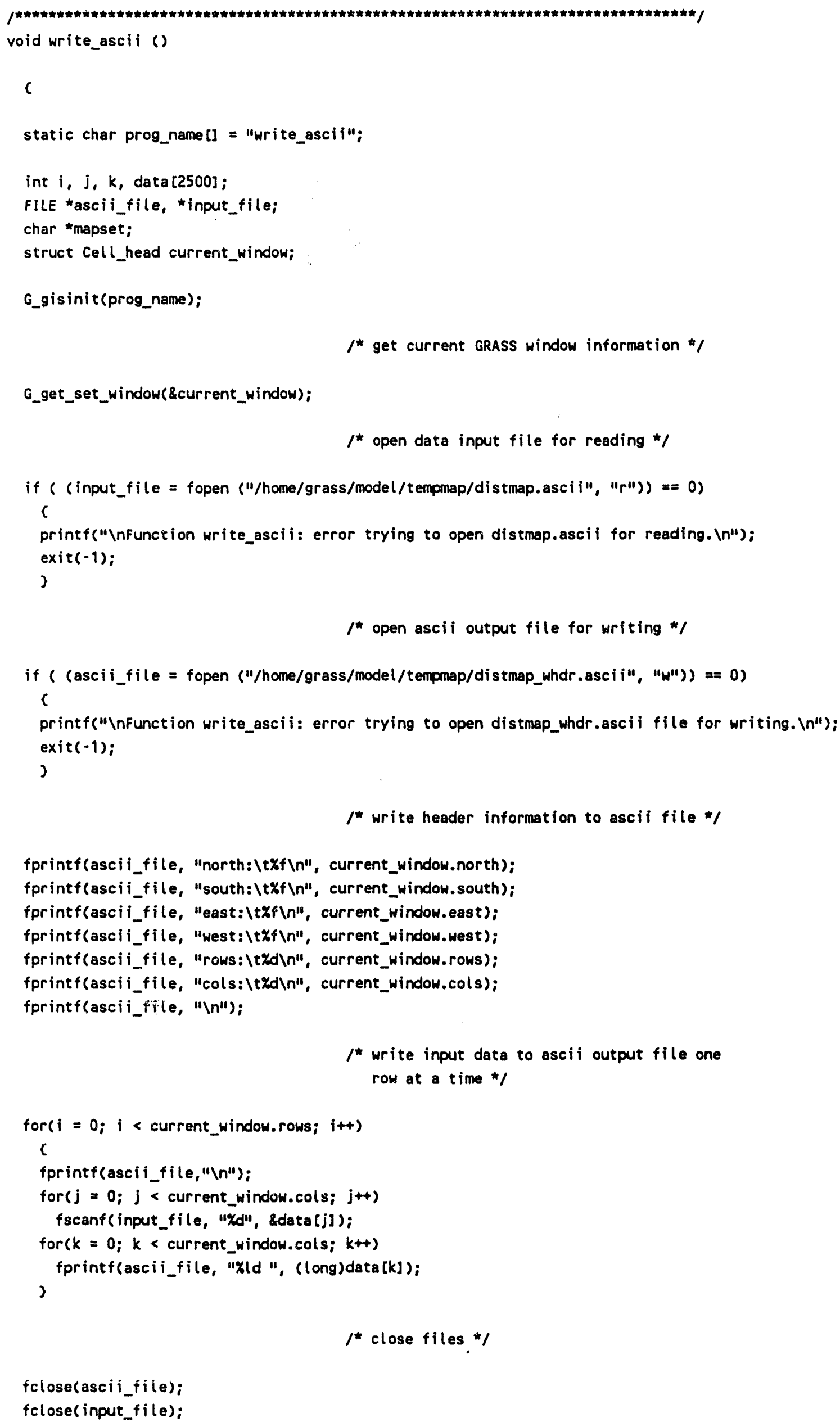




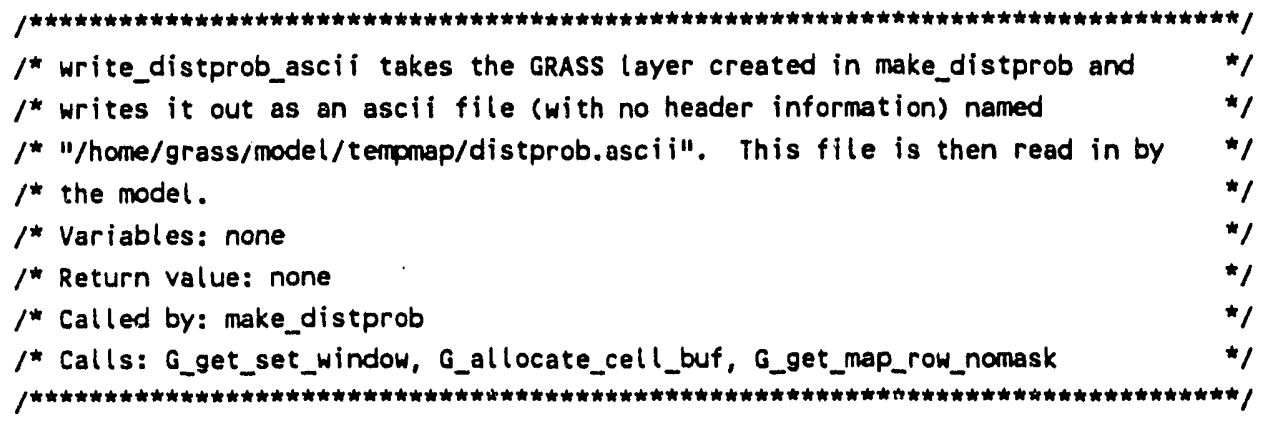
void write_distprob_ascii()

$c$

int $i, j, f d$, row;

char *mapset;

static char filename [] = "DISTPROB";

CELL *cell;

FILE *ascii_file;

struct Cell_head now_window;

/* get current window information */

G_get_set_window( \&now_window);

/* locate and open distprob GRASS layer for reading */

if $\left(\right.$ imapset $=G_{-} f$ ind_cel $($ (filename, $"$ 'I') $)==$ NULL $)$

$c$

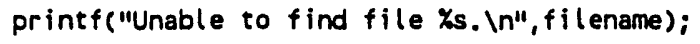

exit(1);

3

if $\left(f d=G_{-}\right.$open_cell_old(fil ename, mapset $\left.\left.)\right)<0\right)$

exit $(-1) ; / /^{\star}$ p.82 $*$

$I^{\star}$ open ascii output file for writing */

if $($ (ascii_file $=$ fopen ("/home/grass/model/tempmap/distprob.ascii", "w")) $=0$ )

C

printf("InError encountered trying to open tempmap/distprob.ascii file for writing. In"); exit(-1);

3

$1^{\text {* }}$ allocate memory for reading a row of the DISTPROB map $* /$

cell = G_allocate_cell_buf();

/t get DISTPROB rows one at a time and then print contents to ascii file $\star /$

for $(i=0 ; i<$ now_window.rows; $i++)$

$c$

G_get_map_row_nomask (id, cell, i ); 
for $(j=0 ; j<$ now_window.cols; $j++)$

fprintf(ascii_file, "X1ld $x_{c} "$, (long)cell[j], ' ');

fprintf (ascii_file, "\n");

)

$I^{*}$ close up the files when done $* /$

G_close_cell( $f d)$;

fclose(ascii_file);

了 
APPENDIX D. DOCUMENTATION FOR THE R.LE PROGRAMS 


\title{
The r.le Programs
}

A set of GRASS programs

for the quantitative analysis of landscape structure

Version 2.0

January 1, 1993

\author{
William L. Baker, Principal Investigator \\ Yunming Cai, Research Assistant \\ Saleh Musleh, Research Assistant \\ Jerry Bucher, Research Assistant \\ Department of Geography and Recreation \\ University of Wyoming \\ Laramie, Wyoming 82071
}

This set of programs was developed in its entirety with funds from U.S.

Department of Energy Grants DE-FG02-89ER60883 and DE-FG02-90ER60977.

This support does not constitute an endorsement by DOE of the views expressed in this document. 


\section{TABLE OF CONTENTS}

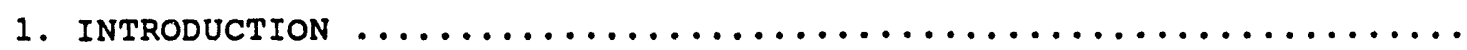

1.1. Purpose of the $r$. le programs .....................

1.2. Relationship of the $r .1$ e programs and GRASs .......... 3

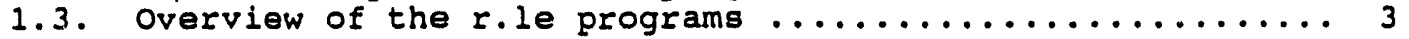

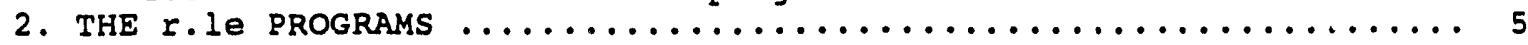

2.1. Operation ................................ 5

2.2. Data input .............................. 5

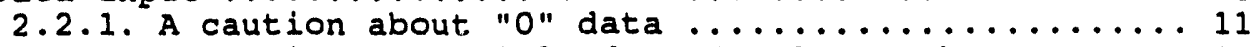

2.2 .2 . A caution about islands and $r .1 e . p a t c h \ldots \ldots . . . .11$

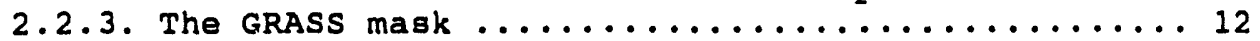

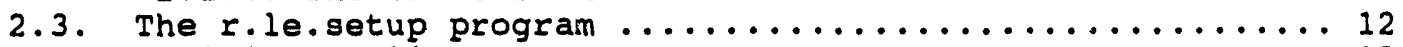

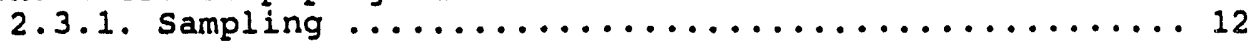

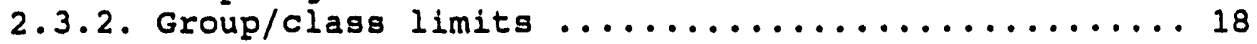

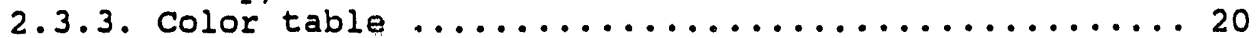

2.4. Syntax for the $r .1$ e analysis programs .............. 21

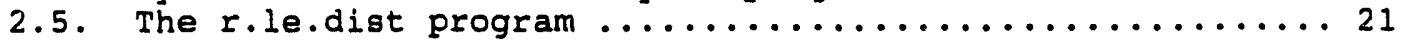

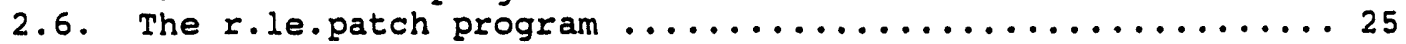

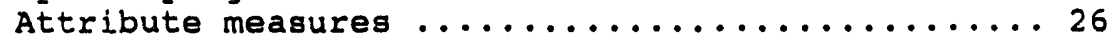

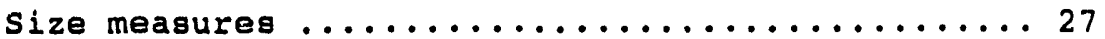

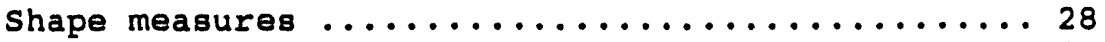

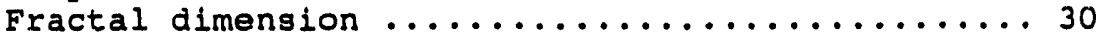

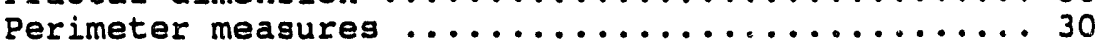

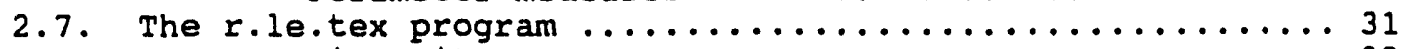
Diversity measures .................... 32

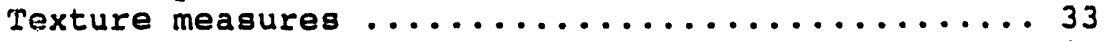

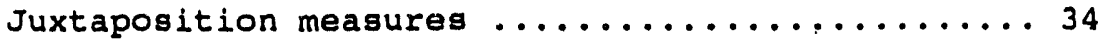

Edge measures ......................... 37

2.8. Output from the r.le analysis programs ............. 37

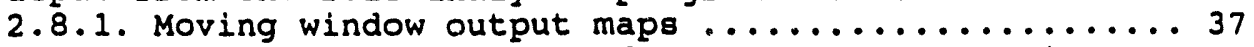

2.8.2. Output files and data formats from non-moving

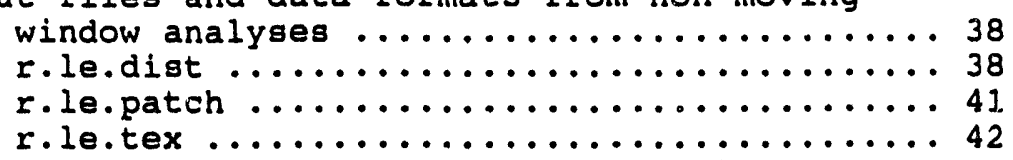

2.8.3. Data analysis with external statistical and graphics software ................ 42

2.9. The r.le.null program ........................ 43

2.10. The r.le.rename program ..................... 43

2.11. The r.le.show program ....................... 44

2.12. The r.le.trace program ......................... 45

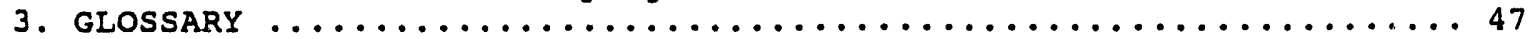

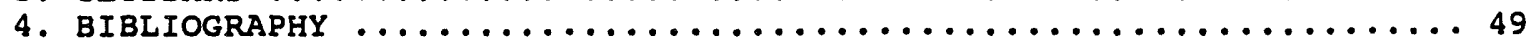

5. APPENDICES ..................................... 52

1. Limits ......................................... 53

2. Time needed to complete analyses with the r.le programs .... 54

3. Help menus for the $r$. le programs ................... 55 


\section{INTRODUCTION}

\subsection{Purpose of the r.le programs}

Since the 1970s, with the availability of satellite data, there has been an increasing interest in the structure of the earth on the scale of kilometers or hundreds of kilometers. Landscape ecology is a multi-disciplinary pursuit, involving geographers, biologists, sociologists, remote sensors, and many others. The focus of landscape ecology is on the dynamics and structure of the biosphere, including human activities, on the scale of kilometers (Risser et al. 1984; Forman and Godron 1986; Urban et al. 1987). The science of landscape ecology expanded rapidly in the 1980s, and methods for the quantitative analysis of landscape structure also were developed (e.g. Mead et al. 1981; Gardner et al. 1987; Krummel et al. 1987; Milne 1988; Griffiths and Wooding 1988), yet there is no generally available software for the quantitative analysis of landscape structure that will work within a geographical information system (GIS).

The r.le programs have been designed to provide software for calculating a variety of common quantitative measures of landscape structure. The programs were specifically designed for analyzing the structure of landscapes output from a simulation model, but can be used to analyze the structure of nearly any landscape.

\subsection{Relationship of the r.le programs and GRASS}

The r.le programs are intended to be part of the Geographical Resources Analysis Support System (GRASS), a public-domain geographical information system (GIS), designed and developed through the Environmental Division of the U.S. Army Construction Engineering Research Laboratory in Champaign, Illinois (USA-CERL 1991) (Fig. 1). GRASS is primarily a raster-based GIS, but now has extensive vector handling capabilities. GRASS requires the UNIX operating system, but can be used on a variety of workstations, as well as 80386 -class personal computers. The programs use GRASS version 4.0 and its XDRIVER.

The r.le programs directly use the GRASS database, GRASS libraries, and the GRASS data structure (Fig. 1) in the calculation of measures of landscape structure, and use GRASS for the entry of digitized data. GRASS also provides a number of separate image processing, data manipulation, and mapping programs which can be useful for preparing data for analysis with the r.le programs and for displaying output.

\subsection{Overview of the r.le programs}

The r.le programs are designed for analyzing landscapes composed of a mosaic of patches, but, more generally, these programs are capable of analyzing any two-dimensional raster or array whose entries are integer values. The r.le programs have options for controlling the shape, size, number, and distribution of 
Figure 1

\section{GRASS and r.le Programs}

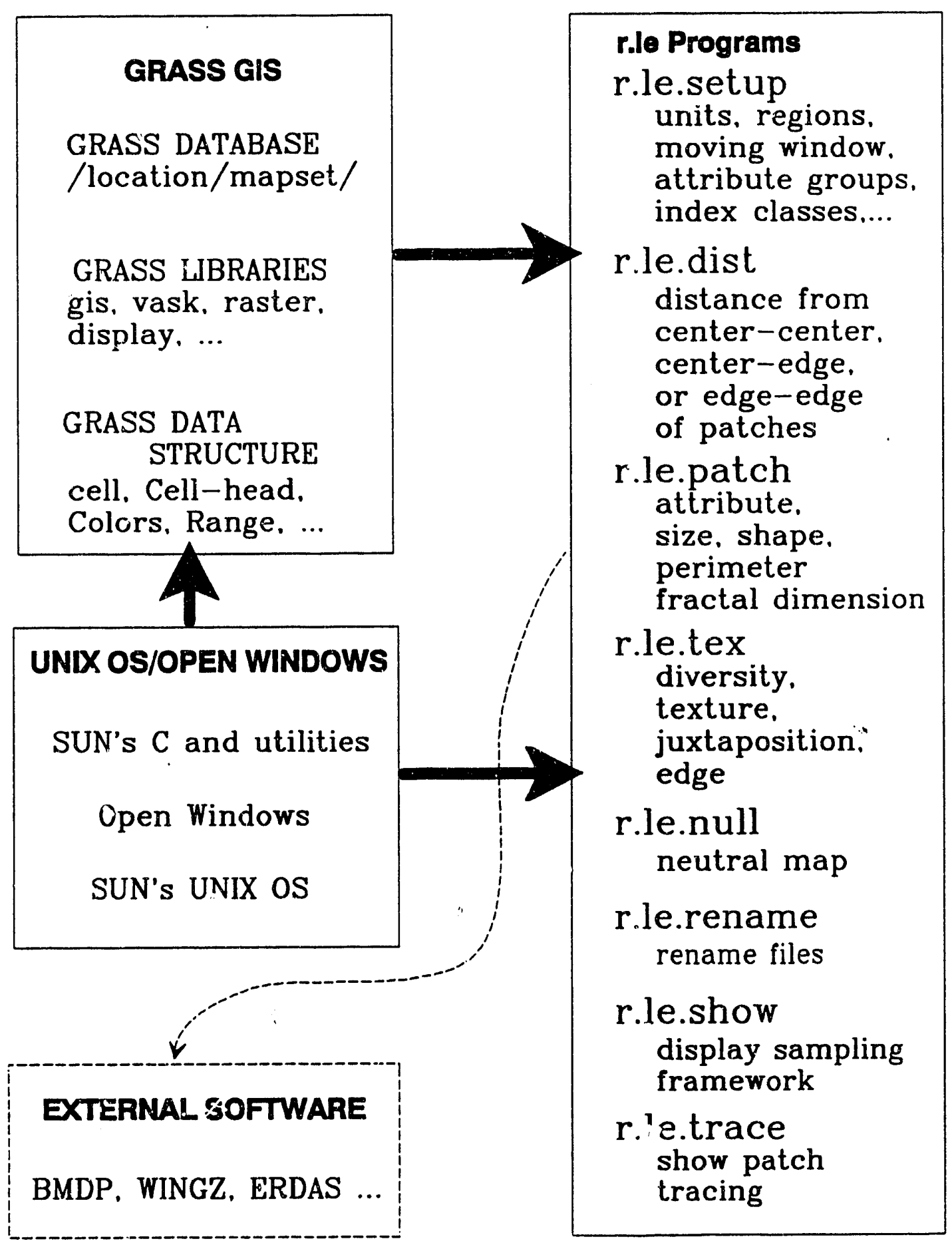


sampling areas used to collect information about the landscape. Sampling area shapes can be square, or rectangular with any length/width ratio. The size of sampling areas can be changed, so that the landscape can be analyzed at a variety of spatial scales. Sampling areas may be distributed across the landscape in a random, systematic, or stratified-random manner, or as a moving window.

The r.le programs can calculate a number of measures that produce single values as output (e.g. mean patch size in the sampling area), as well as measures that produce a distribution of values as output (e.g. frequency distribution of patch sizes in the sampling area) (Table 1). The programs include no options for graphing or statistically analyzing the results of the analyses. External software must be used.

The programs were developed on a SUN SPARCstation 1 workstation running the Sun OS 4.1.2 operating system and the Open Windows 3.0 windowing programs (Fig. 1). The code was written in the $C$ programming language and makes use of functions provided in the GRASS programmers' library (Shapiro et al. 1989).

\section{THE r.le PROGRAMS}

\subsection{Operation}

To run the r.le programs, the user must first start GRASS (type "grass4.0") and set up the working environment in GRASS by specifying the GRASS location and map layers to be used. The sequence of operations usually is to first use r.le.setup to set up the sampling framework (e.g., regions, sampling unit size and shape, etc.) and then use the other r.le programs (e.g. r.le.tex, r.le.patch, r.le.dist) to make the desired measurements (Fig. 2). All of the r.le programs operate from the GRASS command prompt $(>)$. The commands and their parameters are entered after the GRASS command prompt, and the programs then go through a sequence of operations to complete the setup and measurements (Fig. 3). Output from r.le.setup goes in the subdirectory "r.le.para" while output from the other r.le programs goes in the subdirectory "r.le.out". These subdirectories are created automatically when the programs are invoked, and are made subdirectories within the directory from which the programs are invoked.

\subsection{Data input}

The r.le programs work directly with map layers that have been input and preprocessed in GRASS. Data from Landsat Multi-Spectral Scanner (MSS) or Thematic Mapper (TM) can be downloaded into GRASS using the image processing programs in GRASS. GRASS also has programs for reading files produced by ERDAS and ARC/NFO, and for reading ASCII raster files. Vector information can be input using the GRASS digitizing programs. Vector information must be converted to raster data using the GRASS program "v.to.rast" prior to using the r.le 
Figure 2

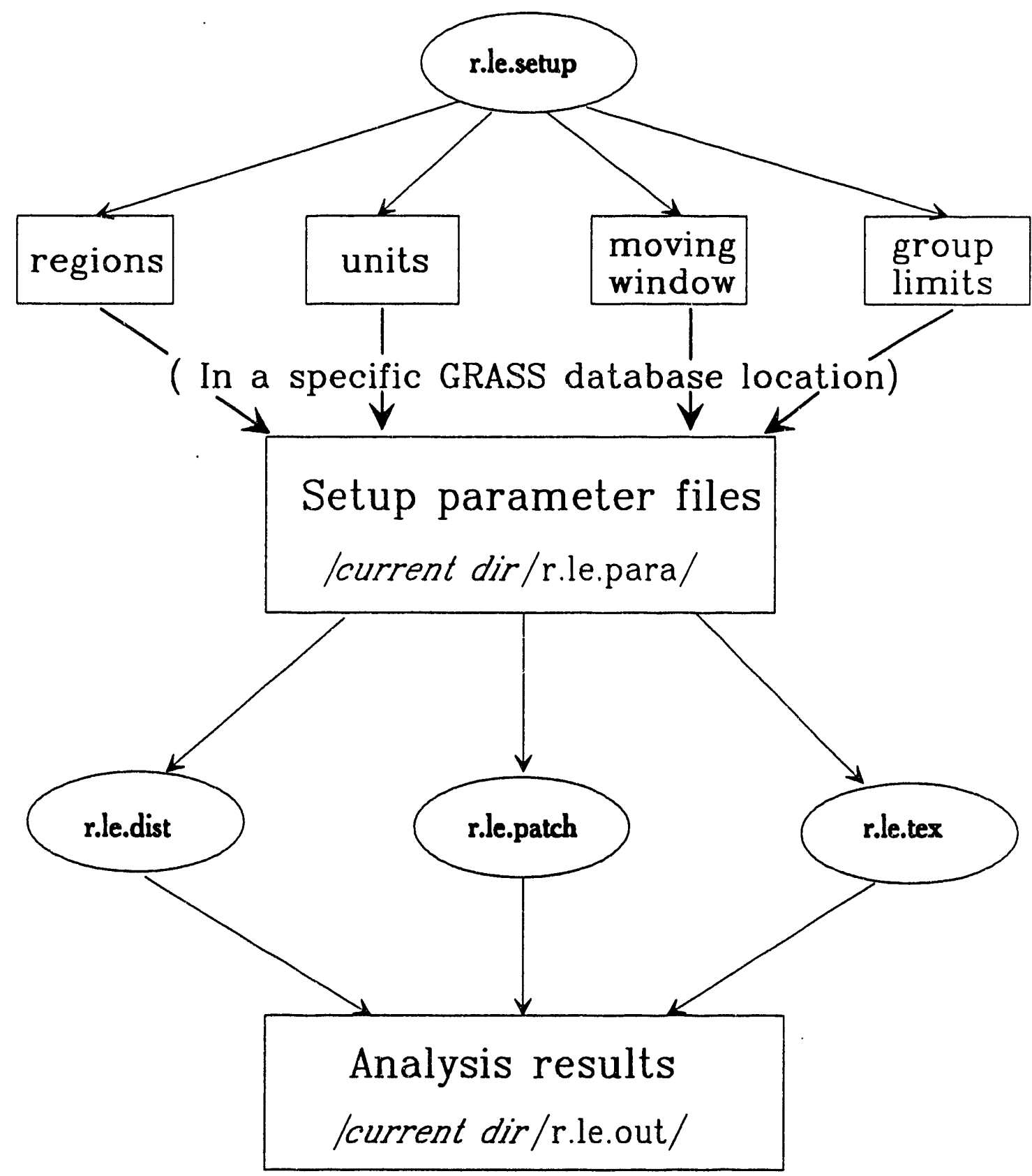


Figure 3

STRUCTURE OF r.le ANALYSIS PROGRAMS

r.le.dist - distance analysis

r.le.patch - patch analysis

r.le.tex - texture analysis

\section{r.le COMMANDS}

r.le.* param1 param2... paramn

SETUP FILES

/current dir/r.le.para/*
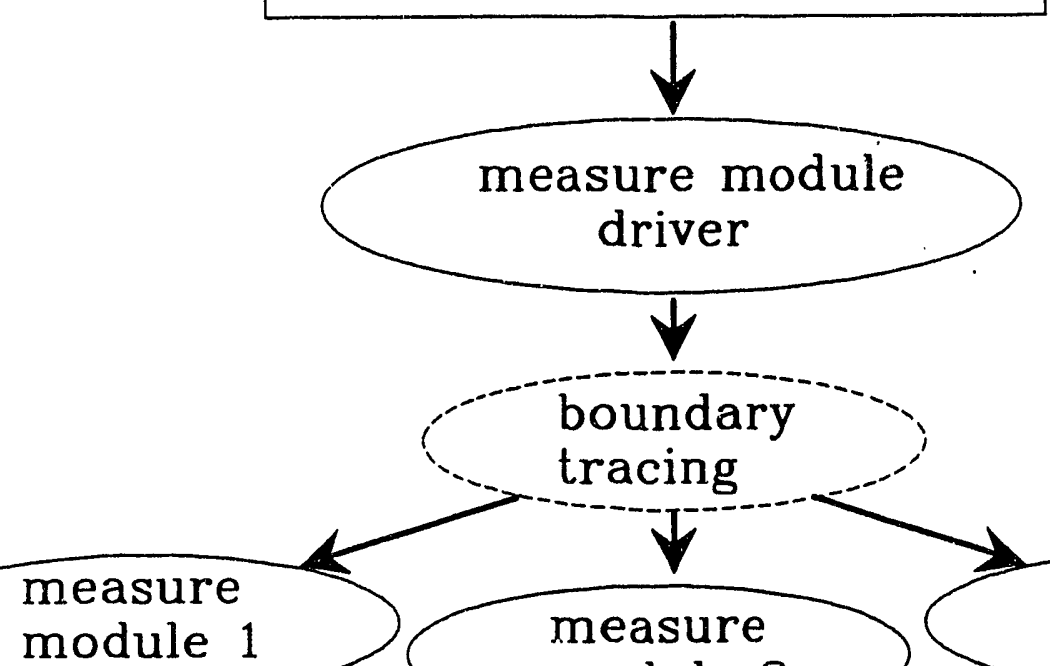

driver 
TABLE 1. Measures that can be calculated by the r.le programs. gp = attribute group, $C C=$ center-to-center distance, $C E=$ center-to-edge distance, $\mathrm{EE}=$ edge-to-edge distance.

r.le.dist

MEASURES:

Mean distance

Standard deviation distance

Mean distance by gp

Standard deviation distance by gp

Number of distances by distance class

Number of distances by distance class by $g p$

METHODS:

Each patch to all adjacent neighbors CC

Each patch to all adjacent neighbors $C E$

Each patch to nearest patch of same gp CC

Each patch to nearest patch of same gp CE

Each patch to nearest patch of same gp EE

Each patch to nearest patch of different gp CC

Each patch to nearest patch of different $g p C E$

Patches of $1 \mathrm{gp}$ to nearest of specific gp CC

Patches of $1 \mathrm{gp}$ to nearest of specific gp CE

Patches of $1 \mathrm{gp}$ to nearest of specific gp EE

r.le.patch

ATTRIBUTE:

Mean pixel attribute

Standard deviation pixel attribute

Mean patch attribute

Standard deviation patch attribute

Cover by gp

Density by gp

SIZE:

Mean patch size

Standard deviation size

Mean patch size by gp

Standard deviation size by gp

Number by size class

Number by size class by gp 
Table 1. Continued.

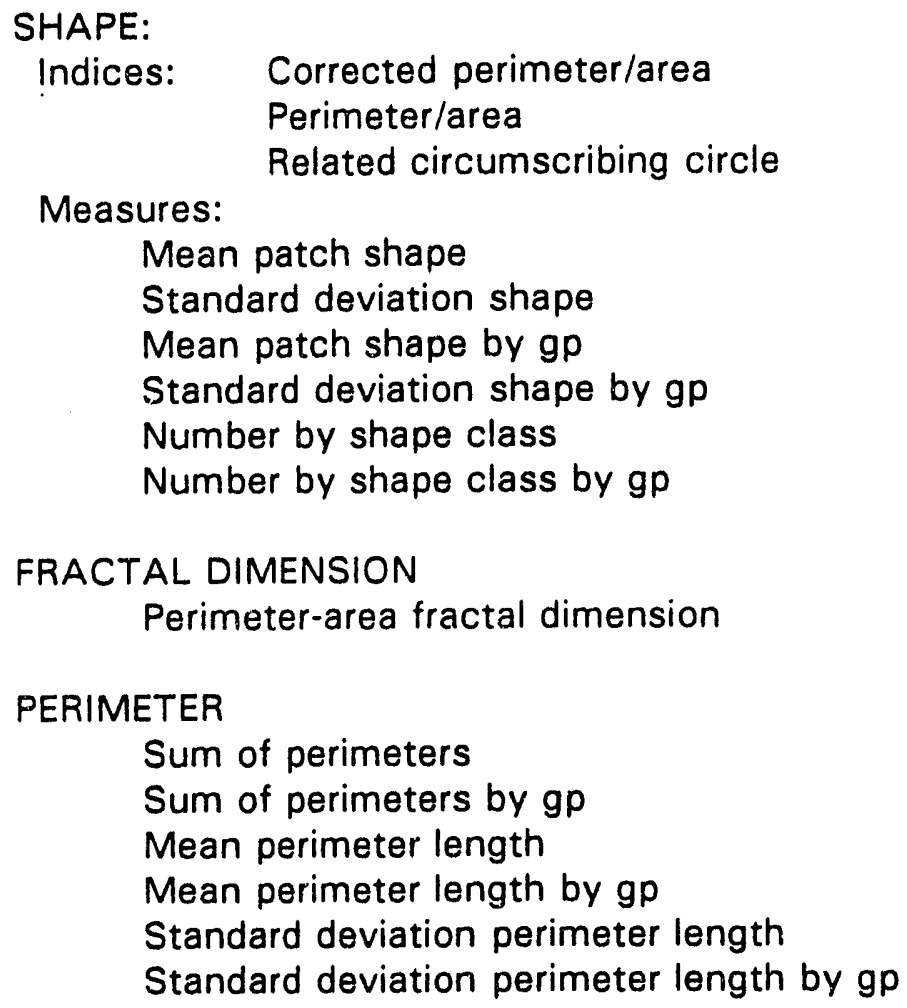

r.le.tex

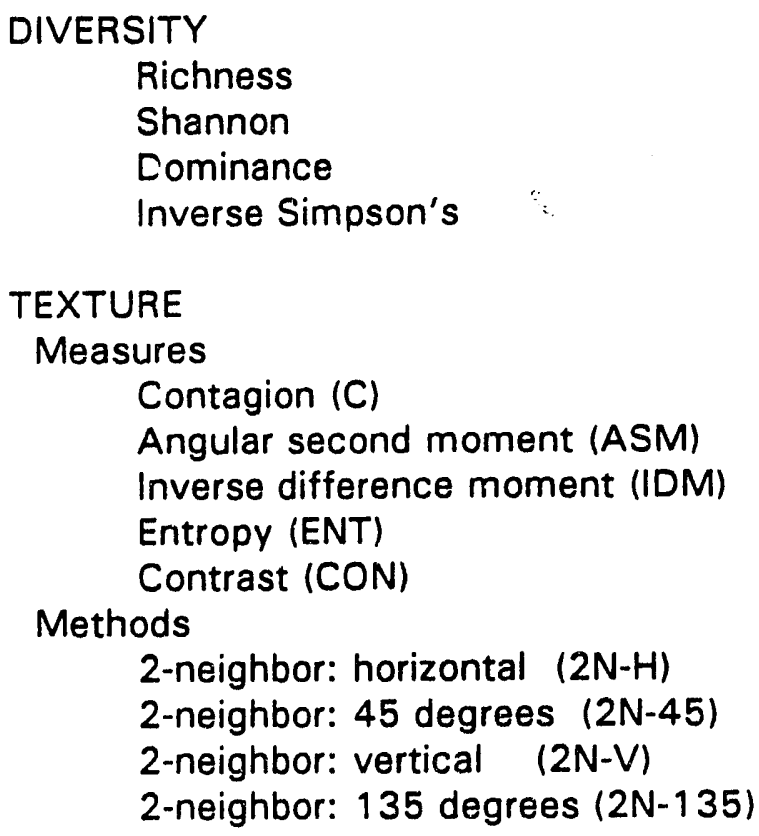


Table 1. Continued.

4-neighbor: horizontal/vertical (4N-HV)

4-neighbor: diagonal (4N-DIAG)

8-neighbor: (8N)

JUXTAPOSITION

Mean juxtaposition

Standard deviation juxtaposition

EDGE

Sum of edges 
programs. Preprocessing capabilities of GRASS include programs to rectify imagery so that it matches a planirnetric map and programs for classifying raw multi-band data.

The r.le programs were conceived for analyzing maps of patches. Any raster map can be considered to contain patchiness and can be analyzed using the programs, but a variety of landscape data can be more specifically considered "patch" data. Patches may be disturbance patches, remnant patches, environmental resource patches, introduced patches, or simply patchy entities on a map (Forman and Godron 1986). Patches may simply be landscape elements (Forman and Godron 1986), such as roads, dwellings, forest patches, grassland patches, hedgerows, or fields. Patches could also be types of forest in a forested landscape (e.g. deciduous forest, recently-burned forest, conifer forest), or types of grassland in a prairie landscape. Patches of different age occur in landscapes subject to disturbances (e.g. fires, floods), where the age of the patch represents the time since it was last disturbed. Patches could also be the types identified by completing a classification of spectral data in a Landsat image, or in a scanned aerial photograph. In general, patches are simply the result of grouping pieces of the landscape into units whose members share a common set of attributes.

\subsubsection{A caution about " 0 " data}

GRASS specifies that the integer " 0 " does not have the traditional meaning of zero. Instead "O" in GRASS means "no data." For this reason the r.le programs do not include " $O$ " values in calculations. Raster pixels that contain " 0 " are excluded from all calculations and are skipped when the moving window is centered over them. If you have a patch that has the attribute " 0 " that you want to have included in the r.le calculations, you must recode it (use r.reclass) to some other attribute value (e.g., -999) prior to using the r.le programs. We hope that in future GRASS releases there will be a provision for the integer zero, which does on occasion have real meaning (e.g., age $=0$ for newly created patches).

\subsubsection{A caution about islands and r.le.patch}

An island is a pixel or set of pixels with attribute $x$ contained wholly within a surrounding patch whose attribute is not $x$. The r.le.tex program does not use patches, and the r.le.dist program does not need to distinguish islands, but the r.le.patch program must trace patches and recognize islands. This is because the size and perimeter of patches will be incorrect unless the islands they contain are identified, and size and perimeter are corrected to account for these islands.

Identifying that a patch is an island and then finding the surrounding patch is very difficult in raster format and is also very time consuming. The user is cautioned that if the map to be analyzed contains many islands then r.le.patch may be very slow.

Moreover, the tracing algorithm currently will only identify one level of 
nesting of islands. If an island is inside another patch, it will be identified, but if an island is inside another island that is inside a patch, it will not be identified.

\subsubsection{The GRASS mask}

GRASS has a mask command (r.mask) that can be used to limit the parts of a map that are included in an analysis. The r.le analysis programs do respond to a mask if it is present, and the results of analyses will be limited to the area specified as " 1 " in the MASK file. Moreover, when the moving window sampling method is used, the moving window will only move through the area of the map that is specified as " 1 " in the MASK file. This can considerably speed up the moving window operation, if the masked area is a small part of the map.

\subsection{The r.le.setup program}

The r.le.setup program is used to set up the sampling and analysis framework that will be used by the other r.le programs. To run r.le.setup do the following:

1. After starting grass 4.0 and setting up your location and mapset, start a GRASS monitor window using the d.mon command.

2. Move the cursor back to the command window with the GRASS command prompt $(>)$.

3. Type r.le.setup followed by a carriage return. This program runs only interactively.

4. You will now be queried for (1) the name of the map to be used as a backdrop for setting up the sampling scheme, (2) the name of a vector map to overlay on the raster map to aid in placing the sampling areas (optional), and (3) the name of a sitefile to overlay on the raster map to aid in placing the sampling areas (optional). These maps must already exist to be used here.

5. The raster map and overlay maps, if chosen, will be displayed and you will see the main r.le.setup menu.

The first menu allows the user to select how sampling will be done (regions, sampling units, moving window), setup the limits for groups and classes, and change the color table.

\subsubsection{Sampling}

Information about the structure of the landscape is obtained by overlaying a set of sampling areas on top of a map layer, and then calculating specific structural measures for the part of the map layer that corresponds to the area in each 
sampling area.

A sampling area may be one of four things (Fig. 4). First, it is possible to treat the entire map layer as the one (and only) sampling area. Second, if the map layer can be divided into meaningful geographical regions, then it is possible to treat the regions themselves as sampling areas. The third option is that the sampling areas may be sampling units of fixed shape and size (also called scale) that are placed within the map layer as a whole. The fourth and final option is that the sampling area may be moved systematically across the map as a moving window. The following sections present additional details about these options for sampling areas.

\subsubsection{Whole map layer}

If the whole map layer is to be used as the one and only sampling area (Fig. 4 ), then r.le.setup does not need to be run. The user may complete an analysis by simply entering the appropriate r.le command with the "sam $=W$ " parameter.

\subsubsection{Regions}

If regions are to be used as the sampling areas (Fig. 4), then select "REGIONS" from the first r.le.setup menu, and the user is asked to do the following:

1. "ENTER THE NEW REGION MAP NAME:". Only a new raster map name is acceptable. The user can type LIST to find out the existing raster map names in this location and mapset.

2. "PLEASE OUTLINE REGION \# 1 ". The user should move the mouse cursor into the graphic monitor window and use the mouse buttons as instructed:

Left button: where am I.

to display the current coordinates of the cursor.

Middle button: Mark a point. to enter a vertex of the region boundary.

Right button: Mark LAST point. to close the region boundary by setting the last vertex to be equal to the first one.

3. A "REGION OPTIONS:" menu is displayed and the user should use the mouse to select one of the options:

"DRAW MORE": repeat the above process and setup one more region.

"START OVER": abandon the previous setup and start all over again.

"DONE-SAVE": save the regions outlined so far and exit this procedure. 
"QUIT-NO SAVE": quit the procedure without saving the regions.

Once the "DONE-SAVE" option is selected, the new raster map of the sampling regions is generated. It is displayed on the monitor window for several seconds, the monitor window is refreshed, the main menu is displayed again, and the program is ready for other setup work.

The user can also use the GRASS r.digit or v.digit programs to digitize circular or polygonal regions and to create a sampling regions map without using r.le.setup.

\subsubsection{Sampling units}

If sampling units are to be used as the sampling areas (Fig. 4), then choose "SAMPLING UNITS" from the first r.le.setup menu. The program checks the r.le.para subdirectory for an existing "units" file from a previous setup session and allows the user to rename this file (to save it) before proceeding. The r.le.setup program will otherwise overwrite the "units" file. Then the following choice is displayed followed by a seriss of other choices:

\section{Define sampling units manually or graphically?}

The next menu allows the user to choose to define the sampling units manually or graphically. When sampling units are defined manually, the user inputs the shape and size (scale) of the sampling units by specifying dimensions in pixels using the keyboard. When sampling units are defined graphically, the user uses the mouse to define the sampling units in the GRASS monitor window, and then actually places the sampling units for each scale onto the map. By placing the units graphically the user can directly determine the method of sampling unit distribution as well as the shape, size, and number of sampling units.

If the choice is made to define sampling units manually, the user next is prompted to use the mouse to outline the actual part (or whole) of the map that will be sampled, and the following series of questions must be answered:

How many different scales do you want?

Next the user is asked to specify the number of scales that will be used. The r.le programs allow the user to simultaneously sample the same map with the same measures at several scales. Currently there can be between 1 and 15 scales that can be sampled simultaneously. Substantial output can be produced if many scales are used. 
Methods of sampling unit distribution

Sampling units must be placed spatially into the landscape. There are five options for doing this (Fig. 4):

1. Random nonoverlapping: Sampling units are placed in the landscape by randomly choosing numbers that specify the location of the upper left corner of each sampling unit, subject to the constraint that successive sampling units not overlap other sampling units or the edge of the landscape.

2. Systematic contiguous: Sampling units are placed side by side across the rows. If the placement of sampling units produces an imperfect fit (leftover area), then the left over area is shifted to the upper left corner of the sampling area, and a randomly selected starting point is chosen within the leftover area.

3. Systematic noncontiguous: The user must specify the amount of spacing (in pixels) between sampling units. Horizontal and vertical spacing are identical. Leftover area is allocated as it was with systematic contiguous sampling. Sampling units are again placed side by side (but spaced) across the rows. If the placement of sampling units produces an imperfect fit (leftover area), then the leftover area is shifted to the upper left corner of the sampling area, and a randomly selected starting point is chosen within the leftover area.

4. Stratified random: The user must specify the vertical and horizontal locations of strata, which partition the sampling area. The strata are rectangular areas within which single sampling units are randomly located.

5. Centered over sites: The user must specify the name of a sitefile containing point locations. A single sampling unit is placed with its center over each site in the site file. This is a useful approach for determining the landscape structure around points, such as around the location of wildlife observations.

The ratio (cols/rows-as a real number) for sampling unit of scale $n$ ?

The user is next prompted to enter a ratio that defines the shape of the sampling units. Sampling units may have any rectangular shape, including square as a special case of rectangular. Rectangular shapes are specified by entering the ratio of columns/rows (horizontal dimension/vertical dimension) as a real number. 
Figure 4

\section{SAMPLING AREAS}

WHOLE MAP LAYER

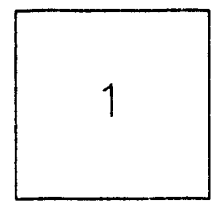

REGIONS

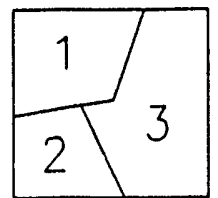

\section{SAMPLING UNITS}

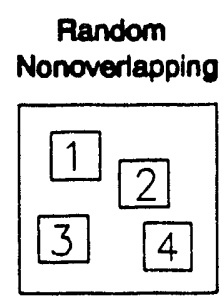

Systematic
Contiguous
\begin{tabular}{|l|l|l|}
\hline 1 & 2 & 3 \\
\hline 4 & 5 & 6 \\
\hline 7 & 8 & 9 \\
\hline
\end{tabular}

Systematic Noncontiguous

Stratified

Centered

Over Sltes

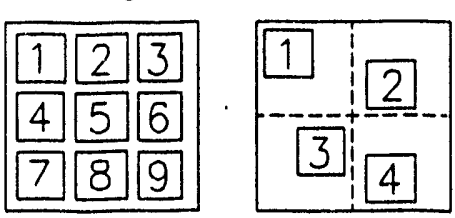

$1 \cdot \square^{2}$
$3 \cdot \bullet^{4}$

MOVING WINDOW

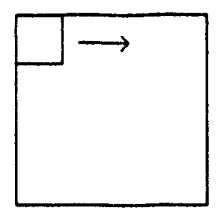


Maximum size is $m$. What size for each sampling unit of scale $n$ ?

The user is then given the maximum possible size for a sampling unit (in pixels) and asked to input the size of sampling units at each scale. Sampling units can be of any size, but the maximum size is the size of the landscape as a whole. All the sampling units, that make up a single sampling scale, are the same size.

Maximum units in scale $n$ is $p$. How many sampling units do you want?

The maximum number of units that can be placed over the map, given the shape and size of the units, is then given. The user can then choose the number of sampling units to be used in the map layer. It may not always be possible to choose the maximum number, depending upon the shape of the sampling units.

\subsubsection{Moving window:}

Using this procedure a rectangular "window" or single sampling area is moved systematically across the map to produce a new map. This sampling procedure can only be used with the measures that produce a single value or with a single class or group when measures produce distributions of values (Table 1). The first class or group specified when defining class or group limits (section 2.3.2.) is used if distributional measures are chosen with the moving window sampling method. In this case, the user should manually edit the r.le.para/recl_tb file so that the desired group is listed as the first group in this file.

Sampling begins with the upper left corner of the window placed over the upper left corner of the total area to be sampled. It is strongly recommended that the user read the section on the GRASS mask (section 2.2.2) prior to setting up the moving window, as this mask can be used to speed up the moving window operation. The value of the chosen measure is calculated for the window area. This value is assigned to the location on the new map layer corresponding to the center pixel in the window if the window has odd (e.g. $3 \times 3$ ) dimensions. The value is assigned to the location on the new map layer corresponding to the first pixel below and to the right of the center if the window has even dimensions le.g 6 $X$ 10). If this pixel has the value "O," which means "no data" in GRASS, then this pixel is skipped and a value of " $O$ " is assigned to the corresponding location in the new map. The window is then moved to the right (across the row) by one pixel, and the process is repeated. At the end of the row, the window is moved down one pixel, and then back across the row. This option produces a new map layer, whose dimensions are smaller by approximately $(\mathrm{m}-1) / 2$ rows and columns, where $m$ is the number of rows or columns in the window.

If the "MOVE-WINDOW" option in the main menu is selected, first the program checks for an existing "move_wind" file, in the r.le.para subdirectory, containing moving window specifications from a previous session. The user is given the option to avoid overwriting this file by entering a new file name for the 
old "move_wind" file. The user is then prompted to do the following:

1. "Use the mouse to define the moving window?"

If yes, then the functions of the three mouse buttons are displayed: check size, move cursor, and define the moving window. The movirig window is defined in the same way as a sampling unit. Once defined, it will be displayed wherever it is defined.

If no, then the prompt "Enter COLUMNS \& ROWS of the window:" is displayed. Type in the number of rows and columns of the moving window using the GRASS cell array coordinate system. Then the moving window will be displayed in the topleft corner of the current window of the monitor screen.

2. "Will the moving window search the whole map?"

If yes, the module saves the input and returns to the main menu.

If no, the button instruction is displayed and the user should use the mouse cursor to outline the area that the moving window will search through. This is done in the same way as above. After the area is defined and displayed wherever it is defined the user is asked "Is the search area ok?" If not, the process is repeated. Otherwise the area is displayed, with the moving window in c different color, at the top-left corner of the area. The picture is displayed for a few seconds and then it is refreshed. Then the main menu is redisplayed for next selection.

\subsubsection{Group/class limits}

Group/class limits are setup by choosing "GROUP LIMITS" from the main menu upon starting r.le.setup. The program checks for existing group/class limit files in subdirectory r.le.para and allows the user to rename these files prior to continuing. The files are named recl_tb (attribute group limits), size (size class limits), shape_PA (shape index class limits for perimeter/area index), shape_CPA (shape index class limits for corrected perimeter/area index), and shape_RCC (shape index class limits for related circumscribing circle index). If the files are not renamed the program will overwrite them.

The r.le programs allow the attribute categories in the input map to be reclassed into several attribute groups, and reports the analysis results by each of these attribute groups. The same reclassing can be done with the me. asurement indices (e.g., size), except that each "cohort" (class) of the reclassed indices is called an index class instead of a group.

Attribute groups and index classes are defined in a different way. In the r.le programs attribute groups are defined as in the following example:

$1,3,5,7,9$ thru $21=1$ (comment) 
31 thru $50=2$ (comment)

end

In this example, the existing categories $1,3,5,7,\{9,10, \ldots 20,21\}$ are included in the new group 1 , while $\{31,32,33, \ldots, 49,50\}$ are included in the new group 2. The characters in bold are the "key words" that are required in the definition. Each line is called one "reclass rule".

In the special case of the attribute groups for distance measurement using method 4, in which the "from" attribute group and "to" attribute group must be specified, the "from" and "to" groups are defined in a slightly different way, as in the following example:

\section{$1,3,5,7,9$ thru 21 end (comment)}

Here, the key word "end" is at the end of the line instead of in a new line. This rule is only used in the definition of the "from" and "to" attribute groups, because in this case both groups have one and only one reclass rule.

The GRASS reclass convention is adopted here with a little modification (see "r.reclass" command in the GRASS manual by Shapiro et al. 1989). The difference is that r.le only allows one rule for each group while the GRASS r.reclass command allows more than one. The definition of "from" and "to" groups is simply the extension of the GRASS reclass rule. The advantage of using the GRASS reclass convention is that the user can generate a permanent reclassed map, using GRASS programs, directly from the r.le setup results.

The r.le measurement index classes are defined by the lower limits of the classes, as in the following example:

$$
0.0,10.0,50.0,200.0,-999
$$

This means:

if $v>=0.0$ and $v<10.0$ then $v$ belongs to index class 1 ;

if $v>=10.0$ and $v<50.0$ then $v$ belongs to index class 2;

if $v>=50.0$ and $v<200.0$ then $v$ belongs to index class 3;

if $v>=200.0$ then $v$ belongs to index class 4;

where $v$ is the calculated index value and -999 marks the end of the index class definition. The measurement index can be the size index, one of the three shape indices, or one of the three distanc indices.

The program is currently designed to allow no more than 25 attribute groups, 25 size classes, 25 shape index classes, and 25 distance index classes.

As an alternative, the user may want to permanently group certain attributes prior tc entering the r.le programs. For example, the user may want to group attributes $1-10$, in a map whose attributes are ages, into a single attribute representing young patches. The user can do this using the GRASS r.reclass and r.resample commands, which will create a new map layer that can then be analyzed 
directly (without setting up group limits) with the r.le programs.

\subsubsection{Color table}

The user may want to change the color table for the map in the GRASS monitor window to make the sampling areas, cursor, and rubber band more visible. There are several different color tables that can be tried until a suitable one is found. Note that if you choose one of the other color tables from the menu, the color table for that GRASS raster map gets changed. To change it back to what it was originally, select "SET-OLD" from the color table menu.

If the "Color Table" option in the main menu is selected, a menu titled "SEL. NEW COLOR TYPE" is displayed that has the following options:

"ASPECT": generate a color table for aspect data.

"GREY SCALE": generate a grey scale color table. Each color is a level of grey, increasing from black to white.

"RAMPS": generate a color table with 3 sections: red only, green only, and blue only, each increasing from none to full intensity. This table is good for continuous data such as ages.

"RAINBOW": generate a Color table based on rainbow colors. the table generated here uses yellow, blue, indigo, violet, red. This table is good for continuous data such as ages.

"RANDOM": generate random colors. Good as a first pass at a color table for nominal data. This option generates different color combinations for the color table each time. Therefore it can be used repeatedly until the satisfactory colors are displayed.

"RED-YEL-GRN": generate a color table similar to that of "RAINBOW", except that the table starts at red, passes through yellow, and ends with green.

"WAVE": generate a color table with 3 sections: red only, green only, and blue only, each increasing from none to full intensity and back down to none. This table is good for continuous data like ages.

"SET OLD": assign the original color table to the input cell map if none of the above options improves the display during setup.

After one of these options is selected, the menu titled "WHAT'S NEXT" is displayed that has the following options:

"START OVER": go back to the previous menu and reset the original color table.

"MAIN MENU": exit from the color change module and go back to the main menu. The color table will be reset to that of the input file when the user leaves the r.le.setup program.

"SAVE COLOR TAB.": exit from this module and permanently save the 
currently displayed color table.

"QUIT SETUP": quit the r.le.setup program and don't save any changes.

The user can restore a user-developed color table (stored in "rules.file") by typing at the GRASS prompt "cat rules.file | r.colors map=name color=rules".

\subsection{Syntax for the r.le analysis programs}

The r.le analysis programs include r.le.dist, r.le.patch, and r.le.tex. These programs are designed to do landscape ecological analyses by computing the spatial measures selected from the measure list available with each program. Each program will be explained in the following sections. All three r.le analysis programs can be started at the GRASS prompt ( $>$ ) using either a command-line or interactive method.

The interactive version of each program is invoked by simply typing the command followed by a carriage return. The GRASS parsing routine will then ask the user to answer questions and specify parameter values. The possible parameter values are listed along with a brief summary of their meanings.

The command-line version of each program is invoked by typing the name of the program, followed by a list of parameters and parameter values, on the command line, followed by a carriage return. Each command-line parameter is described briefly in help menus for each of the programs. To invoke the commandline help menu, type the name of the program, a space, and the word "help" (e.g. r.le.tex help).

\subsection{The r.le.dist program}

The r.le.dist program can be used to measure distances between patches and report those distances using several methods. See section 2.4. for an explanation of how to start the r.le.dist program. The syntax for the command-line version and the parameters for both interactive and command-line versions are as follows:

$$
\begin{aligned}
& \text { r.le.dist } \operatorname{map}=\text { name } s a m=\text { name } \text { run }=\text { name }[\text { reg }=\text { name }] \text { ski }=\text { value } \text { can }=\text { value } \\
& \text { di1 }=\text { namel }, \text { name,...] di2 }=\text { value }[, \ldots] \text { [out }=\text { name }]
\end{aligned}
$$

where:

brackets [] indicate optional parameters or values

map is the GRASS raster map to be analyzed. This raster map must be available in the user's working GRASS database (/location/mapset/), sam is the kind of sampling area: $W, U, M$, or $R$, where $W=$ whole map, $U=$ sampling units, $M=$ moving window, or $R=$ regions, run is: $F$ or $B$, where $F=$ run in foreground, $B=$ run in background, 
reg is the name of the regions map to be used when $s a m=R$,

$s k i$ is to specify whether to skip some points when searching along the patch boundary. This is used to speed up the distance calculations.

ski $<=0$ means don't skip;

ski > 0 means:

if $n p>s k i+50$ - search every other boundary point;

if $n p>s k i+200$ - search every third boundary point;

if $n p>s k i+500$ - search every fourth boundary point;

if $n p>s k i+2000$ - search every fifth boundary point;

where $n p$ is the number of total boundary pixels of a patch. This is effective with the center-edge and edge-edge distance measures. The default value is $s k i=1$, and maximum value is 10 .

can is the maximum number of candidate patches on the nearestneighbor-list for searching the nearest neighbor patch. It means when searching the nearest patch of a particular patch, find can "close" patches first using simple method, then find out the nearest patch from these can candidate patches with point-by-point calculation. The legal range of can is 1-30. If $\operatorname{can}<1, \operatorname{can}=1$; if $\operatorname{can}>30, \operatorname{can}=30$. The default value is $\operatorname{can}=5$.

di1 is the distance measure, which can have these values:

11 = Mean distance: This is simply the total of all the distances divided by the number of distances measured.

12 = Standard deviation of distance: This is the standard deviation of the distances in the sampling area. It is calculated as:

$$
s=\sqrt{\left(\frac{\sum_{i=1}^{N}\left(x_{i}-\bar{x}\right)^{2}}{N}\right)}
$$

where $x_{i}$ is distance $i, x$ is the mean distance of all the distances, and $N$ is the number of distances.

13 = Mean distance by group: This is the mean distance within the sampling area, as in $M$, but calculated separately for the patches within each group. 
14 = Standard deviation distance by group: This is the standard deviation of distances with the sampling area, as in S, but calculated separately for the patches within each group.

$15=$ Number of distances by distance class: This is a tally of the number of distances within each of up to 25 userspecified distance classes.

16 = Number of distances by distance class by group: This is a tally of the number of distances within each of up to 25 user-specified distance classes, as in C, but calculated separately for the patches within each group.

In the case of the moving window sampling method, only one of these values may be chosen, but with the whole map, regions, or sampling unit sampling methods, the following combinations of the above are the only options:

$11-2$

13

14

15

16

di2 is the distance method. Distance can be measured and summarized in a variety of ways: (1) from each patch in the sampling area or only from patches belonging to a specific attribute group (gp), (2) to all adjacent neighboring patches or only to the single nearest neighbor patch, (3) regardless of the group of the neighbor or only to patches belonging to a specific group, (4) from center to center, from center to edge, or from edge to edge. There are nine combinations of these that represent the choices for di2 (some examples are illustrated in Fig. 5):

From each patch in the sampling area to all the adjacent neighbors of the patch
MO $=\quad$ Distance is center-center
M1 = Distance is center-eage
to the nearest patch of the same gp
M2 $=\quad$ Distance is center-center
M3 = Distance is center-edge
M4 = Distance is edge-edge
to the nearest patch of a different $g p$
M5 $=\quad$ Distance is center-center
M6 = Distance is center-edge
From each patch of a specific gp
to the nearest patch of a specific gp
M7 = Distance is center-center
M8 = Distance is center-edge
M9 = Distance is edge-edge 
Figure 5
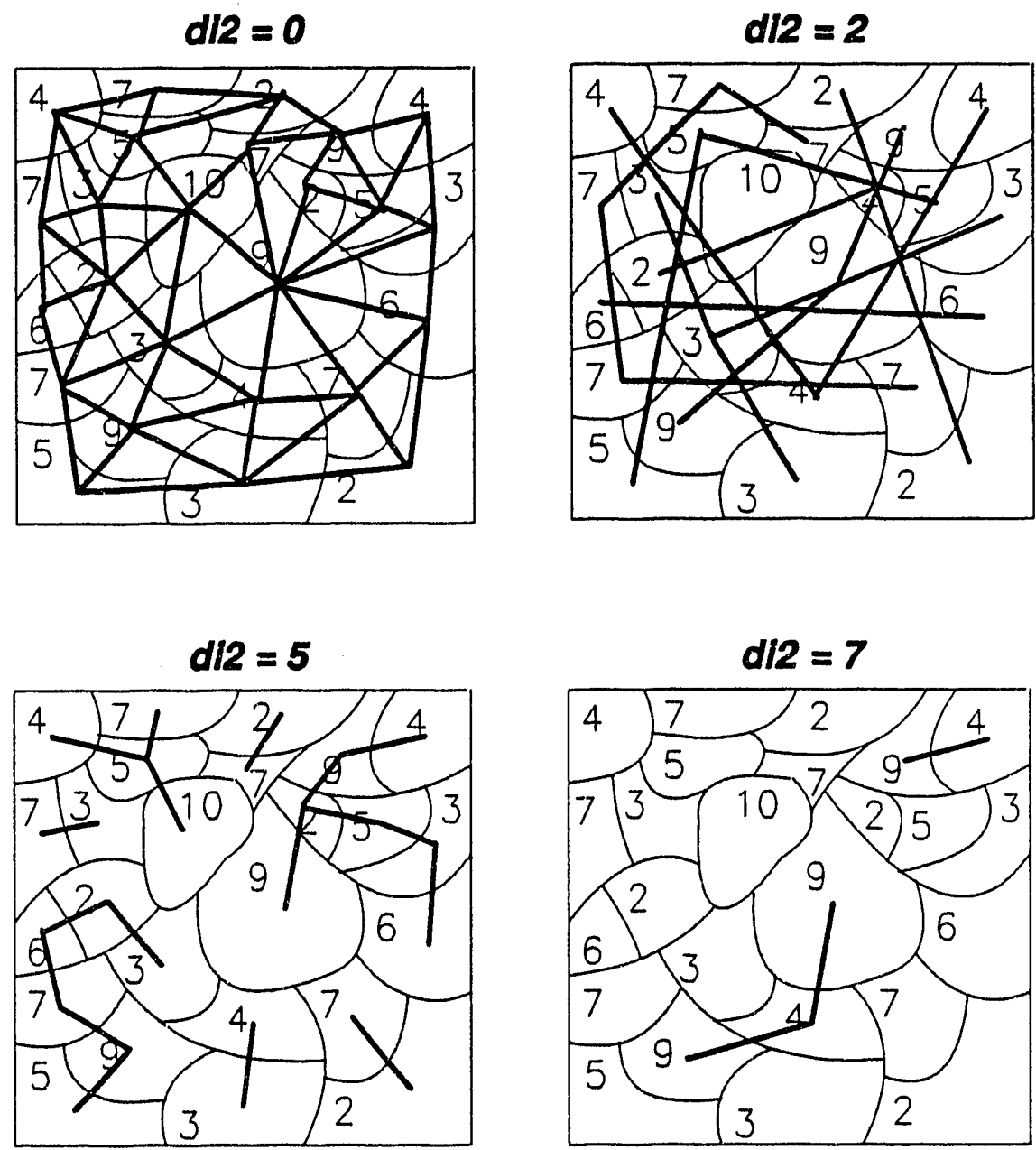
A polygon is considered to be adjacent to another polygon if it shares either an edge or a single vertex with the polygon. Polygon center $x$ and $y$ coordinates are defined as the sum of the $x$ and $y$ coordinates of all the boundary points divided by the number of points. A center of gravity algorithm for finding polygon centers will be implemented in the next version. Distance from center to edge is measured from the center of one polygon to the closest point on the boundary of the other polygon. Distance from edge to edge is measured as the minimum distance between any points on the boundary of the two polygons. Note that with methods $0-1$ a very large number of distances are calculated, whereas with methods 2-6 the number of distances measured is the same as the number of patches in the sampling area. With methods 7-9 the number of distances is the same as the number of patches in the sampling area that belong to the "from" group. Note that the distance between polygons $A$ and $B$ may be used more than once with any of the measures, as this distance may be calculated once with polygon $A$ as the "from" polygon and once with polygon $B$ as the "from" polygon. The distance between polygons $A$ and $B$ is the same no matter which is the "from" polygon if center-center distance is calculated, but it is not the same if center-edge distance is calculated.

out is the name of the output map to be used when sam $=M$

\subsection{The r.le.patch program}

This program can be used to calculate attribute, size, shape, fractal dimension, and perimeter measures for sets of patches in a landscape. See section 2.4. for an explanation of how to start the r.le.patch program. The syntax for the command-line version and the parameters for both interactive and command-line versions are as follows:

$$
\begin{aligned}
& \text { r.le.patch } \operatorname{map}=\text { name } s a m=\text { name } r u n=\text { name }[\text { reg }=\text { name }] \\
& {[a t t=\text { name }[\text {, name }, . . .]][\text { siz }=\text { name }[\text {,name,... }]][\text { sh } 1=\text { name }]} \\
& {[\text { sh2 }=\text { name }[\text {, name }, . . .]][\text { fra }=\text { name }][\text { per }=\text { name }[\text {, name }, . . .]]} \\
& \text { [out }=\text { name }]
\end{aligned}
$$

where:

brackets [] indicate optional parameters or values

map is the GRASS raster map to be analyzed. This raster map must be available in the user's working GRASS database (/location/mapset/), sam is the kind of sampling area: $W, U, M$, or $R$, where $W=$ whole map, $U=$ sampling units, $M=$ moving window, or $R=$ regions, 
run is: $F$ or $B$, where $F=$ run in foreground, $B=$ run in background, reg is the name of the regions map to be used when sam $=R$, att is a set of attribute measures:

A1 = Mean pixel attribute: This is the average value of the attributes of all the pixels in the sampling area. Each attribute is weighted by how many pixels it occupies. The mean pixel attribute, $x$, is then:

$$
\bar{x}=\frac{\sum_{i=1}^{m}\left(w_{i} * i\right)}{\text { size }}
$$

where $w_{i}$ is the number of pixels of attribute $i, i$ is the attribute of these pixels, $m$ is the number of attributes in the sampling area, and size is the size of the sampling area (in pixels). This measure is only meaningful when attributes represent interval/ratio data.

A2 = Standard deviation of pixel attribute: This is simply the standard deviation of the attributes of the pixels in the sampling area. The standard deviation of pixel attributes, $s$, is then:

$$
s=\sqrt{\left(\frac{\sum_{i=1}^{N}\left(x_{i}-\bar{x}\right)^{2}}{N}\right)}
$$

where $x_{i}$ is the attribute of patch $i, x$ is the mean attribute of all the patches, and $N$ is the number of patches.

A3 = Mean patch attribute: This is the average attribute of all the patches in the sampling area. It is calculated by summing up the attributes of each patch and dividing by the number of patches.

A4 = Standard deviation of patch attributes: This is simply the standard deviation of the attributes of the patches in the sampling area. The standard deviation of patch attributes, $s$, is then: 


$$
s=\sqrt{\left(\frac{\sum_{i=1}^{N}\left(x_{i}-\bar{x}\right)^{2}}{N}\right)}
$$

where $x_{i}$ is the attribute of patch $i, x$ is the mean attribute of all the patches, and $N$ is the number of patches.

A5 = Cover by group: This is a measure of the amount of land area covered by each group. Cover is expressed as the fraction of the sampling area occupied by each group.

A6 = Density by group: This is a measure of the number of patches in each group. It is expressed as first the fraction of the total number of patches that are in each group, and second as the raw number of patches that are in each group.

In the case of the moving window sampling method, only one of these values may be chosen, but with the whole map, region, and sampling unit sampling methods, the following combinations of the above are the only options:

A $1-4$

A5

A6

siz is a set of size measures:

S1 = Mean patch size: This measure, the mean size or area (in pixels) of the patches in the sampling area, is calculated for all patches in the sampling area, ignoring the group of each patch, by simply dividing the sampling area size by the number of patches.

S2 = Standard deviation of patch size: This is the standard deviation of the sizes (in pixels) of all the patches in the sampling area, ignoring the group of each patch. The standard deviation of patch size, $s$, is then:

$$
s=\sqrt{\left(\frac{\sum_{i=1}^{N}\left(x_{i}-\bar{x}\right)^{2}}{N}\right)}
$$

where $x_{i}$ is the size of patch $i, x$ is the mean size of all the patches, and $N$ is the number of patches. 
S3 = Mean patch size by group: This is the mean patch size within the sampling area, as in $\$ 1$, but calculated separately for all the patches within each group.

S4 = Standard deviation of patch size by group: This is the standard deviation of the sizes (in pixels) of all the patches in the sampling area, as in S4, but calculated separately for all the patches within each group.

S5 = Number by size class: This is a measure of the number of patches in the sampling area that fall within each size class. This measure is calculated for all the patches in the sampling area, ignoring the group of each patch. The results can be reported for up to 25 size classes.

S6 $=$ Number by size class by group: This is a measure of the number of patches in the sampling area that fall within each size class. This measure is calculated separately for all the patches within each group. The results can be reported for up to 25 size classes.

In the case of the moving window sampling method, only one of these values may be chosen, but with the whole map, regions, and sampling unit sampling methods, the following combinations of the above are the only options:

S1-2

S3

54

S5

S6

sh1 is a set of shape indices. There are three possible indices of patch shape here. These are only three of the simplest indices of two-dimensional shape (Austin 1984; MacEachren 1985):

M1 = Perimeter/area: The total length of the perimeter of each patch is divided by its area, and the mean of these values is then calculated. Where patches extend outside of the sampling area, the sampling area edge is considered to be the edge of the patch. A problem with the ratio of perimeter/area as a shape index is that it varies with the size of the patch.

M2 = Corrected perimeter/area: The formula for this index for each patch is: $10.282 \mathrm{X}$ perimeter $) /(\text { area })^{1 / 2}$. The mean of these values for all the patches is then calculated. This index corrects for the size problem of index $E$. The index varies from a value of 0.0 for a circle to infinity for an infinitely long and narrow shape. It is 1.12 for a square.

M3 = Related circumscribing circle: This index compares the area of the patch to the area of the smallest circle that 
shape index classes.

In the case of the moving window sampling method, only one of these values may be chosen, but with other sampling methods, the following combinations of the above are the only options:

H1-2

$\mathrm{H3}$

$\mathrm{H} 4$

$\mathrm{H} 5$

H6

fra is the fractal dimension, D, for the patches in a sampling area, and is a measure of the complexity of the perimeter. The current version implements only the perimeter-area interpretation of fractal dimension (Krummel et al. 1987). The formula for fractal dimension, $d$, is:

$$
d=2 * s
$$

where $s$ is the slope of the regression of the log of the patch perimeter versus the log of the patch area.

$\mathrm{F} 1$ = perimeter-area fractal dimension

per is the perimeter of a patch, which is the total length of external and internal boundary expressed as the number of pixel edges. The perimeter includes the edge of the sampling area when the edge of the sampling area intersects a patch. The perimeter is measured for each patch individually. Thus boundaries between adjoining patches get measured twice, once for each patch. The edge measures of r.le.tex do not measure shared boundaries twice. There are several possible measures of perimeter:

P1 = Sum of the perimeters: This is the total of all the perimeters for all the patches in the sampling area, ignoring the group to which the patch belongs.

P2 = Mean perimeter: This is the mean perimeter length for the patches in the sampling area, ignoring the group to which the patches belong. It is calculated by dividing the sum of the perimeters by the number of patches.

P3 = Standard deviation of perimeter: This is the standard deviation of perimeter length for all the patches in the sampling area, ignoring the group to which they belong. 
can circumscribe the patch. The formula for each patch is:

$$
R C C=\frac{2 *(\operatorname{area} / \mathrm{m})^{1 / 2}}{\text { longest }- \text { axis }}
$$

This index varies from 0.0 to 1.0 as the compactness of the shape approaches that of a circle. A square has the value 0.79789 .

sh2 is a set of shape measures:

$\mathrm{H} 1$ = Mean patch shape: This measure is calculated for all patches in the sampling area, ignoring the group of each patch. The mean patch shape is simply the sum of the patch shape indices for every patch divided by the number of patches.

$\mathrm{H} 2$ = Standard deviation of patch shape: This is the standard deviation of the shapes of all the patches in the sampling area, ignoring the group of each patch. The standard deviation of patch shape, $s$, is then:

$$
s=\sqrt{\left(\frac{\sum_{i=1}^{N}\left(x_{i}-\bar{x}\right)^{2}}{N}\right)}
$$

where $x_{i}$ is the shape of patch $i, x$ is the mean shape of all the patches, and $N$ is the number of patches.

$\mathrm{H} 3$ = Mean patch shape by group: This is the mean patch shape within the sampling area, as in 1, but calculated separately for all the patches within each group.

$\mathrm{H} 4$ = Standard deviation of patch shape by group: This is the standard deviation of the shapes of all the patches in the sampling area, as in 4 , but calculated separately for all the patches within each group.

$\mathrm{H} 5$ = Number by shape index class: This is the number of patches, in the sampling area, whose shape index value falls within each shape index class. This measure is calculated for all the patches in the sampling area, ignoring the group of each patch. The results can be reported for up to 25 shape index classes.

H6 = Number by shape index class by group: This is the number of patches, in the sampling area, whose shape index value falls within each shape index class. This measure is calculated separately for all the patches in each group. The results can be reported for up to 25 
The standard deviation of perimeter length, $s$, is then:

$$
s=\sqrt{\left(\frac{\sum_{i=1}^{N}\left(x_{i}-\bar{x}\right)^{2}}{N}\right)}
$$

where $x_{i}$ is the perimeter length of patch $i, x$ is the mean perimeter length of all the patches, and $N$ is the number of patches.

P4 = Sum of the perimeters by group: This is the total of all the perimeters for all the patches in the sampling area, as in P1, calculated separately for the patches belonging to each group.

P5 = Mean perimeter by group: This is the mean perimeter length for the patches in the sampling area, calculated separately for the patches belonging to each group. It is calculated by dividing the sum of the perimeters by the number of patches within each group.

P6 = Standard deviation of perimeter by group: This is the standard deviation of perimeter length for all the patches in the sampling area, as in P3, but calculated separately for the patches belonging to each group.

In the case of the moving window sampling method, only one of these values may be chosen, but with the whole map. regions, and sampling unit sampling methods, the following combinations of the above are the only options:

P1-3

P4

P5

P6

out is the name of the output map to be used when sam $=M$

\subsection{The r.le.tex program}

The r.le.tex program contains a set of measures of diversity, texture, juxtapovicion, and edge. See section 2.4. for an explanation of how to start the r.le.tex prngram. The syntax for the command-line version and the parameters for both interactive and command-line versions are as follows:

$$
\begin{aligned}
& \text { r.le.tex map = name sam = name run=name }[\text { reg }=\text { name }][\text { div = name }] \\
& {[\text { te } 1=\text { name }][\text { te } 2=\text { name }][\text { jux =name }][\text { edg=name }]} \\
& {[\text { out }=\text { name }]}
\end{aligned}
$$


where:

brackets [] indicate optional parameters or values

map is the GRASS raster map to be analyzed. This raster map must be available in the user's working GRASS database (/location/mapset/),

sam is the kind of sampling area: $W, U, M$, or $R$, where $W=$ whole map, $U=$ sampling units, $M=$ moving window, or $R=$ regions,

run is: $F$ or $B$, where $F=$ run in foreground, $B=$ run in background,

reg is the name of the regions map to be used when sam $=R$,

div is a set of measures of the diversity of patch attributes within the sampling area. The relative merits of the following measures have been evaluated by Peet (1974):

D1 = Richness: This is simply the number of different patch attributes present in the sampling area.

D2 = Shannon index $\left(H^{\prime}\right)$ : This is an index that combines richness and evenness. Its formula is:

$$
H^{\prime}=-\sum_{i=1}^{m} p_{i} * \ln \left(p_{i}\right)
$$

where $\rho_{i}$ is the fraction of the sampling area occupied by attribute $i$, and $m$ is the number of attributes in the sampling area.

D3 = Dominance: This index is related to the Shannon index, but emphasizes the deviation from evenness. The formula for dominance, $D$, is:

$$
D=\ln (n)-H^{\prime}
$$

where $n$ is the number of attributes in the sampling area. This index was first proposed and used by $O^{\prime}$ Neill et al. (1988).

D4 = Inverse Simpson's index (1/S): This index also combines richness and evenness. It is a measure of the probability of encountering two pixels of the same attribute when taking a random sample of two pixels. Its formula is:

$$
1 / S=1 / \sum_{i=1}^{m} p_{i}^{2}
$$

where $p_{i}$ is the fraction of the sampling area occupied by attribute $i$, and $m$ is the total number of attributes 
within the sampling area.

In the case of the moving window sampling method, only one of these values may be chosen, but with the whole map, regions, and sampling unit sampling methods, the following combination of the above is the only option:

D1-4

te 1 is a set of texture measures that quantify the adjacency of similar attributes. They are in a sense simply local (neighborhood) measures of diversity. Most of the measures have been reviewed by Haralick et al. (1973), Haralick (1975), and Musick and Glover (1990). All of the measures require calculation of a grey-level co-occurrence matrix (GLCM), which is $m X m$, where $m$ is the number of attributes in the sampling area. The GLCM matrix contains entries, $P_{i j}$, which are the total number of times that attribute $i$ is adjacent to attribute $j$. The total number of adjacencies is calculated by moving through the sampling area pixel-by-pixel. There are five measures of texture that can be calculated:

M1 = Contagion: This measure quantifies the degree of clumping, and is a modification of the entropy measure (N). The formula for contagion, $C$, is:

$$
C=2 * \ln (m)-E N T
$$

The formula for this measure was printed incorrectly in O'Neill et al. (1988) as $2 m^{*} \ln (m)-E N T$.

M2 = Angular second moment: This is a measure of the homogeneity of the landscape. Larger values indicate more homogeneity. The formula for angular second moment, $A S M$, is:

$$
A S M=\sum_{i=1}^{m} \sum_{j=1}^{m}\left(P_{i j}\right)^{2}
$$

M3 = Inverse difference mornent: The formula for inverse 
difference moment, IDM, is:

$$
I D M=\sum_{i=1}^{m} \sum_{j=1}^{m} \frac{1}{1+(i-j)^{2}} P_{i j}
$$

M4 = Entropy: Entropy is a maximum when all pixels of attribute $i$ are as far apart from one another as possible. The formula for entropy, ENT, is:

$$
E N T=-\sum_{i=1}^{m} \sum_{j=1}^{m} P_{i j} * \ln \left(P_{i j}\right)
$$

M5 = Contrast: This is a measure of the contrast or amount of local variation present in the landscape. The formula for contrast, CON, is:

$$
\operatorname{CON}=\sum_{i=1}^{m} \sum_{j=1}^{m}\left[(i-j)^{2} * P_{i j}\right]
$$

In the case of the moving window sampling method, only one of these values may be chosen, but with the whole map, region, and sampling unit sampling methods, the following is the only option, and it must be chosen, along with te2, to get texture measurements:

M1-5

te 2 is a set of seven methods for analyzing the adjacencies for each

$$
\begin{aligned}
& \text { pixel (Fig. 6): } \\
& \text { T1 }=2 \mathrm{~N}-\mathrm{H} \\
& \mathrm{T} 2=2 \mathrm{~N}-45 \\
& \mathrm{~T} 3=2 \mathrm{~N}-\mathrm{V} \\
& \mathrm{T} 4=2 \mathrm{~N}-135 \\
& \mathrm{~T} 5=4 \mathrm{~N}-\mathrm{HV} \\
& \mathrm{T} 6=4 \mathrm{~N}-\mathrm{DIAG} \\
& \mathrm{T} 7=8 \mathrm{~N}
\end{aligned}
$$

jux is a set of two juxtaposition measures. Juxtaposition was described and used by Mead et al. (1981) and Henein and Cross (1983). Juxtaposition is a measure of the weighted length of edges surrounding a center pixel. 
figure 6

\section{TEXTURE METHODS}
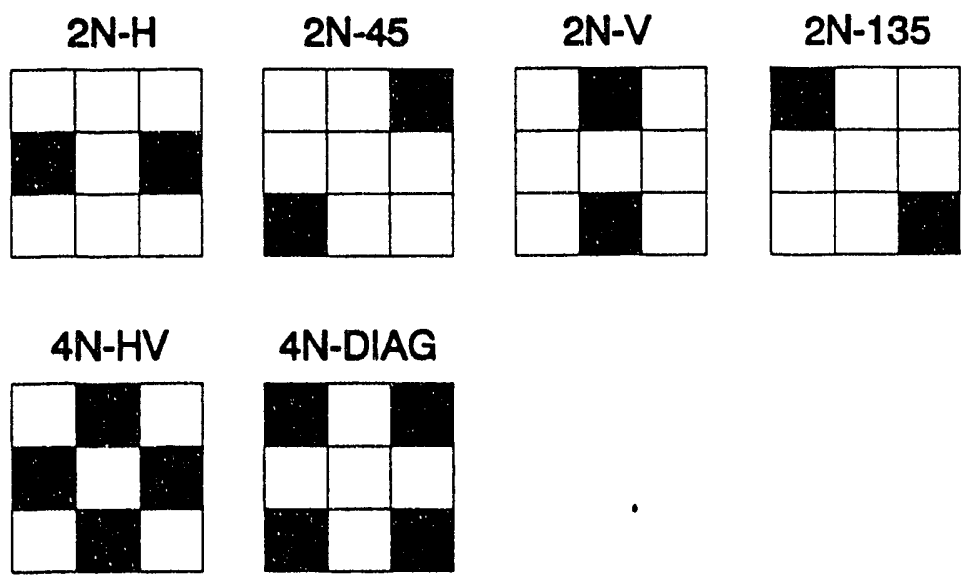

$8 N$

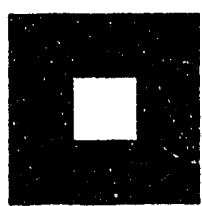


The juxtaposition for a center cell surrounded by eight neighbors is given by:

$$
J=\frac{\sum_{n=1}^{8} q_{n} * w_{i j}}{\sum_{n=1}^{8} q_{n}}
$$

where $q_{n}$ is 2.0 if cell $n$ horizontally or vertically shares an edge and is 1.0 if cell $n$ diagonally shares and edge, and $w_{i 1}$ is a number between -1.0 and +1.0 which indicates the relative "quality" or weight to be given to edges between attributes $m_{1}$ and $m_{1}$.

In calculating juxtapcsition, diagonal neighbors get a quantity ranking, $q$, of 1.0 , while horizontal and vertical neighbors get a quantity ranking of 2.0 because horizontal and vertical edges share more edge than do diagonal edges in a raster representation of patches.

Weights, $w_{i j}$, are assigned by the user to edges between different attributes. A weighting matrix, which is $m$ $X m$, where $m$ is the number of different attributes, must be created with an editor and stored as an ASCII file named "weight" in the r.le.para subdirectory. The weighting matrix has the format:

$$
\begin{array}{ccccc} 
& a t t_{1} & a t t_{2} & \ldots & a t t_{m} \\
a t t_{1} & w_{11} & w_{12} & \ldots & w_{1 m} \\
a t t_{2} & w_{21} & w_{22} & \ldots & w_{2 m} \\
\ldots & \ldots & \ldots & \ldots & \ldots \\
a t t_{m} & w_{m 1} & w_{m 2} & \ldots & w_{m m}
\end{array}
$$

where $a t t_{i}$ is attribute $i$, expressed as an integer, of $m$ attributes, and $w_{i j}$ is the weight, expressed as a real number between -1.0 and +1.0 , assigned when attribute $i$ and attribute $j$ share an edge. The two juxtaposition measures, then, are:

$\mathrm{J1}=$ Mean juxtaposition: The program first calculates the juxtaposition for each pixel in the map layer by examining edges with attributes in the 8 cells surrounding each pixel. Then the program finds the 
mean juxtaposition of all the cells in the sampling area, by summing all the juxtaposition values and dividing by the number of pixels.

$\mathrm{J} 2$ = Standard deviation of juxtaposition: This is the standard deviation for all the pixels in the sampling area. The standard deviation, $s$, is given by:

$$
s=\sqrt{\left(\frac{\sum_{i=1}^{N}\left(x_{i}-\bar{x}\right)^{2}}{N}\right)}
$$

where $x_{i}$ is the juxtaposition for cell $i, x$ is the mean juxtaposition of all the cells, and $N$ is the number of cells.

In the case of the moving window sampling method, only one of these values may be chosen, but with the whole map, region, and sampling unit sampling methods, the following combination of the above is the only option:

$\mathrm{J} 1-2$

edg is the length of patch boundary, but in summing edge for all the patches in a sampling area edges are counted only once when they are shared between two patches. An "edge" is considered to occur only between adjoining cells along a row or within a column. Diagonal neighbors with different attributes are not considered edge.

$\mathrm{E} 1$ = Sum of the edges: This is the total length of all the edges, counted only once, of all the patches in the sampling area. It differs from the total perimeter length which sums the length of each patch's total perimeter, effectively counting shared perimeters twice.

out is the name of the output map to be used when sam $=M$

\subsection{Output from the r.le analysis programs}

\subsubsection{Moving window output maps and their interpretation}

All three of the r.le analysis programs produce a new raster map when the moving window method of sampling is used. The name of the output map is specified with the "out" parameter. These maps are stored in the conventional GRASS database under the location and mapset in use at the time of the analysis.

GRASS allows raster maps to contain only integers, and this presents problems for analyses that produce real numbers as output (e.g., r.le.tex's Shannon 
diversity measure). For this reason, the results of analyses for most measures are multiplied by $1,10,100$, or 1000 to produce multiplied integer representations of the actual real numbers that should be output. Table 2 contains a list of each of the measures and the multiplier used. At the present time, these multipliers are embedded in the source code. In the future, they will be stored in a file, that can be edited, in the r.le.para subdirectory.

All moving window output maps are assigned a green-yellow-red color table, which can be changed by the user with the GRASS r.colors command.

2.8.2. Output files and data formats from non-moving window analyses

Several output files are produced by the r.le programs. Output files are stored in the r.le.out subdirectory. The output files and their format are as follows $(g p=$ attribute group):

From r.le.dist:

11-2.OUT

Scale Sampl. area\# Mean distance Standard deviation distance

13.OUT

Scale Sampl. area\# Mean distance by gp

14. OUT

Scale Sampl. area\# Standard deviation distance by gp

15.OUT

Scale Sampl. area\# Number of distances in ea. distance class

16.OUT

Scale Sampl. area\#

No. in ea. index class-gp 1

No. in ea. index class-gp 2

... $\ldots$

No. in ea. index class-gp n 
TABLE 2. Measures whose output value is a multiple of the real value, in maps output using the moving window sampling method. This multiplication is necessary because GRASS does not allow raster maps to have real numbers as attributes. Small real numbers would thus get rounded to the nearest integer (e.g., $3.24=3$ ), destroying the precision in the number.

r.le.dist

Mean distance

Multiplier

Standard deviation distance

Mean distance by gpl

standard deviation distance by gpl

100

Number of distances by distance class 1

Number of distances by distance class by gpl

100

1

1

r. le.patch

əむた

Mean pixel attribute

10

Standard deviation pixel attribute 10

Mean patch attribute

10

Standard deviation patch attribute

10

Cover by gpl

Density by gp1

1000

siz

Mean patch size

Standard deviation size

10

Mean patch size by gpl

10

Standard deviation size by gpl 10

Number by size class 1

$\operatorname{sh} 2$

Number by size class by $\mathrm{gpl}$

Mean patch shape

standard deviation shape

1000

Mean patch shape by gpl

1000

standard deviation shape by gpl

1000

Number by shape class 1

Number by shape class by gpl

Perimeter-area fractal dimension

per

Sum of perimeters

Mean perimeter

Standard deviation perimeter 10

Sum of perimeters by gpl 1

Mean perimeter by $9 p 1$

Standard deviation perimeter by gpl 10

r. le.tex

div

Richness

Shannon

Dominance

Inverse Simpson's

tel

Contagion (C)

Angular second moment (ASM)

Inverse difference moment (IDM)

1000

Entropy (ENT)

Contrast (CON) 
TABLE 2. Continued.

jux

Mean juxtaposition

1000

Standard deviation juxtaposition

1000

edg Sum of edges 
From r.le.patch:

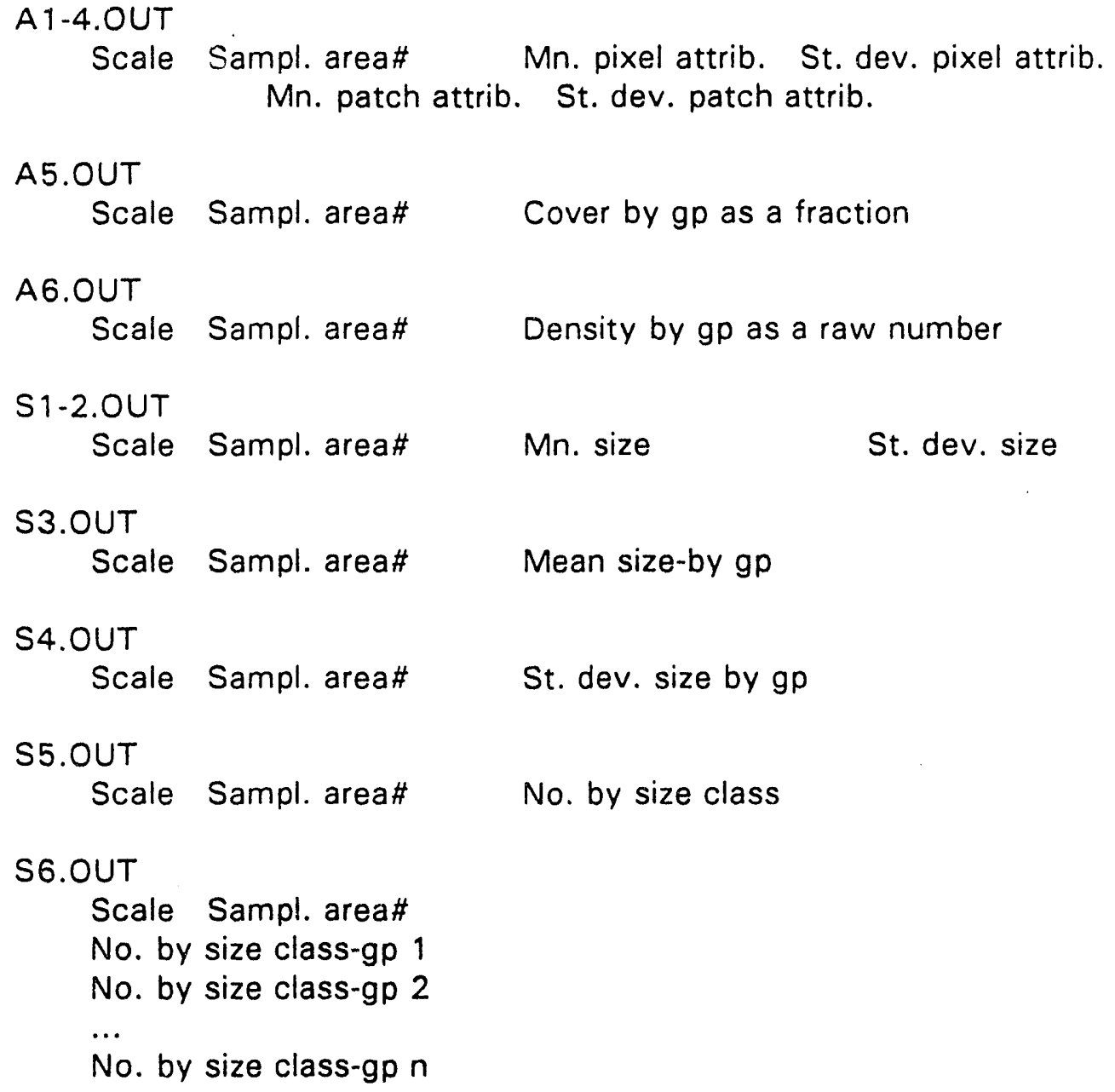




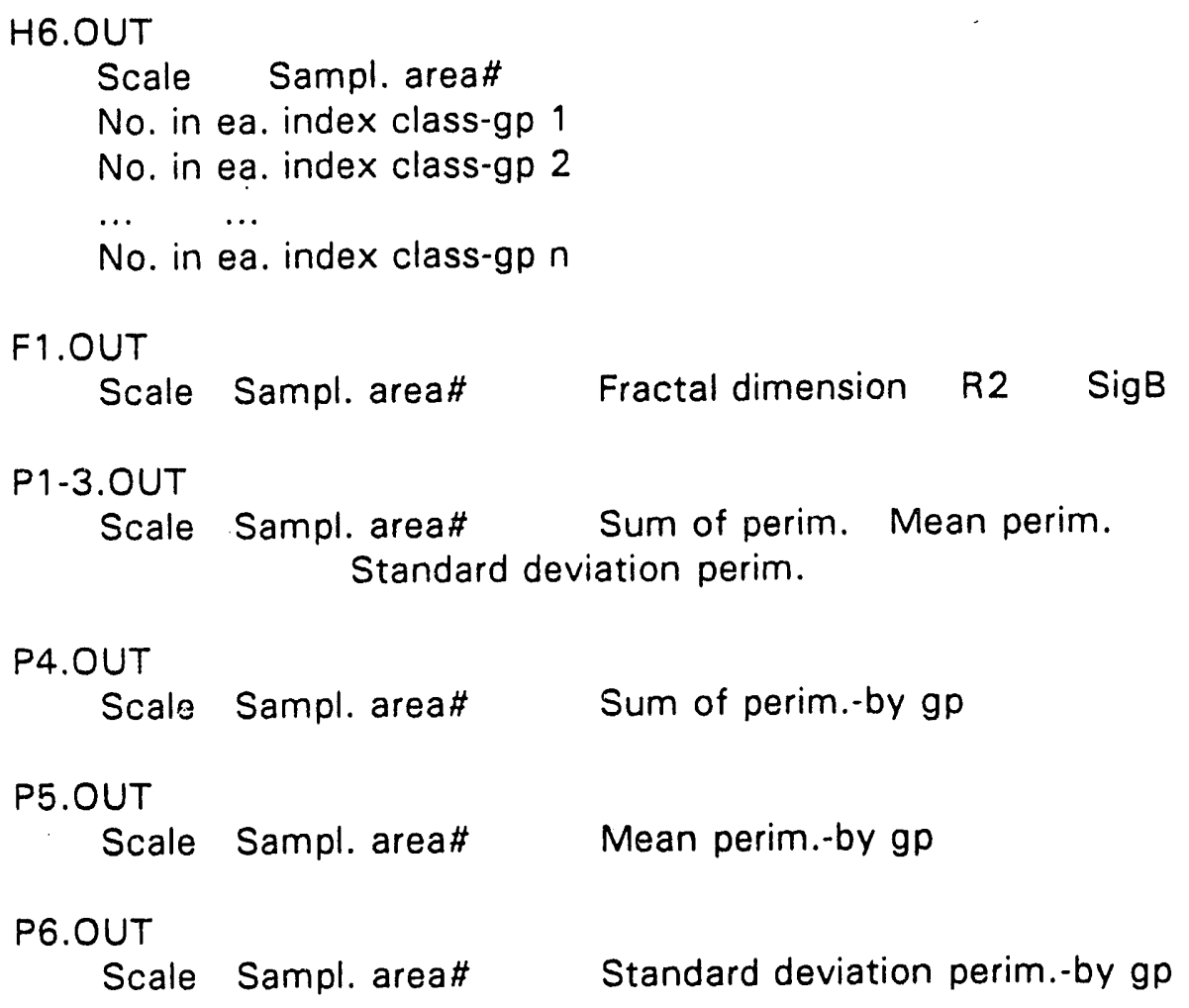

\subsubsection{Data analysis with external statistical and graphics software}

There are a number of more specialized measures of landscape structure which are not included in this program. Spatial autocorrelation analysis iGriffith 1987; Odland 1988), spatial spectral analysis (Ford and Renshaw 1984; Renshaw and Ford 1984), and geostatistics (Woodcock 1988; Mulla 1988; Oliver et al. 
$1989 a$, b) are all methods of spatial analysis that may provide useful quantitative information about landscape structure, but that also require specialized programming. Software for spatial autocorrelation analysis (Anselin 1989; Griffith 1989), spatial spectral analysis (Renshaw and Ford 1984), and geostatistical analysis (Robertson 1987) must be used separately from the r.le programs.

The data that are output by the r.le programs can be compared and analyzed graphically. There is currently no graphical display program built into the r.le programs, although maps and results from moving window analyses can be displayed using GRASS programs. The r.le program output data must be examined graphically using external software.

\subsection{The r.le.null program}

The r.le.null program is designed to generate a neutral structure map layer based on the theory of Gardner et al. (1987). This map layer can be used as a "null hypothesis" layer for testing the statistical significance of landscape measures. The syntax for the command-line version and the parameters for both interactive and command-line versions are as follows:

$$
\begin{aligned}
& \text { r.le.null } \text { map }=\text { name num }=\text { value } a t t=\text { value }[\text {, value }, . . .] \\
& \text { pro }=\text { value }[\text {, value }, . . .]
\end{aligned}
$$

where:

brackets [] indicate optional parameters or values

map is the GRASS raster map, with neutral structure, 1:0 be created, num is the number of attributes ( $\max =24$ ) to be generated within the neutral structure map. att is attribute $i$ of num attributes, where $i$ is an integer, pro is the probability for attribute $i$ of num attributes. This probability is a decimal fraction usually $<=1.0$, which indicates what fraction of the neutral structure map will contain pixels with attribute $i$. The total probability of all the attributes can be less than or greater than 1.0. If less than 1.0, the rest of the map is filled with zeroes. If greater than 1.0, then the attributes after $1.0 \%$ is reached are ignored.

\subsection{The r.le.rename program}

The r.le.rename program is used to either add a suffix to all the files in the r.le.out subdirectory, or to rename the files one by one. It is necessary to rename the files before running an r.le program over again, because the r.le program will overwrite existing files each time the program is started. The syntax for the command-line version and the parameters for both interactive and command-line 
versions are as follows:

$$
\begin{gathered}
r . l e . r e n a m e[-a][\text { ext }=\text { name }][\text { old }=\text { name }[\text {, name }, . . .] \\
{[\text { new }=\text { name }[\text {, name }, . . .]}
\end{gathered}
$$

where:

brackets [] indicate optional parameters or values

$-a$ is a flag indicating that all files in the r.le.out subdirectory that have extension ".out" will have their extension changed to that specified by parameter ext. The other files in r.le.out will not be affected.

ext specifies the new extension with which to replace the out extension of files in the r.le.out subdirectory, old is an old name for file $i$, in the r.le.out subdirectory, to be changed,

new is the new name for file $i$, in the r.le.out subdirectory

\subsection{The r.le.show program}

The r.le.show program is designed to allow the user to visually check the sampling units or moving window that was setup using r.le.setup. The syntax for the command-line version and the parameters for both interactive and commandline versions are as follows:

r.le. show $\operatorname{map}=$ name $s a m=$ name

where:

brackets [] indicate optional parameters or values

map is the raster map to display as background to show the sampling units or moving window that were setup sam specifies the type of sampling area to be displayed:

$U=$ sampling units

$M=$ moving window

After starting r.le.show the user is asked a series of questions about how and where to display the sampling units or moving window.

The first question is: "Draw a new frame in which to display sampling areas? $(y / n)[y] "$. If the user wants the sampling areas displayed in their original shape, size, and location as an overlay on the original map layer as is normally the case, then the user should answer " $n$ " to this question. Then use the mouse to select the current window, which will usually be the whole window. After selecting the window, the user will then be asked to "Please enter the sampling unit file name: 
$>>>$ " This is usually "units". The sampling units of each scale can be overlain on each other or the screen can be refreshed before displaying each new scale. The user is asked "Refresh the screen? $(y / n)[y] "$. If a moving window is to be displayed, then the moving window file name is usually "move_wind".

If the user answers " $y$ " to the first question, then the mouse can be used to outline a new window. After outlining the window, the user will then be asked to "Please enter the sampling unit file name: $>>>$ " This is usually "units". The sampling units of each scale can be overlain on each other or the screen can be refreshed before displaying each new scale. The user is asked "Refresh the screen? $(y, n)[y] "$. If a moving window is to be displayed, then the moving window file name is usually "move_wind".

\subsection{The r.le.trace program}

When sampling the whole map (sam $=W$ ), the r.le.trace program can be used to do three things: (1) display the boundary of each patch and show how the boundary is traced, (2) display the attribute, size, perimeter, and shape indices for each patch, and (3) save these data in an output file. The syntax for the commandline version and the parameters for both interactive and command-line versions are as follows:

$$
\text { r.le.trace } \operatorname{map}=\text { name }[\text { out }=\text { name }]
$$

where:

brackets [] indicate optional parameters or values

map is the name of the raster map whose patches are to be traced out is the name of an output file that can be used to store patch data

After r.le.trace is invoked, the program begins tracing and the message "r.le.trace" is working ..." is displayed. Then the user is asked "Display the traced boundary of a particular patch? $(y / n)[n] "$ If the user types " $y$ " then the program asks the user for "The patch number" that is to be displayed. The patch number is generated by the r.le.trace program as it goes through the map and traces the patches. It will not usually be known ahead of time, so this option will often not be used first. Upon entering the patch number the program displays the tracing operation in the window and then prints the patch attribute, patch size, patch perimeter, and the three patch shape indices. The program then asks "Display another patch $(y / n)[n] "$

If the user answers " $n$ " to the question "Display the traced boundary of a particular patch? $(y / n)[n] "$ then the program asks "Display the patches sequentially, with their size, perim., shape? $(y / n)[n] "$ If the user answers " $n$ " then the program quits. If the user answers " $y$ " then the user is given the following options:

$$
\begin{gathered}
<C R>\text { - show patch } \\
s \text { - skip one patch }
\end{gathered}
$$




$$
\begin{aligned}
& r \text { - refresh screen } \\
& q \text { - quit display and save }
\end{aligned}
$$

$<C R>$ begins displaying the patch boundary in white and the patch data starting with patch \#1. "s" skips the display of the next patch boundary, but prints the patch data. " $r$ " erases the boundaries from the screen and redisplays the map. " $q$ " allows the user to quit the r.le.trace program, but not erase the map layer. If the out parameter was specified, then the attribute, size, perimeter, and shape indices for each patch are saved in this file automatically. 


\section{GLOSSARY}

Attribute: One of several values that are possible for the cells in a GRASS cell file, or a polygon in a GRASS vector file.

Class: see "Index class"

Element type: a part (element) of the landscape that has been classified as belonging to a group of similar elements. For example, barns and houses are elements that might be classified as members of the "buildings" element type.

GRASS raster map: a two-dimensional raster array of integer values representing a geographic area. A GRASS raster map actually consists of the cell file itself plus several supporting files. Of these, the most important is the cell header file which contains information on the number of rows and columns in the cell file and the geographic area it covers. Other supporting files include the cell category file, the cell color table file, the cell history file, and the cell range file. These files are normally handled automatically in GRASS wherhever a raster map is manipulated. (Refer to the GRASS User's Guide and the GRASS Programmer's Manual for further information.)

GRASS vector map: a polygonal representation of a geographic area, where the polygons are formed by points and line segments (also known as nodes and arcs). GRASS vector maps consist of an arc-node file (a "dig" file) and several supporting files. The arc-node file contains a header section, which includes the geographic location of the layer, and a list of arcs and their defining points and nodes. The vector index and pointer file ("dig_plus" file) contains the polygon topology, i.e., information on which arcs and nodes comprise which polygons. The vector category attribute file ("dig_att" file) lists the category, or attribute, for each polygon, while the vector category label file ("dig_cat". file) lists labels for the categories, if they have been supplied by the user. Although in most cases, the supporting files are handled automatically by GRASS, the user must in some cases deal with them separately. For example, when performing a raster to vector conversion, it is necessary to first create the arc-node file and then the topology file. (Refer to the GRASS User's Guide and the GRASS Programmer's Manual for further information.)

Group: a reclassing of related attributes into a single unit or "group." For example all patches that have not been disturbed in $1,5,6$, or 9 years might be grouped into a 0-10 year age group.

Index class: the values of many of the indices may be reported in index classes in order to display the distribution of index values. 
Moving window: a square sampling area, with odd dimensions (e.g. 3 pixels wide by 3 pixels high), that is moved pixel-by-pixel across the rows in a map layer. At each location the center pixel is assigned the value for a particular structural measure calculated for the part of the map layer corresponding to the window area.

Sampling area: a polygon, with a certain size and shape, that identifies the area on a map layer that is to be used in calculating the r.le measures. 


\section{BIBLIOGRAPHY}

Anselin, L. 1989. Spatial regression analysis on the PC: spatial econometrics using GAUSS. Draft manual, Department of Geography and Department of Economics, University of California, Santa Barbara, Calif. 97 pp.

Austin, R.F. 1984. Measuring and comparing two-dimensional shapes. $p$. 293-312 in Gaile, G.L. and C.J. Willmott (eds.) Spatial Statistics and Models. D. Reidel Publ. Co., Boston.

Ford, E.D. and E. Renshaw. 1984. The interpretation of process from pattern using two-dimensional spectral analysis: modelling single species patterns in vegetation. Vegetatio 56:113-123.

Forman, R.T.T. and M. Godron. 1986. Landscape Ecology. John Wiley and Sons, New York. 619 pp.

Gardner, R.H., B.T. Milne, M.Cj. Turner, and R.V. O'Neill. 1987. Neutral models for the analysis; of broad-scale landscape pattern. Landscape Ecology 1:19-28.

Griffiths, D.A. 1987. Spatial Autocorrelation: A Primer. Resource Publications in Geography, Association of American Geographers, Washington, D.C. 86 pp.

Griffiths, D.A. 1989. Spatial regression analysis on the PC. Institute of Mathematical Geography, Syracuse University, Discussion Paper No. 1. $84 \mathrm{pp}$.

Griffiths, G.H. and M.G. Wooding. 1988. Pattern analysis and the ecological interpretation of satellite imagery. Proceedings of the IGARSS ' 88 Symposium [Edinburgh, Scotland-Sept. 13-16, 1988], p. 917-922.

Haralick, R.M. 1975. Statistical and structural approaches to texture. Proceedings of the IEEE 67:786-804.

Haralick, R.M., K. Shanmugam, and I. Dinstein. 1973. Textural features for image classification. IEEE Transactions on Systems, Man, and Cybernetics SMC3:610-621.

Heinen, J. and G.H. Cross. 1983. An approach to measure interspersion, juxtaposition, and spatial diversity from cover-type maps. Wildlife Society Bulletin 11:232-237.

Kennedy, S.K. and W.-H. Lin. 1986. FRACT-a FORTRAN subroutine to calculate the variables necessary to determine the fractal dimension of closed forms. 
Computers \& Geosciences 12:705-712.

Krummel, J.R., R.H. Gardner, G. Sugihara, R.V. O'Neill, and P.R. Coleman. 1987. Landscape patterns in a disturbed environment. Oikos 48:321-324.

MacEachren, A.M. 1985. Compactness of geographic shape: Comparison and evaluation of measures. Geografiska Annaler 67B:53-67.

Mead, R.A., T.L. Sharik, S.P. Prisley, and J.T. Heinen. 1981. A computerized spatial analysis system for assessing wildlife habitat from vegetation maps. Canadian Journal of Remote Sensing 7:34-40.

Milne, B.T. 1988. Measuring the fractal geometry of landscapes. Applied Mathematics and Computation 27:67-79.

Mulla, D.J. 1988. Using geostatistics and spectral analysis to study spatial patterns in the topography of southeastern Washıngton state, U.S.A. Earth Surface Processes and Landforms 13:389-405.

Musick, H.B. and H.D. Grover. 1990. Image textural measures as indices of landscape pattern. p. 77-103 in Turner, M.G. and R.H. Gardner (eds.) Quantitative methods in landscape ecology. Springer-Verlag, New York.

Odlund, J. 1988. Spatial Autocorrelation. Sage Publications, Beverly Hills, California. $87 \mathrm{pp}$.

Oliver, M., R. Webster, and J. Gerrard. 1989a. Geostatistics in physical geography. Part 1: theory. Transactions of the Institute of British Geographers 14:259-269.

Oliver, M., R. Webster, and J. Gerrard. 1989b. Geostatistics in physical geography. Part 2: applications. Transactions of the Institute of British Geographers 14:270-286.

O'Neill, R.V., J.R. Krummel, R.H. Gardner, G. Sugihara, B. Jackson, D.L. Angeles, B.T. Milne, M.G. Turner, B. Zygmunt, S.W. Christensen, V.H. Dale, and R.L. Graham. 1988. Indices of landscape pattern. Landscape Ecology 1:153162.

Peet, R.K. 1974. The measurement of species diversity. Annual Review of Ecology and Systematics 5:285-307.

Renshaw, E. and E.D. Ford. 1984. The description of spatial pattern using two-dimensional spectral analysis. Vegetatio 56:75-85.

Risser, P.G., J.R. Karr, and R.T.T. Forman. 1984. Landscape ecology: 
directions and approaches. Illinois Natural History Survey Special Publication No. 2, Champaign, Illinois. $18 \mathrm{pp}$.

Robertson, G.P. 1987. Geostatistics in ecology: interpolating with known variance. Ecology 68:744-748.

Shapiro, M., J. Westervelt, D. Gerdes, M. Higgins, and M. Larson. 1989. GRASS 3.0 Programmer's Manual. USA-CERL ADP Report N-89/14. US Army Corps of Engineers Construction Engineering Research Laboratory, Champaign, Illinois.

Urban, D.L., R.V. O'Neill, and H.H. Shugart, Jr. 1987. Landscape ecology: a hierarchical perspective can help scientists understand spatial patterns. BioScience 37:119-127.

USA-CERL. 1991. GRASS 4.0 Reference Manual. United States Army Corps of Engineers Construction Engineering Research Laboratory, Champaign, Illinois.

Woodcock, C.E., A.H. Strahler, and D.L.B. Jupp. 1988. The use of variograms in remote sensing: II. Real digital images. Remote Sensing of Environment 25:349-379. 


\section{APPENDICES}




\section{APPENDIX 1 - IIMITS}

A number of limits are embedded in the code. Exceeding these limits may produce unexpected outcomes.

Number of attribute groups

$=25$

Number of index classes

$=25$

Number of pixels in any one patch's edge

$=5000$

Number of scales that can be used simultaneously

$=15$

Number of sites when sampling units are centered over sites $=200$ 


\section{APPENDIX 2 - TIME NEEDED TO COMPLETE ANALYSES WITH THE R.LE PROGRAMS}

The r.le programs may require minutes or hours to complete a particular analysis. The amount of time required varies only with the size of the map in the case of r.le.tex. In the case of r.le.dist and r.le.patch the amount of time varies with the size of the map and its complexity (number of patches, number of islands). With the r.le.dist and r.le.patch programs much of the execution time is spent tracing the patches rather than completing measurement calculations. Thus increasing the number of measures that you select will not dramatically increase the required execution time. On a Sun Sparcstation 1 running at 12.5 MIPS it takes approximately 2 minutes to use r.le.patch with all the measurement options to analyze a $250 \times 250$ pixel map ( $\mathrm{sam}=W$ ), and about 8 minutes with a $500 \times 500$ pixel map. The r.le.dist program is a little faster. The r.le.tex program may only require 30 seconds and 2 minutes for these map sizes.

Moving window analyses can be extremely time consuming, particularly with r.le.dist and r.le.patch, where the patches must be traced inside each window. For these two programs the time required approximately doubles as the length of a side of the window doubles. Thus a $10 \times 10$ window requires about twice as much time as a $5 \times 5$ window. On a Sun Sparcstation 1 running at 12.5 MIPS r.le.tex required 15 minutes to complete a $5 \times 5$ moving window analysis of a $250 \times 250$ pixel map, while r.le.patch required approximately 5 hours for the same window and map. The amount of time required for a moving window analysis can be determined by starting the analysis and observing the expected completion time, which will be printed on screen while the program runs. The expected completion time is updated as the window moves, so allow it to run for awhile to get the best estimate. 
APPENDIX 3 - HELP MENUS FOR THE r.le ANALYSIS PROGRAMS 


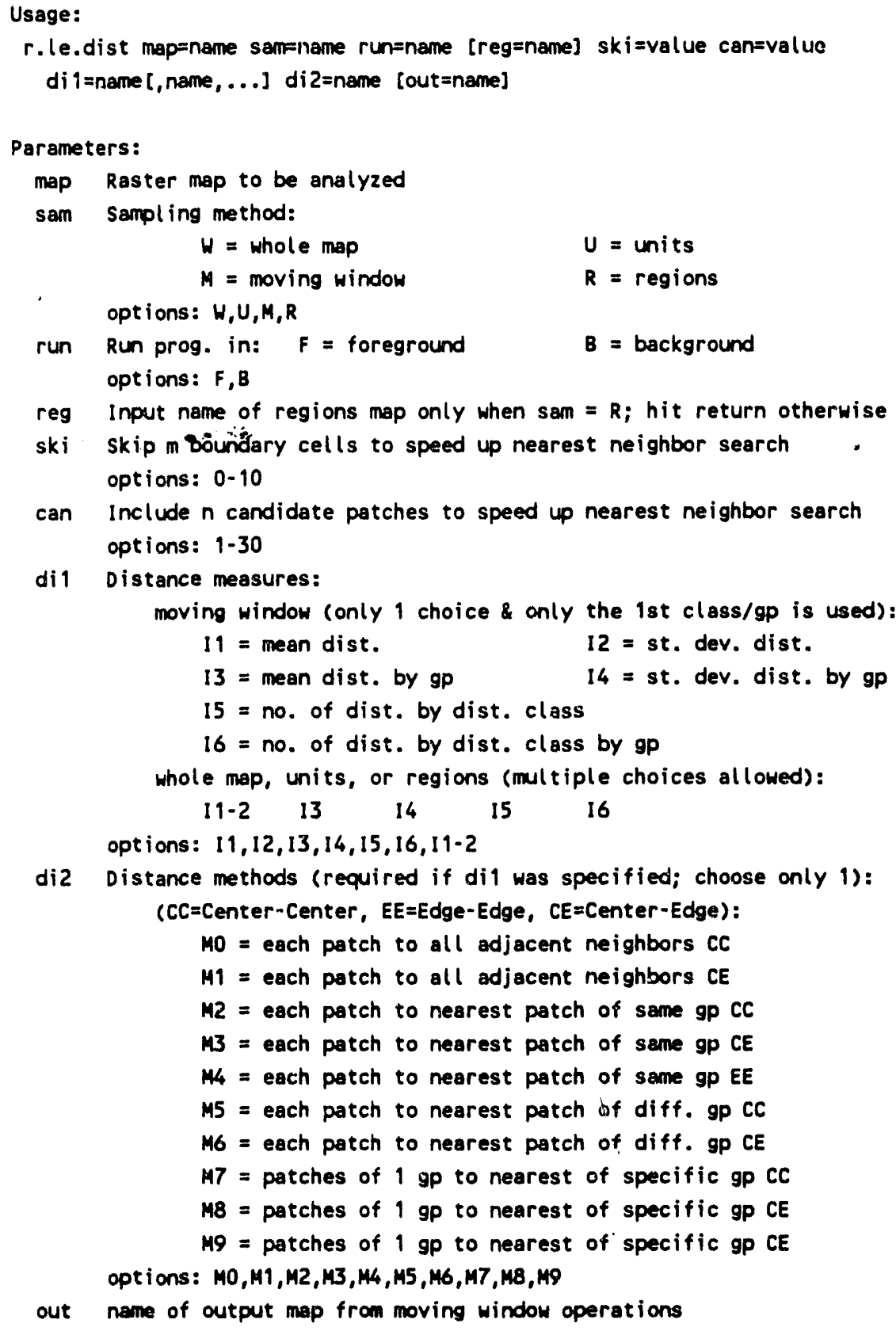




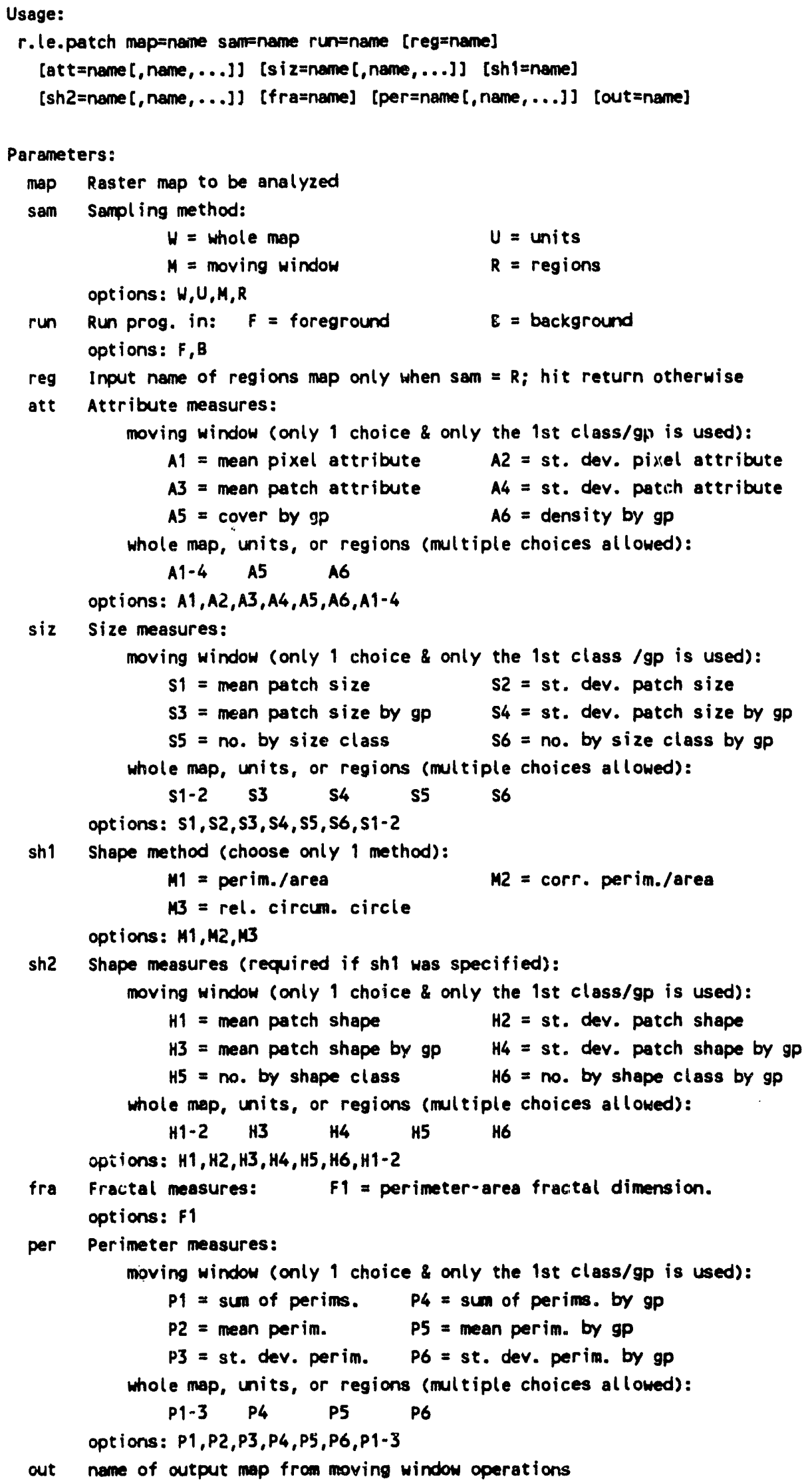

out name of output map from moving window operations 


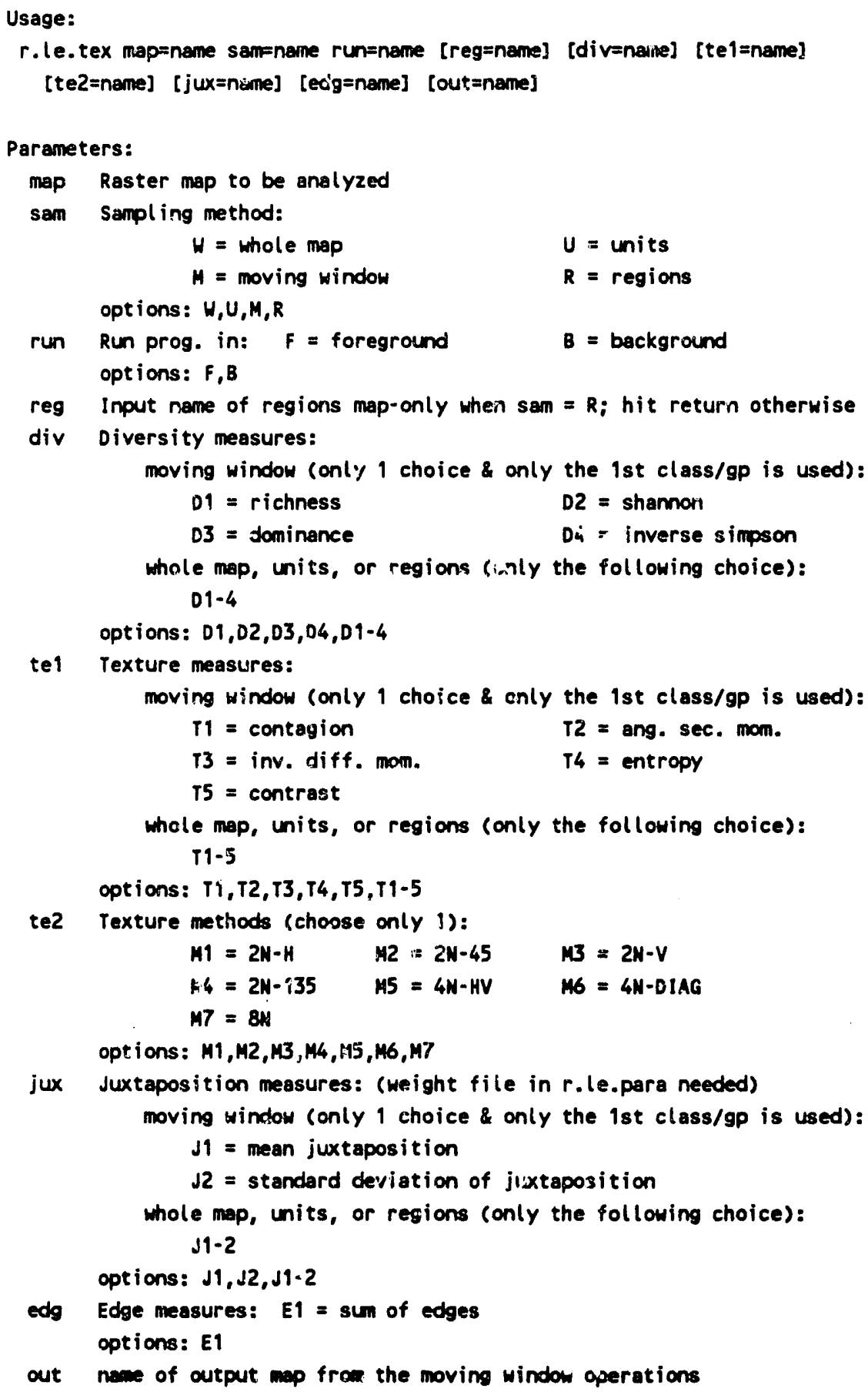



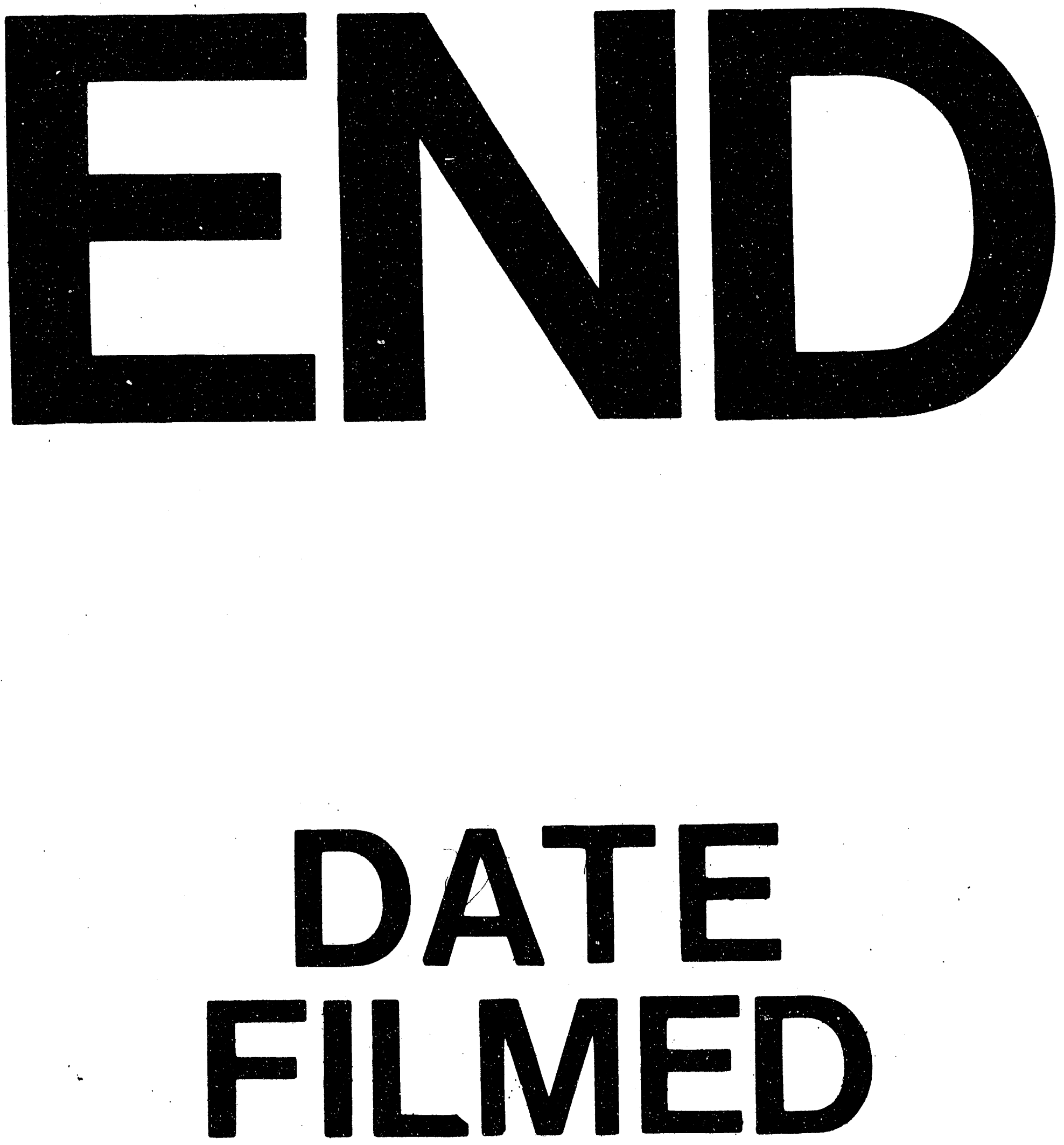

1

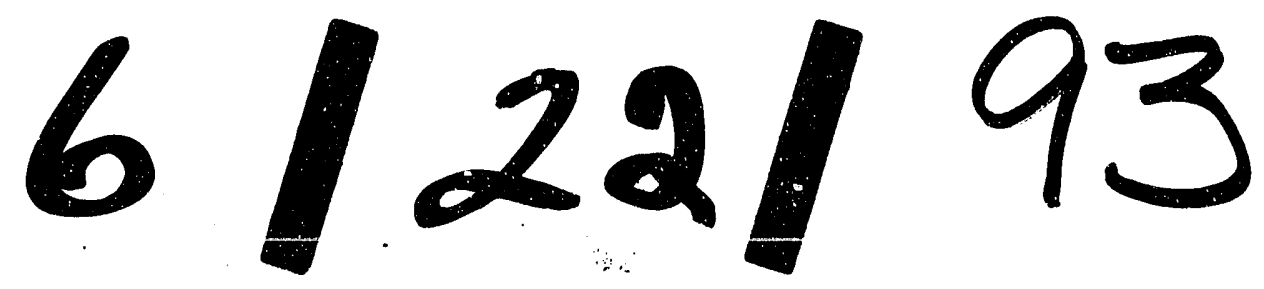


